Universidade de São Paulo

Instituto de Física

\title{
Termodinâmica de primeiros princípios aplicada a ligas de metais de transição.
}

\author{
Ney Sodre dos Santos
}

Orientadora: Profa. Dra. Helena Maria Petrilli

Tese apresentada ao Instituto
de Física para a obtenção do
título de Doutor em Ciências

\section{Comissão Examinadora:}

Profa. Dra. Helena Maria Petrilli (IF-USP)

Prof. Dr. Luis Gregório Dias da Silva (IF-USP)

Prof. Dr. João Francisco Justo Filho (POLI-USP)

Prof. Dr. Gilberto Carvalho Coelho (EEL-USP)

Prof. Dr. Leonardo Antonio Errico (UNLP-Ar)

São Paulo

2011 


\section{FICHA CATALOGRÁFICA \\ Preparada pelo Serviço de Biblioteca e Informação do Instituto de Física da Universidade de São Paulo}

Santos, Ney Sodré dos

Termodinâmica de primeiros princípios aplicada a ligas de metais de transição. - São Paulo, 2011.

Tese (Doutorado) - Universidade de São Paulo.

Instituto de Física, Departamento de Física dos Materiais e Mecânica.

Orientador: Profa. Dra. Helena Maria Petrilli

Área de Concentração: Física da Matéria Condensada

Unitermos: 1.Física da Matéria Condensada; 2. Física do Estado Sólido; 3. Cálculo de Estrutura Eletrônica;

4. Termodinâmica Computacional.

USP/IF/SBI-089/2011 
"Poichè: è dando, che si riceve;

Perdonando che

si è perdonati;

Morendo, che si

resuscita a Vita

Eterna”.

San Francesco 


\section{AGRADECIMENTOS}

A Deus sem O qual seria impossível chegar até aqui.

Gostaria de agradecer a todos que contribuíram de maneira relevante para minha formação e, particularmente, com o trabalho aqui apresentado.

Em especial À Professora Helena Maria Petrilli pela atenção, paciência, dedicação e contribuição para minha formação acadêmica e pessoal.

Aos amigos do grupo Nanomol, pelos ótimos momentos que passamos juntos.

Aos bons amigos de São Paulo: Joelson Cott Garcia, Rolando Larico, Ricardo Igarashi, Marcos Braun Gonçalves, Sandra Regina Rodrigues Ribeiro e Eliane Braga. Por causa de vocês São Paulo é um bom lugar para se estar. À todas as pessoas da minha Familia que souberam compreender que minha ausência teve por objetivo a realização de um sonho.

À CAPES, pelo apoio financeiro que permitiu a realização deste trabalho. Em última instância, ao bravo povo Brasileiro, que financiou a realização desta pesquisa.

Ao Departamento de Física dos Materiaise Mecânica (DFMT) do Instituto de Física da Universidade de São pelo suporte técnico em nossa jornada diária. 


\section{Resumo}

A termodinâmica computacional é uma ferramenta capaz de fornecer informações básicas sobre soluções e concentração de seus constituintes, em um dado sistema complexo a uma certa temperatura e pressão. Nos últimos anos, a união entre a teoria do funcional da densidade e a termodinâmica computacional tem renovado o estudo dos materiais intermetálicos ordenados, uma vez que os cálculos de estrutura eletrônica de primeiros princípios são hoje capazes de proporcionar resultados extremamente precisos para as energias de formação de compostos estequiométricos. Dentro deste contexto, investigamos os diagramas de fases dos sistemas Fe-Al, Mo$\mathrm{Fe}, \mathrm{Cr}-\mathrm{Al}, \mathrm{Fe}-\mathrm{Cr}, \mathrm{Fe}-\mathrm{Cr}-\mathrm{Al}$ na estrutura cúbica de corpo centrado utilizando o método "Full-Potential Linear Augmented Plane Waves"(FP-LAPW) aliado ao Método Variacional de "Clusters"(CVM) na aproximação do tetraedro irregular. Através do método FP-LAPW determinamos a energia total de configurações cristalinas dos sistemas cúbicos de corpo centrado. Esses valores são utilizados como parâmetros de entrada do CVM para a determinação do potencial termodinâmico do sistema em suas diferentes fases e os correspondentes equilíbrios entre essas fases em função da composição e da temperatura (diagrama de fases). Embora o Fe-Al tenha grande interesse tecnológico, o seu comportamento magnético é bastante complicado. A inclusão de $\mathrm{Cr}$ na liga de Fe-Al tem um alto interesse industrial na utilização desta ligas em altas temperaturas, mas o banco de dados referentes as caracteristicas estruturais e eletrônicas são escassos. Neste contexto de aplicações procuramos investigar as características estruturais e eletrônicas buscando correlacionar as informações experimentais com as obtidas via cálculo de estrutura eletrônica e o CVM. 


\begin{abstract}
Thermodynamics is a computational tool capable of providing information basics solutions and concentrations of constituents in a given complex system at a given temperature and pressure. In recent years, the union between the density functional theory and computational thermodynamics has renewed study of ordered intermetallic materials, since the electronic structure calculations from first principles are now able to provide extremely accurate results for the energies of formation of stoichiometric compounds. in this context, we investigate the phase diagrams of the systems $\mathrm{Fe}-\mathrm{Al}, \mathrm{Fe}-$ $\mathrm{Mo}, \mathrm{Cr}-\mathrm{Al}, \mathrm{Fe}-\mathrm{Cr}, \mathrm{Fe}-\mathrm{Cr}-\mathrm{Al}$ in the body-centered cubic structure using the method Full-Potential Linear Augmented Plane Waves (FP-LAPW) ally the clusters variational method(CVM) in approximation of the tetrahedron irregular. Through the FP-LAPW method determines the total energy of the crystal with configurations body centered cubic systems. These values are used as input parameters of the CVM for the determination of the thermodynamic potential of the system in its different phases and the corresponding equilibrium between these phases as a function of composition and temperature (phase diagram). Although the $\mathrm{Fe}-\mathrm{Al}$ has a great interest technology, its magnetic behavior is quite complicated. The inclusion of $\mathrm{Cr}$ in $\mathrm{Fe}-\mathrm{Al}$ alloy has a high industrial interest in using this alloy in high temperatures, but the database regarding the structural characteristics and electronics are scarce. In this context we seek to investigate the application structural and electronic characteristics correlate the information seeking experiments with those obtained via the electronic structure calculation and the CVM.
\end{abstract}




\section{Sumário}

1 Introdução 4

1.0.1 Metodologia . . . . . . . . . . . . . 4

1.1 Escolha dos sistemas estudados . . . . . . . . . . . . . . . 9

2 Metodologia I - Métodos De Primeiros Princípios 13

2.0.1 O esquema de Kohn-Sham . . . . . . . . . . . . . . . 13

2.1 Descrição do método FP-LAPW . . . . . . . . . . . . . . . 20

2.2 Representação da densidade de carga e do potencial . . . . . . 22

2.3 Solução da equação de Poisson . . . . . . . . . . . . . . . . . . . 22

2.3.1 A matriz Hamiltoniana . . . . . . . . . . . . . 23

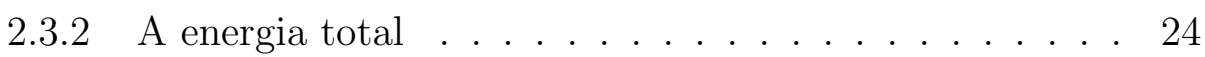

2.4 A autoconsistência no FP-LAPW . . . . . . . . . . 25

2.5 Implementação da base LAPW no código WIEN2K . . . . . . 27

3 Metodologia II - O Método Variacional de Clusters (CVM) 31

3.1 Definições . . . . . . . . . . . . . . . . . . 31

3.1.1 Espécies . . . . . . . . . . . . . . . . . . . 31

3.1 .2 Variáveis Configuracionais . . . . . . . . . . . . 31

3.1.3 Cluster . . . . . . . . . . . . . . . . . . 32

3.1.4 Probabilidade de Cluster . . . . . . . . . . . . 33

3.1.5 Probabilidade Configuracional . . . . . . . . . . 33

3.2 O CVM na aproximação do tetraedro irregular . . . . . . . . . 35

3.2.1 História do CVM . . . . . . . . . . . . . . 36

3.2.2 Ensemble microcanônico. . . . . . . . . . . . . . . . . 39

3.2.3 Configuração do tetraedro . . . . . . . . . . . . . . . 40

3.2.4 Entropia . . . . . . . . . . . . . . . . 45

3.2 .5 Energia Interna . . . . . . . . . . . . . . 46

3.2.6 Energia Livre . . . . . . . . . . . . . . . . . . 49

3.2.7 Minimização da energia livre a temperaturas finitas . . 50

3.2.8 Componentes com momento magnético . . . . . . . . 51 
4 Resultados e Discussões: 01 - Estruturas de Referência (Sistemas Unários)

4.1 Convergência na energia total . . . . . . . . . . . . . . 53

4.2 Escolha do termo de troca e correlação. . . . . . . . . . . . 56

5 Resultados e Discussões: 02 - As Influências das Premissas do Cálculo Ab-initio no Diagrama de fases $\quad 59$

5.1 Metodologia . . . . . . . . . . . . . . . . . 59

5.2 Resultados e discussões das premissas do cálculo . . . . . . . . 63

6 Resultados e Discussões: 03 - Sistemas Binários 68

6.1 Sistemas FeAl . . . . . . . . . . . . . . . . . . . 68

6.2 Sistemas $\mathrm{Fe}-\mathrm{Cr} \ldots \ldots \ldots$. . . . . . . . . . . . . . . . . . 79

6.3 Sistemas $\mathrm{Al}-\mathrm{Cr} \ldots \ldots$. . . . . . . . . . . . . . 87

7 Resultados e Discussões: 04- Sistema Ternário Fe-Cr-Al 95

7.1 Sistema Ternário Fe-Cr-Al . . . . . . . . . . . . . . . 95

8 Sumário 103

8.0.1 Parâmetros hiperfinos . . . . . . . . . . . . . . . 105 


\section{Capítulo 1}

\section{Introdução}

Exporemos neste trabalho a metodologia utilizada para obter os diagramas de fases na representação composição por temperatura. Logo após, indicaremos quais foram os fatores que nos motivaram na escolha dos sistemas estudados e faremos uma descrição dos trabalhos que consideramos mais relevantes sobre os sistemas estudados.

\subsubsection{Metodologia}

Diagrama de fases são representações gráficas entre duas ou mais variáveis termodinâmicas de estado que associam a cada ponto ordenado, ou seja, a cada estado do sistema a fase corresponde ao equilíbrio homogêneo ou fases correspondentes ao equilíbrio heterogêneo para este estado. Os diagramas de fases são de grande interesse prático na ciência dos materiais. Um método tradicional para a determinação de diagramas de fases envolve a elaboração de diversas ligas com composições diferentes e cobrindo toda a região que se deseja estudar no diagrama de fases. Amostras são retiradas de cada liga e tratadas a diferentes temperaturas, por um tempo suficiente, para que o equilíbrio seja estabelecido [1]. As microestruturas de cada amostra na temperatura de equilíbrio, ou seja, quais fases, com que composições e em que quantidades, estão presentes na amostra são usualmente investigadas, por exemplo, por têmpera ${ }^{1}$ e análise metalográfica de onde se obtém o diagrama de fases na representação concentração versus temperatura através da comparação entre as microestruturas obtidas para equilíbrios de composição e temperaturas similares. Esta metodologia foi utilizada, por exemplo, para se obter o diagrama de fases composição versus temperatura do sistema Ti-Fe

\footnotetext{
${ }^{1}$ consiste do resfriamento brusco em um meio como a água ou salmoura com o objetivo de se "congelar o estado de alta temperatura"
} 
[2]. Uma alternativa a este procedimento é utilizar outros métodos experimentais (difração de raios $\mathrm{X}$, difração em altas temperaturas, microscopia eletrônica de transmissão, etc.) combinados a modelos termodinâmicos, tais como o CVM (Cluster Variation Method), MC (Monte Carlo)[3], formalismo CALPHAD (Calculation of Phases Diagram) [4], etc. Isto é, utiliza-se resultados obtidos por métodos experimentais, tais como atividade química dos componentes, energia de formação em função da composição, temperatura crítica de ordenamento, etc., como dados de entrada para um formalismo teórico onde se obtém o respectivo diagrama de fases. Esta combinação é possível devido a chamada "autoconsistência" termodinâmica: Um modelo termodinâmico capaz de reproduzir o diagrama de fases do sistema deve automaticamente reproduzir outras propriedades termodinâmicas e vice-versa [5]. Esta metodologia recebe o nome de termodinâmica computacional, foi cunhada no Instituto Real de Tecnologia (KTH) de Estocolmo, Suécia na década de 90, no departamento de metalurgia física. Como exemplo, entre os inúmeros trabalhos, citaremos a título de ilustração duas publicações que utilizam esta metodologia.

- C. E. Dahmani et. al. estudaram o diagrama de fases do sistema Ni-Pt [6]. Foram utilizados difração de raio X e medidas in local ${ }^{2}$ da resistividade elétrica para a determinação das temperaturas de transformação das fases $L 1_{0}$ e $L 1_{2}$ na fase desordenada $F C C-A 1$. As temperaturas de Curie foram medidas para fases ordenadas e desordenadas. O método termodinâmico utilizado foi o CVM. Os autores indicam que o método da aproximação de Bragg-Williams (ABW) [7] e [8] não reproduz a transição de primeira ordem na estequiometria $\mathrm{AB}$. $\mathrm{O}$ resultado do CVM mostra uma boa concordância na topologia do diagrama com o observado experimentalmente. O máximo das linhas de separação das fases, os valores das temperaturas também estão em boa concordância com os valores obtidos experimentalmente. É importante ressaltar que esta concordância é consequência do ajuste dos parâmetros e, portanto, não é surpreendente.

- N. Dupin et. al. estudaram o diagrama de fases do sistema ternário AlCr-Ni [9]. Foi utilizado o formalismo CALPHAD para o cálculo do diagrama de fases. Neste trabalho são modeladas com uma única equação a fase desordenada BCC-A2 e a fase ordenada B2.

\footnotetext{
${ }^{2}$ Termo em latim que significa "no local", refere-se fundamentalmente a medidas feitas nas condições de solicitação da amostra para a qual ocorrem os fenômenos que estão sendo estudados. No presente caso refere-se a medidas de resistividade elétrica em altas temperaturas.
} 
O formalismo CALPHAD é frequentemente utilizado na indústria para fazer o diagrama de fases composição versus temperatura, estando incluído no formalismo geral da termodinâmica computacional. Uma grande porcentagem de diagramas de fases (binários e ternários) que existem na literatura foi feita utilizando esta metodologia, isto é, combinando resultados experimentais e modelos termodinâmicos incluídos no formalismo CALPHAD [10]. Note-se que, o termo CALPHAD é também utilizado como nome de uma revista de publicação seriada. O formalismo CALPHAD mistura uma grande quantidade de resultados experimentais, e os modelos ali incorporados utilizam uma grande quantidade de parâmetros para obter os diagramas de fases. Os modelos incorporados neste formalismo são baseados em expansões polinomiais de energia livre de excesso ${ }^{3}$ com diferentes graus de sofisticação. Estes modelos produzem algoritmos extremamente rápidos, necessários para o modelamento de processos envolvendo sistemas de interesse tecnológico, porém a sua simplicidade resulta em pouca consistência física. Por exemplo, mostra-se que determinadas equações ainda quando obedecem a relação de Gibbs-Duhem, estando incorretas no significado termodinâmico [11]. A introdução de modelos com um embasamento físico mais consistente no formalismo CALPHAD, foi proposta no artigo [12]. As justificativas para isso são: uma maior confiança nas extrapolações da energia livre molar de Gibbs de uma determinada fase para regiões do diagrama inacessíveis ao experimento, um maior grau de autoconsistência termodinâmica e principalmente uma maior compatibilidade com os métodos de primeiros princípios. Um destes modelos com maior embasamento físico é o CVM.

Paralelamente a estes desenvolvimentos os avanços obtidos nos métodos de Primeiros Princípios, permitiram determinar com grande precisão propriedades estruturais (parâmetro de rede, energia total, etc.) e eletrônica (densidade de estados, etc.) de um sistema qualquer partindo apenas do número de elétrons (Z) de cada componente desse sistema e suas respectivas posições no cristal. A continuação lógica deste desenvolvimento é a combinação dos dois procedimentos, a termodinâmica computacional e os métodos ab-initio, com os resultados dos últimos substituindo parcial ou totalmente os resultados experimentais requeridos para o primeiro. Através de uma aliança entre o modelo termodinâmico com os modelos ab-initio poderemos obter os diagramas de fases de um determinado sistema conhecendo apenas o número de elétrons de cada componente do sistema e sua respectiva posição no cristal. Esta metodologia foi denominada termodinâmica de primeiros princípios em uma revisão feita por De Fontaine [13]. No entanto, as atividades de pesquisa uti-

\footnotetext{
${ }^{3}$ Energia livre de excesso é a diferença entre a energia real do sistema e a energia de um sistema ideal.
} 
lizando esta metodologia são muito anteriores a esta data, é necessário cautela a respeito da nomenclatura utilizada. O desenvolvimento destas atividades de pesquisa é razoavelmente recente e alguns termos não estão consolidados na literatura, como é o caso do termo termodinâmica de primeiros princípios, já que diversos trabalhos que se encaixam na definição de De Fontaine não o mencionam. No presente trabalho adota-se integralmente a definição por este autor. A título de ilustração apresentaremos alguns trabalhos utilizando esta metodologia entre os inúmeros existente na literatura.

- M. Sluiter et al. estudaram o diagrama de fases do sistema Al-Li [14]. Foi utilizado o método FP-LAPW combinado com o formalismo CVM. O cálculo do equilíbrio metaestável no sistema Al-Li é interessante do ponto de vista tecnológico por causa da precipitação da fase $\mathrm{Al}_{3} \mathrm{Li}$ que é responsável pelo endurecimento de ligas ricas em alumínio. Ligas de Al-Li são de confecção extremamente difícil principalmente devido á alta reatividade com o oxigênio e à alta pressão de vapor do lítio que prejudicam a investigação experimental. Além disso, cálculos de primeiros princípios têm a vantagem de fornecer uma visão mais transparente das transições ordem-desordem observadas no sistema. Devemos ressaltar que a metodologia utilizada nesta publicação é a mesma utilizada no presente trabalho. O que nos diferencia, é que trabalhamos sistemas contendo o Fe como um elemento de nosso sistema o que permitirá analisar o efeito do magnetismo sobre o diagrama de fases.

- Mark Asta et al. estudaram o diagrama de fases composição versus temperatura do sistema Ti-Al baseado na estrutura FCC [15]. Foi utilizado o método de primeiros princípios Full-Potential Linear Muffin Tin Orbitals (FP-LMTO) com o CVM. Os compostos intermetálicos ordenados encontrados no sistema Ti-Al são particularmente promissores para aplicações aeroespaciais, porque além de possuírem alta resistência mecânica em alta temperatura, na concentração equiatômica (50\% de cada componente) são menos densos do que as super ligas á base de $\mathrm{Ni}$, usadas tradicionalmente para estas aplicações. O interesse em se desenvolver um estudo por termodinâmica de primeiros princípios neste caso é causado pela dificuldade de obtenção destas ligas. Isto ocorre devido à tendência de absorção de oxigênio e nitrogênio da atmosfera, o que tem forte influência sobre os equilíbrios entre as fases do sistema [16]. Um ano mais tarde, Mark Asta [17] apresenta novamente o diagrama de fases composição versus temperatura do mesmo sistema, Ti-Al, mas desta vez baseados nas estruturas FCC e HCP. 
- A. Pasturel et al. estudaram o diagrama de fases $\mathrm{Ni}-\mathrm{Al}$ baseados na estrutura FCC e BCC [18]. Foi utilizado o método Linear Muffin Tin Orbitals (LMTO) e o CVM. O sistema Ni-Al apresenta interesse tanto tecnológico quanto teórico. Os aluminetos de níquel apresentam propriedades interessantes tais como baixa densidade, elevado ponto de fusão e possuem elevada resistência em altas temperaturas. Além disso, a fase $L 2_{1}-N i_{3} A l$ é a base da liga comercial IC-221M que apresenta um incremento da resistência mecânica com o aumento da temperatura, ao contrário do que ocorre para a grande maioria dos materiais. Na parte teórica, no cálculo ab-initio, o estudo da densidade de estados (DOS), é também de interesse para determinar a contribuição da banda d do Ni, sobre o sistema Ni-Al. Tem-se evidência de uma forte hibridização entre a banda d do Ni e p do Al [19].

Entre os trabalhos que não usam o termo "termodinâmica de primeiros princípios", mas utilizam esta metodologia, podemos citar a título de ilustração:

- C. Colinet e A. Pasturel realizaram cálculos teóricos dos sistemas Cu$\mathrm{Ni}, \mathrm{Ag}-\mathrm{Ni}$ e Au-Ni baseados na estrutura FCC [20]. Estes autores utilizaram o método FP-LMTO juntamente com o CVM para calcular o domo de imiscibilidade ${ }^{4}$ dos sistemas $\mathrm{Cu}-\mathrm{Ni}$, $\mathrm{Au}-\mathrm{Ni}$ e $\mathrm{Ag}-\mathrm{Ni}$, com máximos aproximadamente nas temperaturas $900 \mathrm{~K}, 1200 \mathrm{~K}$ e 4000 $\mathrm{K}$ respectivamente. Neste trabalho, foi incrementada mais uma contribuição na energia total, pelo uso do modelo de Debye para calcular a energia livre vibracional.

- F. Lechermann e M. Fähnle estudaram o diagrama de fases do sistema Ni-Al baseado na estrutura BCC [21]. Foi utilizado o método ab-initio real space cluster expansion (CE) e o CVM. Neste trabalho mostrase que quando se considera a contribuição das vacâncias (lacunas, na terminologia metalúrgica) para a energia livre, o diagrama de fases é substancialmente modificado. Mostra-se também que a temperatura de transição B2/A2 prevista está na ordem de 8000 K. Neste trabalho os autores utilizam a denominação "Mecânica Estatística ab-initio" para este esquema.

Nestas brevíssimas citações de trabalhos, que correspondem à metodologia chamada "termodinâmica computacional" ou "termodinâmica de primeiros

\footnotetext{
${ }^{4}$ É uma região do diagrama de fases com a forma de domo dentro da qual não há miscibilidade total entre os componentes na mesma fase, isto é, coexistem duas fases com a mesma estrutura, mas com composições diferentes.
} 
princípios" pode-se notar que o assunto é objeto de pesquisas bastante intensa com aplicações tanto no domínio tecnológico quanto no acadêmico. Além disso, o procedimento está sendo constantemente aprimorado na tentativa de descrever sistemas cada vez mais complexos. No presente trabalho, utilizamos a "termodinâmica de primeiros princípios". Obtemos o diagrama de fases utilizando o método ab-initio FP-LAPW juntamente com o formalismo CVM. Escolhemos trabalhar com o FP-LAPW, porque é reconhecido na literatura como uma ferramenta eficiente para cálculos de estrutura eletrônica, especialmente para o cálculo da energia total de sistemas metálicos. A adoção do CVM para o presente trabalho se deve ao fato de ser este um método versátil, que permite calcular facilmente o potencial termodinâmico de sistemas com um número arbitrário de componentes, em oposição as simulações de Monte Carlo. Além do mais, o Método de Monte Carlo, por ser numericamente exato, tem um alto custo computacional para a obtenção de propriedades termodinâmicas. Por outro lado, o CVM por ser um método aproximado, requer um investimento computacional muito menor. Como na literatura as duas metodologias têm fornecido resultados semelhantes, por exemplo: $\mathrm{Cu}-\mathrm{Ag}$ [22], Fe-Al[23], Fe-Si, Fe-Co e V-Mn [24] assim como Gd [25], escolhemos trabalhar com o CVM. Nas últimas décadas, L. G. Ferreira et al., estudaram tanto o CVM como o Método Monte Carlo [26]. Note-se, entretanto, que na literatura também se encontram críticas com relação ao uso do CVM. Alguns autores o consideram muito "lento" para aplicações comerciais, que exigem a realização de diversos cálculos de equilíbrio em um curto espaço de tempo, advogando o uso do formalismo CALPHAD [27]. Outros autores já consideram o CVM como "muito simplificado", advogando o uso de algoritmos do Método de Monte Carlo no cálculo das propriedades termodinâmicas.

\subsection{Escolha dos sistemas estudados}

Os materiais intermetálicos ordenados, "utilizados para fins estruturais", e em particular os aluminetos de metais de transição têm atraido a atenção do circuito das engenharias nas últimas décadas devido a uma série de características únicas que os tornam particularmente atraentes para aplicações a temperaturas altas e moderadas [15]. Entre estas podemos citar:

1. Alta estabilidade estrutural devido às estruturas cristalinas complexas (em comparação às ligas metálicas desordenadas) e altas temperaturas de fusão; 
2. Boa resistência específica devido aos altos teores de alumínio, que reduzem a densidade do material;

3. De boa a excelente resistência à oxidação, à sulfetação devido à formação de camadas passivantes compostas de óxidos de alta entalpia de formação (nos aluminetos: $\mathrm{Al}_{2} \mathrm{O}_{3}$ ).

Segundo S. C. Deevi et al. "... aluminetos de Ni e Fe possuem concentrações suficientemente elevadas de $\mathrm{Al}$ (isto é, da ordem de $8 \%$ até $32 \%$ de peso) para formar uma camada de alumina contínua e aderente. Em contraste, quase $98 \%$ de ligas e superligas capazes de operar acima de $700{ }^{\circ} \mathrm{C}$ em ambientes oxidantes contém menos de $2 \%$ de peso do $\mathrm{Al}$ e invariantemente o $\mathrm{Cr}$ em concentrações ao redor de $18 \%$ de peso para promover resistência à oxidação. As ligas que contêm $\mathrm{Cr}$ formam $\mathrm{Cr}_{2} \mathrm{O}_{3}$ na exposição ao ar ou ao oxigênio e a dissociação de $\mathrm{Cr}_{2} \mathrm{O}_{3}$ para $\mathrm{CrO}_{3}$ limita sua resistência à oxidação até $950{ }^{\circ} C^{\prime \prime}$ [28]. A estas propriedades favoráveis, que tornam estes materiais possíveis concorrentes ao aços inoxidáveis para aplicações em altas temperaturas, contrapõem-se uma baixa ductibilidade ${ }^{5}$ e tenacidade $^{6}$ em temperatura ambiente. Entre os materiais intermetálicos ordenados, atualmente em desenvolvimento, podemos citar: as ligas baseadas no sistema Ti-Al [29] que apresentam densidades ainda mais reduzidas, mesmo em relação aos outros intermetálicos e as ligas baseadas no sistema Ni-Al, principalmente na forma da fase B2 (NiAl) que é estável em uma ampla faixa de composições e temperaturas [21]. Entretanto, tanto o Ni como o Ti são o que se costuma denominar de "matérias - primas estratégicas" para as quais há reservas minerais limitadas no mundo, tornando-se materiais de alto custo intrínseco [30]. Acrescente-se a isto o alto custo de produção destas ligas. Isto se deve ao fato de que o Ti-Al apresenta alta afinidade por nitrogênio e oxigênio, as altas temperaturas de processamento e o $\mathrm{Ni}-\mathrm{Al}$ apresenta elevada fragilidade a frio. A consequência disto é que estas ligas são adequadas apenas para a produção de componentes de alto valor agregado, tipicamente na indústria aeroespacial. Por outro lado o Fe é abundante na natureza, quer seja na forma de reservas minerais ou na forma de sucata, o que contribui para uma forte redução do custo intrínseco. Neste sentido, o sistema Fe-Al, abre uma "janela de mercados" na concorrência comercial aos aços inoxidáveis e com as "superligas" à base de níquel e de cobalto para uso em altas temperaturas [30]. Isto justifica o investimento em pesquisa e desenvolvimento

\footnotetext{
${ }^{5}$ Refere-se à capacidade do material se deformar plasticamente sem se fraturar, quando submetido a esforços de tração.

${ }^{6} \mathrm{E}$ uma medida da energia necessária para fraturar um corpo de prova padrão. Tenacidade a fratura se refere à resistência que o material oferece à propagação instável de uma trinca.
} 
deste material e o presente trabalho se insere neste esforço. Sabe-se que ligas do sistema Fe-Al tem boas propriedades mecânicas a quente ${ }^{7}$, que fazem delas candidatas para aplicações estruturais em ambiente hostil (por exemplo, atmosferas oxidantes) e em temperaturas intermediárias [31]. Exemplos de propriedades são:

1. Baixa densidade, já que a densidade do $\mathrm{Al}$ é $2.70 \mathrm{~g} / \mathrm{cm}^{3}$ e a do Fe é $7.86 \mathrm{~g} / \mathrm{cm}^{3}$ [32]; ligas baseadas na fase B2-FeAl apresentam densidades ao redor de $6 \mathrm{~g} / \mathrm{cm}^{3}$ [30];

2. Alta resistência mecânica a temperaturas elevadas (aproximadamente até $800 \mathrm{~K})$ [31];

3. Boa resistente a oxidação [31];

4. Baixo custo de fabricação [30].

O Fe-Al, além das propriedades de suas ligas mencionadas acima, é considerado um sistema protótipo para estudar as reações de ordenação atômica (configuracional) de ligas do tipo BCC, uma vez que esta é a estrutura estável sobre uma ampla faixa de composição que vai desde o Fe puro até quase 50 at.\% $\mathrm{Al}$ [33]. As interações de ordem magnética também são importantes neste sistema. Por exemplo, existe uma controvérsia na literatura entre medidas experimentais e resultados teóricos sobre o Fe-Al (B2) apresentar ou não magnetismo. Este será um dos aspectos abordados durante a análise dos resultados deste sistema. Entretanto, o sistema Fe-Al tem baixa ductilidade e tenacidade na temperatura ambiente [15], o que nos motiva a investigar um possível aprimoramento neste aspecto deste material. Existe na literatura uma grande quantidade de trabalhos, enfocando tanto o ponto de vista acadêmico como os tecnológicos direcionados a entender os fenômenos envolvidos na deformação plástica do sistema Fe-Al, com o objetivo de contornar esta fragilidade a frio. Alguns indícios sugerem que a transição entre o comportamento dúctil Nas últimas décadas, L. G. Ferreira et al., estudaram tanto o CVM como o Método Monte Carlo [26]. Note-se, entretanto, que na literatura também se encontram críticas com relação ao uso do CVM. Alguns autores o consideram muito "lento" para aplicações comerciais, que exigem a realização de diversos cálculos de equilíbrio em um curto espaço de tempo, advogando o uso do formalismo CALPHAD [27]. Outros autores já consideram

\footnotetext{
${ }^{7}$ Propriedades mecânicas a quente se referem as propriedades medidas acima de temperaturas onde os fenômenos de difusão passam a ser notados, tipicamente da ordem de 5-\% da temperatura de fusão (absoluta) do material. Para ligas Fe-Al nas composições aqui estudadas estas temperaturas devem ser superiores a aproximadamente à $1000 \mathrm{~K}$.
} 
o CVM como "muito simplificado", advogando o uso de algoritmos do Método de Monte Carlo no cálculo das propriedades termodinâmicas. 


\section{Capítulo 2}

\section{Metodologia I - Métodos De Primeiros Princípios}

Neste capítulo mencionaremos inicialmente as aproximações que são feitas para que a equação de Schrödinger (que envolve muitos corpos em um sólido e que conduz na maioria dos casos a uma inviabilidade da sua solução) se converta em uma expressão tratável computacionalmente conhecida como conjunto de equações de Kohn-Sham (KS). Este esquema é bastante conhecido na literatura e já foi tratado exaustivamente em diversos textos [34, 35]. Por este motivo, nos limitaremos a discorrer brevemente sobre o assunto. Posteriormente faremos uma descrição do método de primeiros princípios utilizado neste trabalho, o Full - Potential Linearized - Augmented - Plane - Waves (FP-LAPW), que resolve as equações de KS para os compostos estudados. O método FP-LAPW também é bastante conhecido na literatura e sendo assim não é nosso objetivo neste capítulo fazer uma descrição detalhada deste método, mas sim colocar os pontos mais importantes para uma visão geral da abordagem que utilizamos.

\subsubsection{O esquema de Kohn-Sham}

Sabe-se que as propriedades físicas da matéria são devidas as interações entre átomos ou moléculas e que os elétrons são responsáveis por uma série de fenômenos importantes na física do estado sólido. São estas interações que determinam se um sólido é condutor, semicondutor, ou isolante, além de serem responsáveis pelas propriedades de transporte. O cálculo da estrutura eletrônica de um material é importante no estudo de suas características físicas, assim como de seu comportamento diante de condições externas como pressão, temperatura, campos elétricos, etc.. Ao falar de estruturas eletrônica estamos nos referindo a distribuição dos estados eletrônicos em uma dada 
configuração espacial dos átomos. Para este tipo de cálculo temos atualmente uma grande variedade de métodos que têm como objetivo básico resolver a equação de Schrödinger independente do tempo[35].

$$
\hat{H} \Psi=E \Psi,
$$

onde o operador hamiltoniano $\hat{\Psi}$, para um sistema com m-elétrons e Nnúcleos é dado por[35],

$$
\hat{H}=\hat{T}_{e}+\hat{T}_{N}+\hat{V}_{N N}+\hat{V}_{N e}+\hat{V}_{e e} .
$$

Nesta expressão, $\hat{T}_{e}$ é o operador energia cinética dos elétrons, $\hat{T}_{N}$ é o operador energia cinética dos núcleos, $\hat{V}_{N e}$ é o operador energia potencial da interação dos elétrons com os núcleos e, finalmente $\hat{V}_{e e}$ é o operador energia potencial da interação dos elétrons. Estes operadores são definidos por:

$$
\begin{gathered}
\hat{T}_{e}=-\frac{\hbar^{2}}{2 m_{e}} \sum_{i}^{m} \nabla_{i}^{2} ; \nabla_{i}^{2}=\left(\frac{\partial^{2}}{\partial_{i}^{2}}+\right) \\
\hat{T}_{N}=-\frac{\hbar^{2}}{2} \sum_{\alpha}^{N} \frac{1}{M_{\alpha}} \nabla_{\alpha}^{2} ; \\
\hat{V}_{N N}=\sum_{\alpha, \beta}^{N} \sum_{\alpha<\beta}^{N} \frac{Z_{\alpha} Z_{\beta}}{\left|R_{\alpha}-R_{\beta}\right|} e^{2} ; \\
\hat{V}_{N e}=-\sum_{\alpha}^{N} \sum_{i}^{m} \frac{Z_{\alpha}}{\left|R_{\alpha}-r_{i}\right|} e^{2} ; \\
\hat{V}_{e e}=\sum_{i}^{m} \sum_{i<j}^{m} \frac{e^{2}}{\left|r_{i}-r_{j}\right|} .
\end{gathered}
$$

Aqui $\vec{r}$ e $\vec{R}$ denotam coordenadas eletrônicas e coordenadas nucleares, respectivamente e o laplaciano foi escrito, por simplicidade, em coordenadas cartesianas. Para sistemas de muitos corpos, a solução da equação de Schöredinger torna-se extremamente trabalhosa e, na maioria dos casos de interesse, é impossível obter soluções exatas devido ao enorme número de partículas envolvidas, como é o caso de materiais compostos por núcleos e elétrons interagentes. Uma maneira de contornar este problema é a utilização de algumas aproximações que descreveremos brevemente a seguir. A primeira aproximação consiste em desacoplar o movimento dos elétrons do 
movimento dos núcleos, esta aproximação é conhecida como "aproximação de Born-Oppenheimer" ou "aproximação adiabática"[56]. Devido à massa dos núcleos ser muito maior do que o elétron $\left(m / M \approx 10^{-4}\right.$ a $\left.10^{-5}\right)$, os elétrons deslocam-se muito mais rapidamente que os núcleos, justificando o uso desta aproximação. A função de onda total $\Psi(\vec{r}, \vec{R})$ que é a solução da equação de Schrödinger:

$$
\left\{\hat{T}_{e}+\hat{T}_{N}+\hat{V}_{N N}+\hat{V}_{N e}+\hat{V}_{e e}\right\} \Psi(\vec{r}, \vec{R}),
$$

pode ser expandida como:

$$
\Psi(\vec{r}, \vec{R})=\sum_{n} \Phi_{n}(\vec{R}) \psi_{n}(\vec{r}, \vec{R})
$$

Consideremos apenas a parte eletrônica da equação de Schrödinger[35]:

$$
\left\{\hat{T}_{e}+\hat{V}_{N N}+\hat{V}_{N e}+\hat{V}_{e e}\right\} \psi_{n}(\vec{r}, \vec{R})=\varepsilon_{n}(\vec{R}) \psi_{n}(\vec{r}, \vec{R}),
$$

onde não se considera o movimento dos núcleos, e portanto $\hat{T}_{N}$ é desprezado. Neste caso, a posição nuclear, $\vec{R}$, não é uma variável dinâmica e sim um parâmetro, e $\vec{r}$ é a variável coletiva da posição eletrônica $\vec{r}_{1}, \vec{r}_{2}, \vec{r}_{3}, \ldots$. A solução da parte eletrônica, equação (2.10), determina um conjunto completo de autovalores $\varepsilon_{n}(\vec{R})$ e autofunção $\psi_{n}(\vec{r}, \vec{R})$. Substituindo a equação $(2.9)$ em (2.8) e usando o resultado da parte eletrônica, teremos na aproximação adiabática[35]:

$$
\left\{\hat{T}_{N}+\varepsilon_{n}(R)\right\} \Phi_{n}(R)=E \Phi_{n}(R)
$$

A energia eletrônica total $\varepsilon_{n}(R)$, a qual também inclui a repulsão núcleo - núcleo, tem um papel de energia potencial. Desta forma o efeito resultante dos elétrons é acoplar os núcleos juntos, como uma mola, onde, para cada estado eletrônico, esta mola tem uma constante de força diferente. Consequentemente dentro da aproximação de Born-Oppenheimer as equações (2.10) nos dão informações sobre os estados eletrônicos do sistema e as equações (2.11) sobre o movimento nuclear. A segunda aproximação tem como objetivo transformar o problema de muitos corpos em um problema de um só corpo. Para isto, utilizaremos a teoria do funcional da densidade (Density Functional Theory-DFT) de Hohenberg-Kohn [36] no chamado esquema de Kohn e Sham - KS[37] que é constituída basicamente de dois teoremas válidos para um sistema de elétrons interagentes sujeitos a um potencial externo, $\nu_{\text {ext }}(\vec{r})$, que pode ser considerado como um potencial Colombiano devido aos núcleos. Em uma versão bem objetiva os teoremas podem ser escritos como: 
1. "A energia total do estado fundamental de um sistema eletrônico, sob a influência de um campo externo, é um funcional único de sua densidade eletrônica" [36, 38]

$$
E=E[\rho(\vec{r})]
$$

Note-se que, para um sistema com polarização de spin onde a densidade de carga é formada pela soma das densidades dos spins up ( $\uparrow)$ e down $(\downarrow)$, o funcional energia total será [38]

$$
E=E\left[\rho_{\uparrow}(\vec{r}), \rho_{\downarrow}(\vec{r})\right] .
$$

2. "Esse funcional energia total tem seu valor mínimo para a densidade correta do estado fundamental, levando-se em conta a conservação do número de partículas do sistema".

O funcional da energia total do estado fundamental pode ser escrito na forma,

$$
E_{T}[\rho]=V_{e x t}[\rho]+F[\rho] .
$$

Aqui $V_{e x t}[\rho]$ é também um funcional único da densidade eletrônica, expresso em termos do potencial $V_{\text {ext }}[\vec{r}]$ por

$$
V_{\text {ext }}[\rho]=\int \nu_{\text {ext }}(\vec{r}) \rho(\vec{r}) d \vec{r},
$$

e $F[\rho]$ é um funcional da densidade eletrônica, onde está incluída a energia cinética e os termos de interação elétron - elétron. $F[\rho]$ pode ser escrito como

$$
F[\rho]=T_{s}[\rho]+E_{c}[\rho]+E_{x c}[\rho],
$$

onde $T_{s}[\rho]$ denota o funcional energia cinética para um sistema de elétrons não interagentes, $E_{c}[\rho]$ é a energia devida a interação Colombiana entre os elétrons, chamada também de energia de Hartree, dada (em unidades de Hartree, $e^{2}=1$ ) por

$$
E_{c}[\rho]=\frac{1}{2} \iint \frac{\rho(\vec{r}) \rho\left(\vec{r}^{\prime}\right)}{\left|\vec{r}-\vec{r}^{\prime}\right|}
$$

e $E_{x c}[\rho]$ é, por definição, o funcional energia de troca - correlação (exchange - correlation) para o sistema formado por elétrons interagentes com densidade $\rho(\vec{r})$. No esquema de KS, em $E_{x c}[\rho]$, além de haver todos os efeitos de muitos corpos, há também uma contribuição devida a energia cinética dos elétrons, já que o termo $T_{s}[\rho]$ representa apenas a parte da energia cinética devida a um sistema de elétrons não interagentes. Na equação (2.16), o problema do desconhecimento da forma exata de $F[\rho]$ não foi resolvido, mas 
apenas redefinido, já que a forma exata do funcional $E_{x c}[\rho]$ também não é conhecida. Com as expressões acima, o funcional energia total fica [35]:

$$
E_{T}[\rho]=\int \nu_{e x t}(\vec{r}) \rho(\vec{r}) d \vec{r}+T_{s}[\rho]+\frac{1}{2} \int \frac{\rho(\vec{r}) \rho\left(\vec{r}^{\prime}\right)}{\left|\vec{r}-\vec{r}^{\prime}\right|} d \vec{r} d \vec{r}^{\prime}+E_{x c}[\rho]
$$

De acordo com o "princípio variacional", tomando a variação de $E(\rho)$, com o vínculo do número total de elétrons serem fixos, chega-se a [35]:

$$
\int \delta \rho(\vec{r})\left\{\frac{\delta T_{s}}{\delta \rho}+\nu_{\text {ext }}(\vec{r})+\int \frac{\rho(\vec{r})}{|\vec{r}-\vec{r}|} d \vec{r}+V_{x c}[\rho(\vec{r})]-\frac{\delta E[\rho]}{\delta \rho}\right\} d \vec{r}=0
$$

Aqui $V_{x c}[\rho(\vec{r})]$ é o potencial de troca-correlação, dado por

$$
V_{x c}[\rho(\vec{r})]=\frac{\partial E_{x c}[\rho(\vec{r})]}{\partial \rho(\vec{r})} .
$$

Ao se estudar um sistema de $\mathrm{m}$ - elétrons adota-se o formalismo de uma partícula no qual um sistema é descrito por orbitais $\varphi_{i}(\vec{r})$. Estes orbitais são preenchidos com dois elétrons cada (em um formalismo sem polarização de spin), em ordem crescente de suas energias, de tal forma que a densidade eletrônica pode ser escrita em termos de $\varphi_{i}$ e de seus complexos conjugados $\varphi_{i}^{*}$ como

$$
\rho(\vec{r})=\sum_{i}^{m}\left|\varphi_{i}(\vec{r})\right|^{2}=\sum_{i}^{m} \varphi_{i}^{*} \cdot \varphi_{i} .
$$

Substituindo a equação (2.21) na equação (2.19) chega-se ao seguinte conjunto de equações de uma partícula, expressa em unidades de Rydberg como:

$$
\left\{-\frac{1}{2} \nabla^{2}+V^{e f e}[\rho(\vec{r})]\right\} \varphi_{i}(\vec{r})=\varepsilon_{i} \varphi_{i}(\vec{r}), i=1, \ldots, m .
$$

Aqui $\varepsilon_{i}$ são as energias de uma partícula relacionadas com os orbitais $\varphi_{i}$ e $V^{e f e}[\rho(\vec{r})]$ é a energia potencial efetiva, dada por $[39,40]$

$$
V^{e f e}[\rho(\vec{r})]=V_{c}[\vec{r}, \rho(\vec{r})]+V_{x c}[\rho(\vec{r})]
$$

sendo que $V_{c}$ corresponde a energia potencial eletrostática chamada também de potencial de Coulomb, que contém a interação núcleo - elétron e a interação entre os elétrons, isto é [39, 40] 


$$
V^{e f e}(\vec{r})=\sum_{\alpha} \frac{-Z_{\alpha}}{\left|\vec{r}-\vec{R}_{\alpha}\right|}+\int \frac{\rho(\vec{r})}{|\vec{r}-\vec{r}|} d \vec{r}^{\prime}+V_{x c}[\rho(r)] .
$$

Nesta expressão, o primeiro termo refere-se ao potencial nuclear sobre o elétron em $\vec{r}, Z_{\alpha}$ é o número atômico do núcleo na posição $R_{\alpha}$, o segundo termo é o potencial eletrostático devido aos demais elétrons e finalmente temos o potencial de troca - correlação. É assim que a equação de Schrödinger (com resolução inviável na grande maioria dos casos, pois representa uma equação para $n$ partículas) se converte em um conjunto de $n$ equações, equações (2.22), para uma só partícula (com resolução viável), conhecido como equações de KS. Para que as equações de KS sejam resolvidas, autoconsistentemente, é necessário conhecer o termo $V_{x c}$, equação (2.20). Para isto adota-se alguma forma explicita para $E_{x c}[\rho]$. Note-se que se conhecêssemos exatamente $E_{x c}$ e $V_{x c}$, todos os efeitos de muitos corpos estariam em princípio incluídos. Entretanto na prática isto não é possível, porém existem várias aproximações para este termo [35, 41]. Neste trabalho apresentaremos duas aproximações mais utilizadas para o termo de troca - correlação, $E_{x c}[\rho]$. A primeira é a aproximação da densidade local (Local Density Approximation - LDA) com suas diversas parametrizações e extensões para o caso com polarização de spin onde é chamada de LSDA (Local Spin Density Approximation). Alguns exemplos são: Hedin - Lundqvist [42]; Moruzzi, Janak e Williams [43] e Perdew e Wang [44]. Na LDA, assume-se que a variação da densidade de carga, $\rho(\vec{r})$, é suficientemente lenta e o termo $E_{x}[\rho]$ é dado por [35]:

$$
E_{x c}[\rho]=E_{x c}^{L D A}=\int \rho(\vec{r}) \varepsilon_{x c}[\rho(\vec{r})] d \vec{r}
$$

Aqui o termo $\varepsilon_{x c}(\rho)$ é a energia de troca - correlação e correspondente á energia, por elétron, de um gás de elétrons interagentes com densidade de carga uniforme $\rho(\vec{r})$.

A segunda é a aproximação de gradiente generalizada (Generalized Gradient Approximation - GGA). Alguns exemplos são: PW86 [45]; B88 [46]; EV93 [47] e PBE96 [48]. Nesta aproximação, $E_{x c}$ é função da densidade de carga local e também do gradiente da densidade, ou seja:

$$
E_{x c}[\rho]=E_{x c}^{G G A}[\rho]=\int \rho(\vec{r}) \varepsilon_{x c}[\rho(\vec{r})] d \vec{r} .
$$

O objetivo é fazer uma segunda descrição, com a informação adicional contida no gradiente da densidade de carga.

Para calcular a estrutura eletrônica de um sistema resolvendo as equações de KS é necessário escolher uma aproximação para o $E_{x c}[\rho]$ (LDA ou GGA no 
caso). Escolhida uma aproximação, as equações de KS são resolvidas em um processo iterativo ou autoconsistente, o qual descrevemos a seguir: Primeiramente, escolhemos uma densidade inicial $\rho^{0}$ que vai fornecer o potencial efetivo, equação (2.24), para o sistema estudado. Este potencial, inserido nas equações de KS, (2.22), fornece os estados $\varphi_{i}$, que por sua vez devem produzir uma nova densidade de carga $\rho^{1}$ utilizando a equação (2.21). Comparamos as densidades de cargas inicial $\rho^{0}$ e final $\rho^{1}$. Se elas diferirem apenas dentro de um erro pré - estabelecido inicialmente, dizemos que o sistema está "convergido". Caso contrário, utiliza-se $\rho^{1}$ no lugar de $\rho^{0}$ para o novo processo iterativo, até atingir a convergência. Este procedimento geral cumpre-se para qualquer método que desenvolva as equações de KS auto - consistentemente. Entretanto existem diferentes maneiras de implementar este processo segundo a conveniência e adequação para cada tipo de problema. As equações de KS, para o caso de um cristal período podem ser reescritas como:

$$
\left[\hat{T}+\hat{V}^{e f e}(\vec{r})\right] \varphi_{n \vec{k}}(\vec{r})=\varepsilon_{n \vec{k}}(\vec{r}) .
$$

Aqui $\varphi_{n \vec{k}}(\vec{r})$ são as funções de onda de Bloch [32] referentes a um estado de vetor de onda $\vec{k}$ que pertence a banda $n$ e $\varepsilon_{n \vec{k}}$ são os autovalores que fornecem as bandas de energia do sistema estudado. Aqui por simplicidade descreveremos somente o tratamento não relativístico. Para obter a energia total, dentro da teoria do funcional da densidade, expressaremos o funcional energia cinética, $T_{s}[\rho]$, a partir das equações de KS. Para isto multiplicamos por $\varphi_{i}^{*}$ a equação $(2.27)$, onde $i=n \vec{k}$, fazendo depois a integração:

$$
\int \varphi_{i}^{*} \hat{T} \varphi_{i} d \tau+\int \varphi_{i}^{*} \hat{V}^{e f e} \varphi_{i} d \tau=\varepsilon_{i} \int \varphi_{i}^{*} \varphi_{i} d \tau
$$

Por construção (condição de ortonormalidade) a integral do lado direito é igual a unidade e do lado esquerdo $\hat{T}$ e $\hat{V}_{\text {efe }}$ são operadores. Em seguida realiza-se a somatória sobre todos os estados ocupados, $\sum_{i}$, obtendo-se:

$$
\sum_{i} \int \varphi_{i}^{*} \hat{T} \varphi_{i} d \tau+\sum_{i} \int \varphi_{i}^{*} \hat{V}^{e f e} \varphi_{i} d \tau=\sum_{i} \varepsilon_{i}
$$

o primeiro termo do lado esquerdo fornece a energia cinética em função da densidade, $T_{s}[\rho]$ e o segundo termo também se expressa em termos da densidade, equação (2.23). Desta forma a energia cinética fica,

$$
T[\rho]=\sum_{i} \varepsilon_{i}-\int V^{e f e}(\vec{r}) \rho(\vec{r}) d \vec{r} .
$$


Substituindo a equação acima, (2.30), na equação (2.18) obteremos a energia total, $E_{T}$. Descreveremos nas próximas seções o método FP-LAPW que foi adotado no presente trabalho.

\subsection{Descrição do método FP-LAPW}

O método FP-LAPW desenvolve autoconsistentemente as equações de KS, no esquema descrito na seção anterior e como será detalhado na seção 2.4. O FP-LAPW se desenvolve na mesma linha do método APW (Augmented Plane Wave), proposto por J. C. Slater [50] onde o espaço cristalino é dividido em duas regiões e diferentes expansões de base são usadas nessas regiões (Fig.2.1).

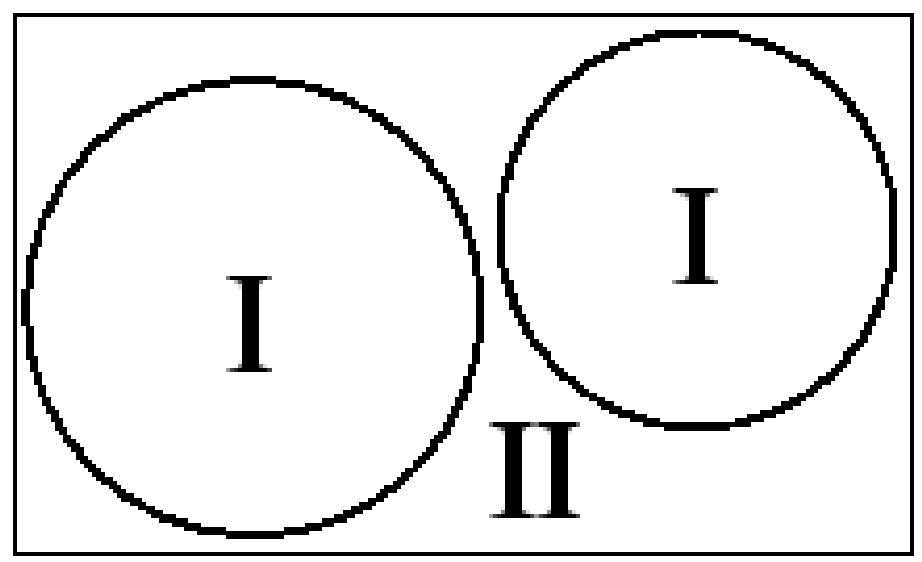

Figura 2.1: Divisão do espaço no método FP-LAPW. Figura retirada do manual do código Wien2k.

Denotamos por $I$, as regiões que são esferas de raio $\mathrm{R}$ não sobrepostas e centradas nas posições atômicas, e por $I I$, a outra por ser a região intersticial entre as esferas. Dentro das esferas a base é construída por uma combinação linear de funções radial vezes harmônicos esféricos $Y_{l m}(\vec{r})$, onde $l$ e $m$ são números quânticos orbital e magnético, respectivamente. No LAPW as funções de base são combinações lineares de uma função radial $u_{\nu l}\left(E_{\nu, r}\right)$, para cada valor de $l$, e sua derivada com relação á energia, $\dot{u}_{\nu l}\left(E_{\nu}, r\right)$, avaliada em uma energia fixa $E_{\nu}$, normalmente escolhida no centro da banda correspondente, $v_{\nu l}$ é a solução da equação radial [51, 38]:

$$
\left\{-\frac{d^{2}}{d r^{2}}+\frac{l(l+1)}{r^{2}}+V(r)-E_{\nu}\right\} \dot{r} u_{\nu l}(r)=0
$$


onde $V(r)$ é a componente esférica do potencial cristalino dentro da esfera. A função $\dot{u}_{\nu l}\left(E_{\nu}, r\right)$ satisfaz uma equação semelhante, porém não homogênea. Na região intersticial (II), onde o potencial é aproximadamente constante, a expansão da função de onda é obtida através de ondas planas, $\exp (i \vec{K} \vec{r})$, onde:

$$
\vec{K}=\vec{k}+\vec{G}
$$

sendo que $\vec{G}$ é um vetor de translação da rede recíproca e $\vec{k}$ um vetor de onda restrito a parte irredutível da primeira zona de Brillouin. As funções de base do LAPW, $\phi_{k}(\vec{r})$, são definidas então como[38, 40]:

$\phi_{\vec{K}}(\vec{r})= \begin{cases}\frac{1}{\Omega^{\frac{1}{2}}} \exp (i \vec{K} \vec{r}), & \text { para } r \in I I ; \\ \sum_{l, m}\left\{A_{l, m}(\vec{K}) u_{l}\left(E_{\nu}, r\right)+B_{l, m}(\vec{K}) \dot{u}_{l}\left(E_{\nu}, r\right)\right\} Y_{l, m}(\vec{r}), & \text { para } r \in I .\end{cases}$

Estas funções são usadas para a expansão das autofunções de KS [40] na forma:

$$
\psi_{\vec{k}, i}(\vec{r})=\sum_{|\vec{K}|<K^{w f}} C_{i}(\vec{K}) \phi_{\vec{K}}(\vec{r})
$$

Na equação (2.34), o "corte das funções de onda", $K^{w f}$, limita o número destes vetores e consequentemente a dimensão da base. Na equação (2.33), $\Omega$ é o volume da célula, $A_{l, m}(\vec{K})$ e $B_{l, m}(\vec{K})$ são coeficientes de expansão que são obtidos quando se impõem: a continuidade da função na fronteira da esfera, $r=R$, e a continuidade da derivada da função em $r=R$. Neste tratamento as funções dentro e fora da esfera são resolvidas separadamente, mas devem ser "casadas" na superfície da esfera para garantir a continuidade da densidade de carga. O fato de incluir a derivada da função radial, em relação à energia, na expansão da função de onda na região da esfera juntamente com a utilização do parâmetro $E_{\nu}$, no método LAPW, introduz um erro da ordem de $\left(\varepsilon-E_{\nu}\right)^{2}$ na função de onda, o qual leva a um erro de $\left(\varepsilon-E_{\nu}\right)^{4}$ na energia da banda. É assim que as "LAPWs" formam uma boa base em um razoável intervalo de energia, permitindo, na maioria dos casos e particularmente em metais, tratar a banda de valência com um único conjunto de $E_{\nu}$ e obter as bandas de energia para um determinado ponto $\vec{k}$ da zona de Brillouin. Além disto, podem-se introduzir também os chamados "orbitais locais"[38] para melhorar a base. 


\subsection{Representação da densidade de carga e do potencial}

A eficiência da base LAPW está na escolha das funções de onda nas duas regiões: expansões em harmônicos esféricos dentro das esferas e expansões em ondas planas na região intersticial. Variações rápidas das funções de onda implicam em uma rápida variação da densidade de carga e do potencial, Entretanto, a utilização direta desta representação resulta em um grande número de coeficientes a serem determinados. Uma maneira de reduzir este número é usar expansões que levem em conta a simetria do sistema. No LAPW, continuamos o esquema dual, a expansão na região intersticial é feita em "estrelas" e dentro dos átomos não equivalentes em "harmônicos de rede". As estrelas, $\Phi_{s}(\vec{r})$, são definidas como [38]:

$$
\Phi_{s}=\frac{1}{N_{o p}} \sum_{R} \cdot e^{i R G(r-t R)},
$$

aqui $R$ são os componentes rotacionais das operações do grupo espacial. As harmônicas de rede são definidas por [38]:

$$
K_{\nu, \alpha}\left(r-R_{\alpha}\right)=\sum_{m} C_{\nu, m}^{\alpha} Y_{l, m}\left(r-R_{\alpha}\right),
$$

sendo que $R_{\alpha}$ a posição do centro do átomo $\alpha$. Assim, a densidade de carga é dada por [38]:

$$
\phi(\vec{r})= \begin{cases}\sum_{s} a_{s} \Phi_{s}(\vec{r}), & \text { para } \vec{r} \in I I ; \\ \sum_{\nu} \rho_{\nu(\vec{r})} K_{\nu}(\vec{r}), & \text { para } \vec{r} \in I .\end{cases}
$$

Coerente com o estabelecido para a expansão da função de onda e para a densidade, o potencial do sistema é descrito por [40]:

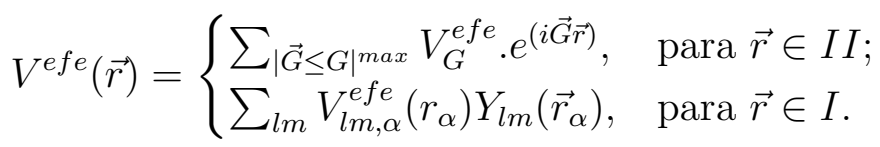

Assim nenhuma aproximação de forma é introduzida. A qualidade desta descrição do potencial total (full-potential:FP) é controlada pelo parâmetro de corte $G^{\text {max }}$ para os vetores da rede recíproca $\vec{G}$.

\subsection{Solução da equação de Poisson}

O potencial efetivo usado nas equações de KS contém o potencial de troca-correlação e o potencial de Coulomb, $V_{c}(\vec{r})$, equação $(2.23)$. O $v_{C}(\vec{r})$ 
é determinado pela densidade de carga (eletrônica mais nuclear) através da equação de Poisson (em unidades atômicas, $e^{2}=1$ ) $[38,52]$ :

$$
\nabla^{2} V_{c}(\vec{r})=-4 \pi \rho(\vec{r}),
$$

e pode ser escrita como uma serie de Fourrier [52]:

$$
V_{c}(\vec{r})=\sum_{G} V_{c} \cdot e^{(i \vec{G} \vec{r})}
$$

com $[38,52]$ :

$$
V_{c}(\vec{G})=\frac{4 \pi \rho(\vec{G})}{|\vec{G}|^{2}} .
$$

Dadas as condições de contorno, a integração da equação (2.39) pode ser executada eficientemente em uma pequena região. Entretanto, em geral a solução no espaço real não é uma solução facilmente obtida. A equação de Poisson no espaço recíproco, equação (2.41), tem solução em princípio trivial [38]. Infelizmente, no método LAPW, há uma rápida variação da densidade de carga dentro das esferas resultando no fato de que a expansão em série de Fourrier, $\rho(G)$ não seja convergente. Para solucionar este problema, D. R. Hamann [53] e E. Wimmer et. al. [54] desenvolveram um método baseado em potenciais de multipolos com o "problema de Dirichlet" para uma esfera [55]. O esquema consiste em substituir a verdadeira densidade de carga dentro das esferas por uma pseudo-carga, que tenha os mesmos momentos de multipolos e que na sua representação de Fourrier possua uma rápida convergência. Esta pseudo-carga é utilizada para obter o potencial de Coulomb correto no espaço todo, ou seja, tanto na região intersticial como dentro das esferas. O potencial dentro das esferas é obtido resolvendo-se o problema de contorno usando a densidade de carga verdadeira da região.

\subsubsection{A matriz Hamiltoniana}

Os elementos da matriz de sobreposição, $S_{\vec{G} \vec{G}^{\prime}}$, e os elementos da matriz hamiltoniana, $H_{\vec{G} \vec{G}^{\prime}}$, estão definidos por [38]:

$$
\begin{gathered}
S_{\vec{G} \vec{G}^{\prime}}=<\Phi_{G} \mid \Phi_{G^{\prime}}> \\
\mathrm{e} \\
H_{\vec{G} \vec{G}^{\prime}}=<\Phi_{G}|H| \Phi_{G^{\prime}}>.
\end{gathered}
$$


Estas equações podem ser decompostas em uma parte intersticial e em uma parte dentro da esfera. No caso, da hamiltoniana, a parte dentro da esfera pode ainda ser dividida em parte esférica (energia cinética mais a parte do potencial para $l=0$ ) e parte não esférica. Com isto, pode-se escrever a matriz de sobreposição, como [38]:

$$
S_{\vec{G} \vec{G}^{\prime}}=\frac{1}{\Omega} \int_{\Omega} d \vec{r} \cdot e^{i\left(\vec{G}^{\prime}-\vec{G}\right) \cdot \vec{r}} \Theta(\vec{r})+\sum_{\alpha} S_{\alpha}\left(\vec{G}, \vec{G}^{\prime}\right) .
$$

aqui $S_{\alpha}\left(\vec{G}, \vec{G}^{\prime}\right)$ é a contribuição da esfera $\alpha$ para o termo de sobreposição, $\Theta(\vec{r})$ é a função degrau, definida como zero dentro da esfera e um na região intersticial. A matriz hamiltoniana pode ser escrita como:

$$
\begin{aligned}
H_{\vec{G}, \vec{G}^{\prime}} & =\frac{1}{\Omega} \int_{\Omega} d \vec{r} \Theta(\vec{r}) e^{-i(\vec{G}+\vec{k}) \cdot \vec{r}}\left\{T+V_{P W}\right\} \cdot e^{i(\vec{G}+\vec{k}) \cdot \vec{r}} \\
& +\sum_{\alpha}\left\{H_{\alpha}\left(\vec{G}, \vec{G}^{\prime}\right)+V_{\alpha}^{N S}\left(\vec{G}, \vec{G}^{\prime}\right)\right\}
\end{aligned}
$$

onde $T$ é o operador energia cinética, $V_{P W}$ a parte do potencial na região intersticial, $H_{\alpha}\left(\vec{G}, \vec{G}^{\prime}\right)$ é a contribuição esférica para a hamiltoniana e $V_{\alpha}^{N S}\left(\vec{G}, \vec{G}^{\prime}\right)$ a contribuição não esférica, $l \neq 0$, do potencial na esfera $\alpha$.

\subsubsection{A energia total}

A energia total é uma importante grandeza física a ser calculada neste método e através de sua determinação podemos obter outras propriedades tais como parâmetro de rede, compressibilidade, etc. Note-se que no formalismo FP-LAPW não está incluída a temperatura, o que quer dizer que nossos cálculos são realizados para o estado fundamental, onde a temperatura é $T=0 K$. Utilizamos a equação (2.30) substituindo-se a equação (2.38) que descreve o potencial total na expressão da energia total, no FP-LAPW. Notese que esta é uma característica importante deste tipo de abordagem (FP), o que permite obter a energia total com uma boa precisão. Assim, a energia total adquire, no FP-LAPW, a forma [39]:

$$
E_{\text {Total }}[\rho]=\sum_{i} \varepsilon_{i}-\int_{\Omega} d \vec{r} . \rho(\vec{r})\left[\frac{1}{2} V_{c}\left(\vec{r}+V_{x c}(\vec{r})\right)\right]-\frac{1}{2} \sum_{\alpha} Z_{\alpha} V_{M}\left(\overrightarrow{R_{\alpha}}\right)+E_{x c} .
$$

aqui $\left[V_{M}\left(\vec{R}_{\alpha}\right)\right]$ é o potencial de Coulomb, em $\vec{R}_{\alpha}$, devido a todas as cargas no cristal a exceção da carga nuclear neste sítio, $Z_{\alpha}$, [39]: 


$$
V_{M}\left(\vec{R}_{\alpha}\right)=\int \frac{\rho(\vec{r}) \cdot d \vec{r}}{\left|\vec{r}-\vec{R}_{\alpha}\right|}-\sum_{\beta} \frac{Z_{\beta}}{\left|\vec{R}_{\beta}-\vec{R}_{\alpha}\right|} .
$$

Finalmente, é interessante lembrar que no caso de sólidos a energia será a soma da energia eletrônica mais a energia vibracional. Sabe-se no entanto que a energia eletrônica é, na maioria dos casos, muito maior que a energia vibracional o que nos permite resolver as equações de KS só para a parte eletrônica. No presente trabalho nos limitaremos ao tratamento da parte eletrônica não abordando as contribuições vibracionais.

\subsection{A autoconsistência no FP-LAPW}

Na descrição da autoconsistência que faremos nesta seção, os nomes em maiúscula, que estão entre parênteses, são os utilizados no código computacional WIEN2K e que são mostrados na figura 2.2.

Por ser um método de primeiro princípios (dentro da teoria do funcional da densidade) o FP-LAPW requer unicamente como dados de entrada o número de elétrons e as posições de cada componente a ser estudado. Com estes dados geramos nossa célula unitária. Nela o espaço é dividido em esferas sendo que, por exemplo, para o sistema $F e_{3} A l$, serão três esferas para o $F e$ e uma para o $A l$, cada uma centrada no correspondente sítio da rede. $\mathrm{O}$ primeiro passo é a verificação de que as esferas não se sobrepõem (NN). Isto dependerá de uma conveniente escolha dos raios das esferas.

Geralmente escolhe-se como raio a metade da distância dos primeiros vizinhos. Com esta escolha as esferas vão estar tangentes, tendo muito pouco espaço intersticial, o que nos leva a utilizar uma menor quantidade de ondas planas. Mas, se pretendemos fazer uma otimização do parâmetro de rede, o que significa ter de comprimir o sistema, pode acontecer uma sobreposição das esferas com esta escolha de raio. Para que isto não aconteça, o raio da esfera tem que ser menor que a metade da distância dos primeiros vizinhos. Note-se entretanto que, em princípio, respeitando o fato de que não haja sobreposição o resultado deve independer da escolha do raio, embora o custo computacional envolvido varie bastante. O passo seguinte é realizar um cálculo tipo atômico (embora confinado ao raio da esfera)(LSTART) para obter a energia total e a densidade eletrônica de cada átomo que compõem o sistema.

Aqui definimos como serão tratados no cálculo os diferentes orbitais, se como caroço ou valência. Em seguida são geradas as operações de simetria (SYMMETRY), assim como também os harmônicos de rede e as matrizes de rotação locais. Posteriormente geram-se os pontos $\vec{k}(\mathrm{KGEN})$ na parte 
irredutível da primeira zona de Brillouin. Até aqui, geramos as condições para que se obtenha a densidade eletrônica inicial, $\rho^{0}$, do cristal, o que é feito sobrepondo as densidades eletrônicas de cada átomo (DSTART). Com a densidade eletrônica inicial calculamos o potencial total (LAPW0), fazendo a soma do potencial de Coulomb e o potencial de troca-correlação. Com este potencial construímos os elementos da matriz hamiltoniana, que depois de ser diagonalizada (resolução da equação de KS) nos proporciona os autovalores e autovetores (LAPW1), ou seja, obtemos os auto-estados de uma partícula $\psi_{i}$. É assim que conseguimos as densidades eletrônicas dos estados de valência em cada ponto $\vec{k}$ (LAPW2) através da soma sobre todos os estados ocupados calculados até a energia de Fermi. Os estados de caroço (LCORE) são obtidos em um cálculo atômico, porém na presença do potencial do sólido. Para obter a nova densidade eletrônica, fazemos a soma entre os estados de caroço e valência. Comparamos então as densidades de entrada e saída do processo. Caso elas difiram dentro de um critério pré- estabelecido nosso sistema estará convergido. Caso contrário faz-se uma mistura para estabilizar a convergência, entre as densidades de entrada e de saída, utilizando um fator de mistura $Q$ :

$$
\rho_{\text {mistura }}(r)=(1-Q) \cdot \rho^{\text {entrada }}(r)+Q \cdot \rho^{\text {saida }}(r)
$$

Com a densidade eletrônica misturada reiniciamos o processo interativo até atingir a autoconsistência conforme o esquema da Figura 2.2.

Neste trabalho utilizamos a densidade de carga como indicador de critério de convergência. No código WIEN2K, além da densidade de carga pode-se utilizar a energia total ou a força como critério de convergência. Os parâmetros que definem a base ou procedimentos numéricos e podem ser variados na obtenção da convergência, são:

- LMAX-Este parâmetro nos define a quantidade de ondas parciais que serão usadas dentro da esfera.

- RKMAX- Este parâmetro nos define, de forma indireta, a quantidade de ondas planas utilizadas, segundo:

$$
R K M A X=\min \left(R_{\alpha}\right) \max (|\vec{G}|)
$$

onde $R_{\alpha}$ é o raio da esfera $\alpha$ e $\vec{G}$ percorre os vetores da rede recíproca.

- O número de pontos $\vec{k}$ - Número de pontos na parte irredutível da primeira zona de Brillouin, no espaço recíproco. 


\subsection{Implementação da base LAPW no código WIEN2K}

O método LAPW, descrito nas seções anteriores, é implementado em vários pacotes computacionais. Um dos mais elaborados e usados é o código WIEN2k. Esse pacote é constituído de vários programas independentes que são interligados. Na seção anterior abordamos de forma breve a aplicação destes programas durante o cálculo LAPW. A seguir são dadas as principais tarefas de cada um dos programas que o constitui, desde os que fazem parte da inicialização de um cálculo (NN, SGROUP, SYMMETRY, LSTART, KGEN e DSTART) aos que correspondem à parte do ciclo autoconsistente (LAPW0, LAPW1, LAPW2, LCORE e MIXER). Para executar um cálculo autoconsistente usando esse código é preciso executar, antes, uma série de pequenos programas auxiliares que usam um arquivo com as informações sobre o material, os quais gerarão a partir delas dados de entrada para os principais programas a serem executados no ciclo autoconsistente. No início do cálculo é necessário um arquivo de entrada contendo as informações sobre os parâmetros de rede, as posições e espécie atômica que compõem o sólido. Nesse método, nenhuma informação a respeito das propriedades já conhecidas do material é introduzida nos cálculos. Métodos com essas características são denominados de primeiros princípios ou ab-initio. Assim, após preparar o arquivo de entrada, cuidadosamente, o processo inicial é rodar, na seqüência:

- $\mathrm{NN}$

Esse programa calcula a distância dos vizinhos de todos os átomos e verifica se houve superposição das esferas atômicas. O programa, além disso, permite verificar se os átomos de um mesmo tipo foram especificados corretamente no arquivo de entrada.

- SGROUP

Determina o grupo espacial da estrutura definida no arquivo de entrada e todos os elementos de simetria dos grupos pontuais dos sítios não equivalentes.

- SYMMETRY

Gera as operações de simetria do grupo espacial e calcula valores de 1 e m para expansão da densidade eletrônica.

- LSTART

Para executar um cálculo autoconsistente usando esse código é preciso executar, antes, uma série de pequenos programas auxiliares que usam um arquivo com as informações a cerca do material, os quais gerarão 
a partir delas dados de entrada para os principais programas a serem executados no ciclo autoconsistente. Calcula a densidade eletrônica dos átomos livres (constituintes do composto estudado) que será usada no programa DSTART, e determina como os diferentes orbitais serão tratados. Para isso, o programa interage pedindo para especificar uma energia de corte, isto é, um valor de energia que separará os elétrons que serão tratados como sendo da valência, e os que serão tratados como de caroço (elétrons em orbitais cujas energias são inferiores ao valor de corte e estão completamente dentro da esfera atômica). Os dados de entrada que são gerados a partir da execução desses programas, surgem como valores padrões, os quais devem ser analisados cuidadosamente para cada sistema estudado. O LSTART, por exemplo, cria um arquivo que contém vários parâmetros, os quais dizem respeito à convergência do cálculo e devem, portanto, ser verificados. Um dos parâmetros mais importantes é o Rmt X Kmax, pois é ele quem define o tamanho da base e, portanto, a precisão dos cálculos. Esse parâmetro corresponde usualmente a valores entre 5 e 9 (quando se usa a base APW+lo) ou entre 6 e 10 (para a base LAPW). O termo Rmt corresponde ao raio da menor esfera muffin-tin da célula cristalina e o Kmax é o raio de corte no espaço recíproco. Todos os vetores da rede recíproca que estiverem contidos na esfera com raio Kmax serão contados no conjunto base. Outro parâmetro que deve ser conferido é o Lmax, isto é, o máximo valor de $\mathrm{L}$ que determina a quantidade de harmônicos esféricos permitidos para as funções atômicas dentro das esferas muffin-tin.

\section{- KGEN}

Gera a rede de vetores de onda k na parte irredutível da zona de Brillouin. Ao executar esse programa ele, interativamente, pedirá o número de pontos k que serão usados no cálculo. Esse número é particular para cada sistema.

\section{- DSTART}

Esse programa gera uma densidade eletrônica cristalina inicial por superposição das densidades eletrônicas dos átomos constituintes, calculadas no LSTART.

Após executados esses programas é feita a analise dos dados gerados, o ciclo autoconsistente para a solução das equações de Kohn e Sham, pode ser, finalmente, rodado. O ciclo é repetido até atingir o critério de convergência especificado pelo usuário. O ciclo autoconsistente executa os seguintes programas: 


\section{- LAPW0}

Esse programa usa a densidade calculada pelo DSTART como densidade inicial para construir o potencial efetivo, e calcula o potencial total como soma do potencial coulombiano e o potencial de troca e correlação.

\section{- LAPW1}

Monta o hamiltoniano e as matrizes de overlap, e encontra por diagonalização dessas os autovalores e autovetores. O processo de diagonalização corresponde à parte do cálculo que consome maior tempo.

\section{- LAPW2}

Calcula a energia de Fermi e determina a nova densidade de carga eletrônica no cristal, fazendo integração para todos os estados ocupados e para todos os pontos k na primeira zona de Brillouin.

\section{- LCORE}

Determina os autovalores da parte esférica do potencial e as correspondentes densidades de cargas dos elétrons de caroço, bem como ajusta os níveis energéticos dos elétrons no caroço.

- MIXER Esse programa mistura as densidades eletrônicas de caroço e valência para produzirem uma nova densidade eletrônica do cristal a ser utilizada na próxima interação, até que o critério de convergência não seja alcançado. Ver na figura (2.2), o fluxo dos programas descritos anteriormente.

Uma vez convergidos os cálculos, temos à nossa disposição a completa descrição quântica, no estado fundamental, do sistema em estudo. Em outras palavras, obtiveram-se as autofunções e autovalores do hamiltoniano de Kohn-Sham, e a partir desses pode-se calcular várias propriedades como densidade dos estados (DOS), estrutura das bandas e propriedades ópticas, as quais não serão descritas neste texto devido ao foco do trabalho. 


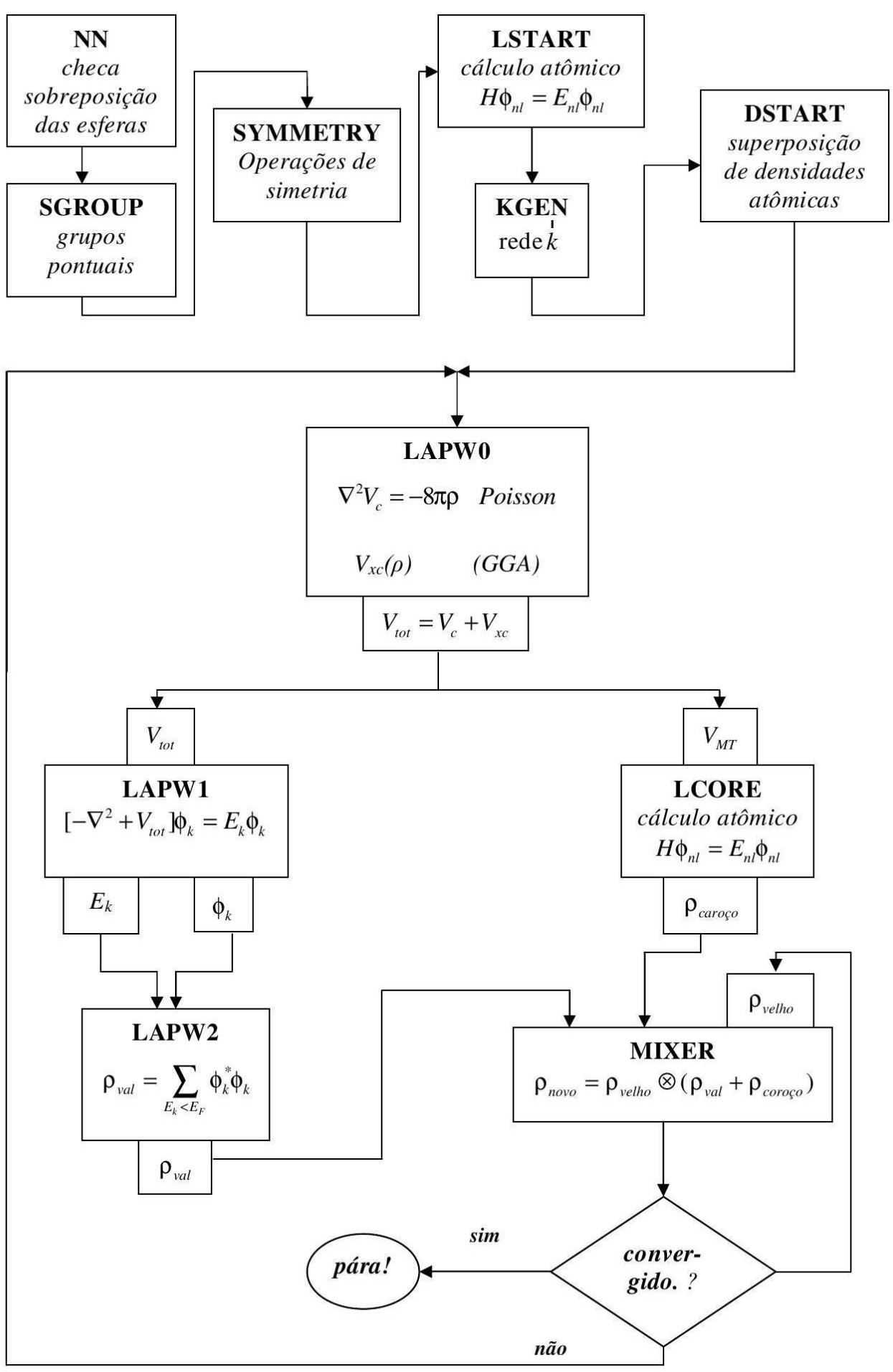

Figura 2.2: Fluxograma dos programas principais que fazem parte do código WIEN2K. 


\section{Capítulo 3}

\section{Metodologia II - O Método Variacional de Clusters (CVM)}

Neste capítulo apresentamos o Método Variacional de Clusters (CVM). Primeiramente serão apresentados alguns conceitos básicos, para uma melhor compreensão do método. Posteriormente a termodinâmica de sistemas multicomponentes será discutida para finalmente descrevermos propriamente o CVM.

\subsection{Definições}

\subsubsection{Espécies}

Por espécies entende-se uma classe de átomos que se distinguem dos outros por alguma característica física peculiar. Exemplos de espécies são os elementos químicos, elementos químicos em diferentes projeções de spin no eixo "z" ou íons com diferentes graus de ionização.

\subsubsection{Variáveis Configuracionais}

Consideremos um sistema cristalino com $\mathrm{N}$ sítios e n espécies $i$ ( $i=A, B$, $C, \ldots)$ sendo $\mathrm{N}$ a soma sobre todos os átomos do sistema, $N=\sum_{i} N_{i}$ [59]. Por exemplo, para um composto binário do tipo $\mathrm{Fe}_{3} \mathrm{Al}$, teremos duas espécies (n=2) onde $A=F e$ e $B=A l$, alternativamente para um composto do tipo $F e_{2} A l M o$ teremos três espécies $(\mathrm{n}=3)$ onde $A=F e, B=A l$ e $C=M o$. A distribuição das espécies atômicas sobre os sítios da rede define uma configuração. Para especificar a configuração precisamos de um operador que identifique as espécies atômicas sobre um sítio $\mathbf{n}$ qualquer da 
rede. Neste sentido, escolhemos o operador $\sigma_{n}$ que associa números inteiros identificando as diferentes espécies atômicas que ocupam o sítio $\alpha$, isto é, $\sigma_{n}=1,2,3, \ldots, n$. Notem que embora existam outras escolhas esta é a mais frequentemente utilizada. Assim uma configuração do cristal especificada pelo vetor $\vec{\sigma}=\left(\sigma_{1}, \sigma_{2}, \sigma_{3}, \ldots, \sigma_{N}\right)$. O número total de configurações possíveis é $n^{N}$ [59]. Por exemplo, para um sistema binário $A B$, sem momento magnético, $\sigma_{n}=1,2$ isto significa que no sítio $n$ teremos o número 1 quando este for ocupado por um $\mathrm{Fe}$ e o número 2 quando for ocupado por um $\mathrm{Al}$. Analogamente, a posição 1 da rede pode ser ocupada por um $F e$ ou um $A l$, isto é, $\sigma_{1}=F e$ ou $\sigma_{1}=A l\left(\sigma_{1}=1\right.$ ou $\left.\sigma_{1}=2\right)$. Duas configurações diferentes são por exemplo:

$$
\begin{aligned}
& \text { - } \vec{\sigma}=(F e, A l, F e, \ldots, A l) \text { ou } \vec{\sigma}=(1,2,1, \ldots, 2) \\
& \text { - } \vec{\sigma}=(F e, F e, A l, \ldots, F e) \text { ou } \vec{\sigma}=(1,1,2, \ldots, 1) .
\end{aligned}
$$

Para um composto binário $A B$, com momento magnético presente no átomo $A$ (no caso das espécies serem definidas por: $A \uparrow$ e $A \downarrow$ ), teremos $\sigma_{\alpha}=1,2,3(A \uparrow=1, A \downarrow=2$ e $B=3)$. O operador $\sigma_{\alpha}$ é chamado também de variável de spin devido a correspondência formal com o modelo de Ising. Qualquer função de $\sigma_{\alpha}$, incluindo o próprio $\sigma_{\alpha}$, é chamado de "variável configuracional"'[59].

\subsubsection{Cluster}

Para um sistema cristalino com N muito grande é impossível contar com todas as configurações diferentes. A natureza estatística do problema, entretanto, garante que apenas um subconjunto pequeno e altamente degenerado (são as configurações que têm a mesma energia) destas configurações influencia as propriedades do sistema. Desta forma, uma solução aproximada pode ser obtida considerando-se apenas as configurações pertencentes a este subconjunto. Uma estratégia para se escolher este subconjunto é a utilização de uma configuração correspondente a uma unidade muito pequena chamada cluster. Definimos um cluster como um conjunto de pontos da rede: $\alpha_{1}, \alpha_{2}, \alpha_{3}, \ldots, \alpha_{r}$ com $\mathrm{r} \ll \mathrm{N}$. Uma configuração sobre este cluster é dada por: $\left(\sigma_{1}, \sigma_{2}, \sigma_{3}, \ldots, \sigma_{r}\right)$. Desse modo em um cluster de $r$ sítios tem-se $n^{r}$ configurações diferentes, sendo este número muito menor do que $n^{N}$. Um subconjunto de um cluster também é um cluster. Para diferenciá-lo do cluster escolhido para representar o sistema iremos designá-lo por sub-cluster; exemplos de sub-clusters são os pontos: $\sigma_{1}$, o par $\sigma_{1} \sigma_{2}$, o tripleto $\sigma_{1} \sigma_{2} \sigma_{3}$ e assim por diante. 


\subsubsection{Probabilidade de Cluster}

O sistema completo, com $\mathrm{N}$ sítios é substituido por outro composto por $M_{r}$ clusters, onde cada cluster atua como uma entidade estatisticamente independente dos outros. Definimos $M_{\sigma_{r}}$ como o número de clusters com uma dada configuração $\sigma_{r}$ no sistema. Ao invés de $M\left(\vec{\sigma}_{r}\right)$, é comum utilizar $\rho$, dado por:

$$
\rho\left(\vec{\sigma}_{r}\right)=\frac{M\left(\vec{\sigma}_{r}\right)}{M_{r}}
$$

onde $M_{r}$ é o número de clusters de tamanho r contidos no sistema com N-sítios. As probabilidades de clusters especificam a configuração na aproximação de clusters com r-sítios e constituem a mais importante variável configuracional do sistema.

\subsubsection{Probabilidade Configuracional}

As funções termodinâmica de um sistema vão depender da probabilidade do cluster escolhido e de todas as probabilidades dos sub-clusters contidos neste cluster de r-sítios que descreve uma configuração, $\nu_{t o t}$, dado por [59]

$$
\nu_{t o t}=\left(\begin{array}{l}
r \\
1
\end{array}\right) K+\left(\begin{array}{l}
r \\
2
\end{array}\right) K^{2}+\ldots+\left(\begin{array}{l}
r \\
r
\end{array}\right) K^{r}=(K+1)^{R}-1 .
$$

Aqui para um ponto há $\mathrm{K}$ escolhas de ser ocupado por um elemento e $\left(\begin{array}{l}r \\ 1\end{array}\right)$ combinações ${ }^{1}$ de um ponto no cluster, para um par temos $K^{2}$ escolhas de dois elementos e $\left(\begin{array}{l}r \\ 2\end{array}\right)$ combinações de um para de pontos neste cluster, e assim por diante. Para a equação (3.2) foi utilizada a expansão binomial:

$$
(K+1)^{r}=\left(\begin{array}{l}
r \\
0
\end{array}\right) K^{0}+\left(\begin{array}{l}
r \\
1
\end{array}\right) K^{1}+\ldots+\left(\begin{array}{l}
r \\
r
\end{array}\right) K^{r} .
$$

Entretanto, as contribuições parciais para a $\nu_{\text {tot }}$ (probabilidades configuracionais de ponto, dupla,etc.) não são todas independentes. Se as probabilidades de um cluster são dadas, então as probabilidades de todos os subclusters podem ser obtidas por somatórias parciais. Por exemplo, para um sub-cluster de 3-pontos temos [59]

$$
\rho\left(\sigma_{1}, \sigma_{2}, \sigma_{3}\right)=\sum_{\sigma_{4}, \ldots, \sigma_{r}} \rho\left(\sigma 1, \sigma_{2}, \sigma_{3}, \sigma_{4}, \ldots, \sigma_{r}\right)
$$

\footnotetext{
${ }^{1}$ Lembrando que as combinações possíveis de $\mathrm{x}$ objetos idênticos em n posições é: $\left(\begin{array}{l}n \\ x\end{array}\right)=$ $\frac{n !}{x !(n-x) !}$
} 
onde os índices das somatórias percorrem todos os possíveis valores de $\sigma_{4}, \ldots, \sigma_{r}$. O número de somatórias parciais, $\nu_{p}$, é definido como

$$
G=H-T S .
$$

O estado de equilíbrio de um sistema a temperatura e pressão constantes é definido por um mínimo de $\mathrm{G}$.

- Energia livre de Gibbs molar $\left(G_{m}\right)$ - Define-se a energia livre de Gibbs molar como $G_{m}=G / N$ em unidades de $\mathrm{J} / \mathrm{mol}$.

- Energia livre de Gibbs molar parcial - Para um sistema qualquer pode-se definir a energia livre de Gibbs molar pela relação:

$$
G=\sum_{i=1}^{K} N_{i}\left(\frac{\partial G}{\partial N_{i}}\right)_{T, P, N_{j}, i \neq j} .
$$

A quantidade entre parênteses recebe o nome de energia livre de Gibbs molar parcial da espécie $i\left(\bar{G}_{m}^{i}\right)$. Por exemplo, a energia livre de Gibbs molar parcial de uma solução ideal será dada por:

$$
\bar{G}_{m}^{i}=\bar{G}_{0 m}^{i}+R T \ln \left(x_{i}\right),
$$

onde R é a constante dos gases, $\bar{G}_{0 m}^{i}$ é a energia livre de Gibbs molar padrão definida para o estado de referência, no caso em que $\mathrm{P}=1 \mathrm{~atm}$. e $x_{i}$ é a fração molar da espécie $i$. Por definição temos:

$$
\mu_{i}=\left(\frac{\partial G}{\partial N_{i}}\right)^{T, P, N_{j}, i \neq j}
$$

Desta forma obtemos uma das identidades fundamentais da termodinâmica, também conhecida como potencial químico, $\mu_{i}$ :

$$
\mu_{i}=\bar{G}_{m}^{i}
$$

- Energia Livre de Gibbs molar parcial de uma solução qualquer. - Para uma solução qualquer teremos por definição:

$$
\bar{G}_{m}^{i}=\bar{G}_{0 m}^{i}+R T \ln \left(a_{i}\right),
$$

onde $a_{i}$ é chamada de atividade da espécie $i$. Por comparação com 3.7 podemos definir o coeficiente de atividade $\left(\gamma_{i}\right)$ :

$$
\bar{G}_{m}^{i}=\bar{G}_{0 m}^{i}+R T \ln \left(x_{i}\right)+R T \ln \left(\gamma_{i}\right) .
$$

De onde temos que

$$
a_{i}=\gamma_{i} x_{i}
$$


- Energia livre de mistura. - Substituindo a expressão (3.11) em (3.6), podemos escrever:

$$
G=\sum_{i=1}^{K} \bar{G}_{0 m}^{i}+R T \sum_{i=1}^{K} N_{i} \ln \left(a_{i}\right) .
$$

O primeiro termo do lado direito da equação acima é idêntico á energia livre de um sistema hipotético composto pelas $\mathrm{K}$ espécies do sistema caso não houvesse interação entre elas. O segundo termo contém, portanto, toda a informação sobre a interação entre as espécies. Por definição denominamos estas quantidades por: Energia livre de Gibbs de referência $\left(G_{0}^{r e f}\right)$ e energia livre de Gibbs de mistura $\left(\Delta G^{m i x}\right)$. Desde que o mesmo estado de referência seja escolhido para cada espécie do sistema, o termo de referência não irá influir no equilíbrio entre as fases. Os cálculos CVM apresentados no presente trabalho usam todos o mesmo estado de referência para todas as espécies (fase BCC, na temperatura do cálculo) e, portanto, apenas o termo de mistura precisa ser considerado no cálculo dos diagramas de fases.

\subsection{O CVM na aproximação do tetraedro ir- regular}

Seguiremos nesta seção o mesmo desenvolvimento realizado nas referências [60] e [1]. Quando se estuda a termodinâmica de sistemas multicomponentes, é natural utilizarmos como variáveis a pressão $(\mathrm{P})$, o volume $(\mathrm{V})$, a temperatura $(\mathrm{T})$, o número total de átomos $(\mathrm{N})$ (constante) e o conjunto das frações molares dos compostos $\left(x_{i}\right)$ Um estado de equilíbrio é então definido pelo mínimo da energia livre de Gibbs, dado por:

$$
G\left(N, T, P,\left(x_{i}\right)\right)=U\left(N, T, P,\left(x_{i}\right)\right)-T S\left(N, T, P,\left(x_{i}\right)\right)+P V\left(N, T, P,\left(x_{i}\right)\right),
$$

sendo $U$ a energia interna definida por;

$$
U\left(N, T, P,\left(x_{i}\right)\right)=T S\left(N, T, P,\left(x_{i}\right)\right)-P V\left(N, T, P,\left(x_{i}\right)\right)+\sum_{i}^{K} \mu_{i} x_{i},
$$

onde $K$ é o número de espécies e $\mu_{i}$ o potencial químico da espécie $i$, definido na equação (3.8). Em sistemas condensados o termo $P V$ freqüentemente é desconsiderado[1] em primeira aproximação, nas equações (3.14) e (3.15), tal procedimento será utilizado pois a variação do volume é usualmente pequena em comparação com as demais grandezas físicas, tendo assim 
pouca influência no mínimo de $G$. A versão do algoritmo CVM utilizado no presente trabalho, implementado por Cláudio Geraldo Schön e Luiz Tadeu Fernandes Eleno, desconsidera este termo. A dependência da energia livre de Gibbs (G) com o número de átomos de cada espécie (o qual é expressada através de $\left(x_{i}\right)$ ), não é a mais adequada para o uso prático nos cálculos de diagramas de fases. Isto devido ao fato que usualmente o sistema pode ser encontrado em um estado heterogêneo, isto é, um estado onde duas ou mais fases coexistem em equilíbrio, para determinados valores de temperatura $(T)$ e concentração $\left(x_{i}\right)$. Entretanto, o que se mantém constante em todas as fases é o potencial químico $\left(\mu_{i}\right)$ de cada espécie. Para uma dada temperatura, o que definirá o equilíbrio entre duas fases $\varphi$ e $\xi$ será o equilíbrio do potencial químico entre estas fases, tal equilíbrio é definido pela relação:

$$
\left.\mu_{i}\right|_{\varphi}=\left.\mu_{i}\right|_{\xi} \forall i
$$

Para um sistema binário A-B, teríamos:

$$
\mu_{A}^{\varphi}=\mu_{A}^{\xi}, \mu_{B}^{\varphi}=\mu_{B}^{\xi} .
$$

É conveniente expressar a equação (3.14) em função de $\left(\mu_{i}\right)$ ao invés de $\left(x_{i}\right)$. isto pode ser obtido aplicando-se a correspondente transformada de Legendre sobre a energia livre de Gibbs $(G)$, como mostrado em [61]. O equilíbrio do sistema neste caso será dado pelo máximo do Potencial GranCanônico, $\Omega_{G P}\left(N, T,\left(\mu_{i}\right)\right)$, que pode ser obtido com o conhecimento da função das frações molares dos componentes $\left(x_{i}\right)$. A determinação desta função, entretanto, não é uma tarefa trivial e portanto o uso do potencial gran-canônico $\left(\Omega_{G P}\right)$ para a solução do problema é pouco prático. Um método alternativo para a minimização da energia livre de Gibbs $(G)$ sob a restrição do potencial químico $\left(\mu_{i}\right)$ é empregado na literatura e será descrito a seguir.

\subsubsection{História do CVM}

Em 1951, R.Kikuchi [67] propôs um novo método, a idéia básica do método conforme o artigo original (ainda não denominada Cluster Variational Method-CVM ${ }^{2}$ ), é introduzir uma figura geométrica no sistema cristalino e contar todas as possíveis configurações considerando as interações entre os átomos dessa figura escolhida. Posteriormente essa figura recebeu o nome de cluster. Neste sentido, a primeira coisa a fazer no CVM é definir um cluster básico. Neste artigo pioneiro Kikuchi introduziu uma expressão geral para a entropia de sistemas cristalinos em função das probabilidades de ocupação de pontos, pares, triângulos, etc., até um número pré-estabelecido de

\footnotetext{
${ }^{2}$ Em português, método de variação de agregados.
} 
sítios no sistema cristalino, por isto o método é aproximado. Esta é uma das principais características do CVM, a união entre a análise combinatória e a geometria tridimensional, que geralmente fornece aproximações para a entropia obtidas de modo muito mais fácil que os métodos anteriormente propostos. O CVM pode ser encarado como uma hierarquia de aproximações, como bem salientou Kikuchi em 1986 ao revisar o seu trabalho [68]. Cada aproximação considera interações restritas a um determinado número fixo de átomos, formando uma figura geométrica (um cluster) indeformável no reticulado. Começando com o ponto como um cluster básico, o CVM equivale à aproximação de Bragg-Williams (ou Currie-Weiss para a transição ferromagnética/paramagnética); com o par de primeiros vizinhos, o CVM fornece equações idênticas às do modelo de Bethe; com o quadrado, ao modelo de Kramers-Wannier; e assim por diante. O modelo original de Kikuchi para a obtenção da entropia configuracional, no entanto, é muito difícil de ser utilizado como um formalismo geral. Para o caso do tetraedro irregular (IT) no reticulado BCC, por exemplo, a dedução da expressão da entropia seguindo o método original de Kikuchi nunca foi publicada. Ainda assim, o processo é facilmente generalizado, conforme observou Baker em 1953 [69]. A partir de então diversos métodos diferentes foram criados para a determinação da entropia configuracional[ $70,71,72,73,74,78,75,79,77,76,80,81,82,83]$. No CVM, as propriedades termodinâmicas do sistema escolhido para o modelamento são ajustadas através de alguns parâmetros, ditos parâmetros de interação do CVM relativos a este sistema. Os parâmetros de interação do CVM estão relacionados às energias de formação das superestruturas a serem modeladas [84]. Dentre as abordagens utilizadas para o ajuste destes parâmetrosque não são necessariamente únicos- podemos ressaltar três de maior importância:

- ajuste a dados experimentais de diagramas de fases [66];

- ajuste as propriedades termodinâmicas, tais como dados de atividade e temperatura críticas de ordenamento [85];

- cálculos de primeiro princípios (ou ab-initio), com base em determinações de estrutura eletrônica [86].

A experiência tem mostrado que os ajustes através de dados experimentais conseguem prever satisfatoriamente a escala de temperatura do diagrama de fases experimental, mas não reproduz de forma satisfatória a energia de formação experimental destes supereticulados. Fornecendo valores cerca de 30 a $50 \%$ menores. Por outro lado, métodos de primeiro princípios são capazes de fornecer valores muito precisos da energia de formação (a 0K) dos 
supereticulados (com erro menor que $10 \%$ do valor experimental). Mesmo assim, cálculos utilizando estes dados fornecem na maioria dos casos uma escala de temperaturas para o diagrama de fases duas a três vezes maiores que a experimental.

No presente trabalho, utilizamos como cluster básico o tetraedro irregular, por ser mais simples, aplicado ao reticulado BCC, seguindo a notação desenvolvida na seção 3.1.3, este sistema é definido pelo vetor $\bar{\sigma}=$ $\left(\sigma_{1}, \sigma_{2}, \sigma_{3}, \sigma_{4}\right)$ ou ainda podemos chamar esta configuração da seguinte forma $\bar{\sigma}=\left(\sigma_{\alpha}, \sigma_{\beta}, \sigma_{\gamma}, \sigma_{\delta}\right)$. Entretanto, é conveniente utilizar uma notação diferente, que será enunciada ao longo do texto. Embora, consideremos inicialmente os sistemas binários e depois os sistemas ternários, as equações utilizadas podem ser aplicadas para um sistema de multicomponentes sem nenhuma modificação nos códigos[87].

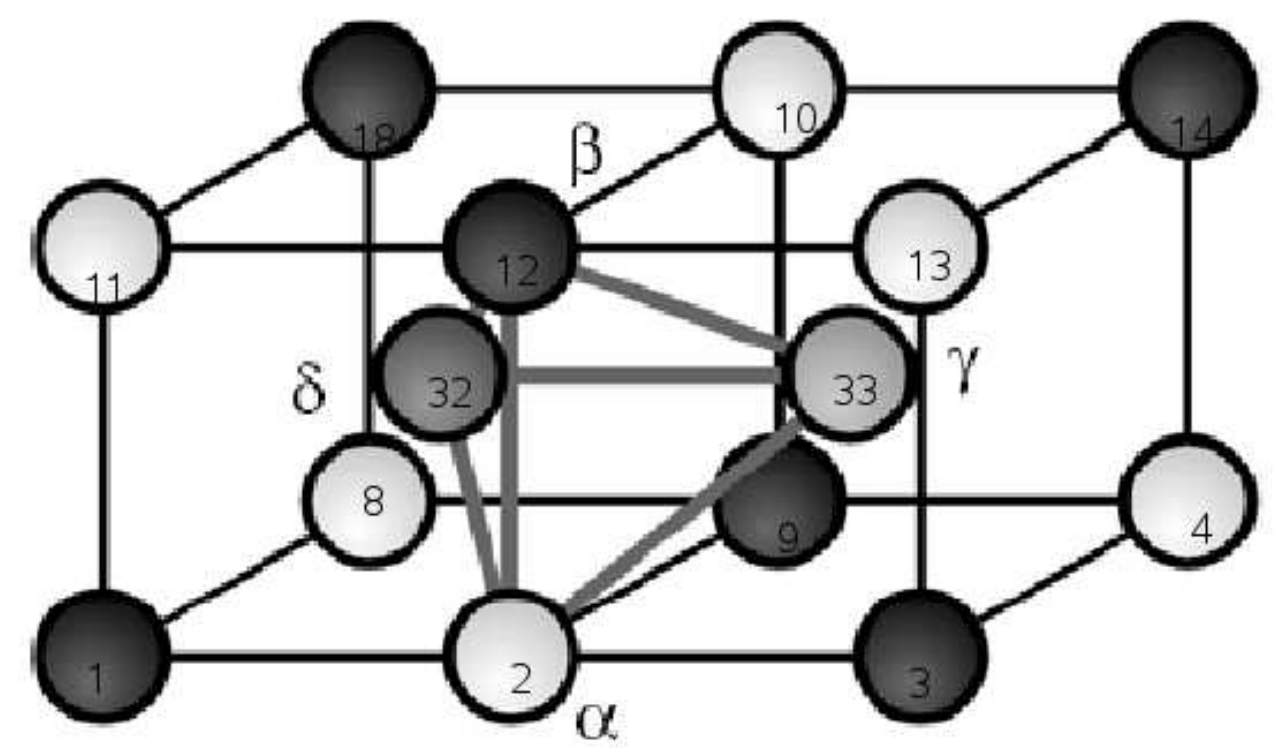

Figura 3.1: A figura mostra duas células BCC. Os sítios numerados com: 1, $2,8,9,10,11,12,18$ são os vértices e 32 o centro da primeira célula; os sítios $2,3,4,9,10,12,13,14$ são vértices e o 33 o centro da segunda célula. As posições $\alpha, \beta, \gamma, \delta$ formam o tetraedro irregular, no sistema BCC. A face inferior do tetraedro é formada por $\alpha, \gamma$ e $\delta$ indicado pelo plano hachurado. A figura corresponde ao composto B2 do sistema Fe-Al. 
Na figura 3.1 temos duas células BCC:representando cada uma delas, os sítios numerados por $1,2,8,9,10,11,12$ e 18 são os vértices e 32 o centro da primeira célula; 2, 3, 4, 9, 10, 12, 13 e 14 são os vértices e 33 o centro da segunda célula. Nesta mesma figura mostramos o cluster básico usado no presente trabalho, onde as posições $\alpha, \beta, \gamma$ e $\delta$ formam o tetraedro irregular, também salientamos uma das faces do tetraedro formado por $\alpha, \gamma$ e $\delta$. A figura mostrada corresponde ao composto B2 do sistema binário Fe-Al. Consideremos agora os sub-cluster formado pelos pares $\alpha-\beta$ e $\gamma-\delta$. Estes átomos estão separados pela distância correspondente a segundos vizinhos na rede BCC. Da mesma forma os pares $\alpha-\gamma, \alpha-\delta, \beta-\gamma$ e $\beta-\delta$ correspondem a pares de primeiros vizinhos[87]. Os sub-cluster do cluster básico, tetraedro irregular são:

1. Pontos.

2. Pares de primeiros vizinhos, que serão representados por: 1viz.

3. Pares de segundos vizinhos, que serão representados por: 2viz.

4. Pontos que formam um triângulo isósceles, que serão representados por: Ti-Is.

5. O tetraedro irregular, que será representado por: Te-Ir.

As configurações de um sub-cluster são definidas de maneira análoga as do cluster. Uma configuração espacial do tetraedro é representado pelo conjunto $\{i, j, k, l\}$ onde $i, j, k$ e $l$ indicam quais espécies ocupam as posições $\alpha, \beta, \gamma$ e $\delta$, respectivamente. Para calcular a entropia configuracional, precisamos estabelecer como está formado o "ensemble microcanônico" e definir as probabilidades de cluster. Isto será feito a seguir.

\subsubsection{Ensemble microcanônico.}

O ensemble microcanônico, $\{\Lambda\}$, do sistema é determinado por todos os arranjos possíveis de clusters no sistema. Seja $q$ do conjunto $\{\lambda\}$, escrito como $q^{\{\lambda\}}$, que representa o número de coordenação do cluster $\{\lambda\}$, isto é, o número de clusters $\{\lambda\}$ por posição de rede em uma determinada estrutura cristalina. O número, $N^{\{\lambda\}}$ de cluster $\{\lambda\}$ na rede é dado portanto por:

$$
N^{\{\lambda\}}=q^{\{\lambda\}} N
$$

onde $N$ foi definido anteriormente como o número de sítios do sistema cristalino. Os valores de $q^{\{\lambda\}}$ são fornecidos na tabela (3.1) para os subclusters do tetraedro irregular. Por exemplo, $q^{\{\lambda\}}$ para o cluster de pares de 
primeira vizinhança é obtido da seguinte forma: o ponto de referência é o sitio $\alpha$, com o plano perpendicular na direção $(0,0,1)$ que o contém. Na figura 3.1 , pode-se contar 4 pares de primeiro vizinhos acima deste plano, e mais 4 pares abaixo do plano dando um total de 8. Este número é dividido por 2, porque a contagem se repete quando tomarmos como referência o segundo ponto pertencente ao par. Finamente dividimos pela quantidade de pares distinguíveis da primeira vizinhança (que são 4) para obter como resultado o valor 1, assim como indicado na tabela (3.1)

\begin{tabular}{|c|c|c|c|}
\hline Cluster $(\lambda)$ & Configurações & $q^{\{\lambda\}}$ & $a^{\{\lambda\}}$ \\
\hline \hline Te-Ir & $\{\alpha, \beta, \gamma, \delta\}$ & 6 & 1 \\
\hline Ti-Is & $\{\alpha, \beta, \gamma\},\{\alpha, \gamma, \delta\},\{\alpha, \beta, \gamma\} \mathrm{e}\{\beta, \gamma, \delta\}$ & 6 & -1 \\
\hline 2viz & $\{\alpha, \beta\} \mathrm{e}\{\gamma, \delta\}$ & $3 / 2$ & 1 \\
\hline 1viz & $\{\alpha, \gamma\},\{\alpha, \delta\},\{\beta, \gamma\} \mathrm{e}\{\beta, \delta\}$ & 1 & 1 \\
\hline Ponto & $\{\alpha\},\{\beta\},\{\gamma\} \mathrm{e}\{\delta\}$ & $1 / 4$ & -1 \\
\hline
\end{tabular}

Tabela 3.1: Número de coordenação $q^{\{\lambda\}}$, coeficientes de Kikuchi-Barker $a^{\{\lambda\}}$ para os sub-clusters do tetraedro irregular BCC. TE-Ir = tetraedro irregular, Ti-Is = triângulo isósceles, 1viz = par de primeiro vizinhos, 2viz = par de segundos vizinhos.

Os coeficientes de Kikuchi-Barker, $a^{\{\lambda\}}$, da tabela (3.1), que serão utilizados mais adiante, são definidos pela relação:

$$
a^{(r)}=1-\sum_{s=0}^{r-1} a^{(4-s)} \frac{q^{(4-s)}}{q^{(r)}} n_{4-s}^{r}
$$

sendo que o $n_{4-s}^{r}$ representa o número de sub-clusters de tipo $\mathrm{r}$ contidos no sub-clusters (4-s), por exemplo, para o caso de $n_{4}^{3}$ representa o número de triângulos contidos no tetraedro.

\subsubsection{Configuração do tetraedro}

Seja $\lambda=\{\alpha, \beta, \gamma, \delta\}$ o cluster básico, na aproximação do tetraedro irregular. A probabilidade de obter uma configuração $\{i, j, k, l\}$ sobre as posições $\alpha, \beta, \gamma$ e $\delta$ é dada por

$$
\rho_{i, j, k, l}^{\alpha, \beta, \gamma, \delta}=\frac{N_{i, j, k, l}^{\alpha, \beta, \gamma} \delta}{q^{\{\lambda\}} N}
$$


onde $N_{i, j, k, l}^{\alpha, \beta, \gamma, \delta}$ é o número de clusters $\lambda$ com a dada configuração $\{i, j, k, l\}$ no sistema. Na estrutura B2, figura 3.1, pela simetria pode-se trocar as posições $i$ e $j$, assim como $k$ e $l$, sem mudar o valor da probabilidade. Podemos obter as probabilidades de sub-cluster a partir das probabilidades de tetraedro com as chamadas "relações de redução" que correspondem a somas parciais sobre os índices das configurações de tetraedro. Para as probabilidades de triângulo temos, por exemplo:

$$
\rho_{i, k, l}^{\alpha, \gamma, \delta}=\sum_{j \prime} \rho_{i, j l, k, l}^{\alpha, \beta, \gamma},
$$

ou ainda:

$$
\rho_{k, i, j}^{\gamma, \alpha, \delta}=\sum_{l l} \rho_{i, j, k, l \prime}^{\alpha, \beta, \gamma, \delta}
$$

As probabilidades dos sub-clusters, portanto, não são independentes e estão relacionadas as probabilidades de tetraedro, "relações de redução". Usando o tetraedro irregular para o sistema BCC binário, podemos definir algumas estruturas com base nas probabilidades de ponto, assim como esta mostrada na tabela (3.2) onde usamos a notação Strukturbericht.

\begin{tabular}{|c|c|}
\hline Nome da estrutura & Restrições sobre as probabilidades de ponto \\
\hline \hline A2 & $\rho_{i}^{(\alpha)}=\rho_{i}^{(\beta)}=\rho_{i}^{(\gamma)}=\rho_{i}^{(\delta)}$ \\
\hline B2 & $\left\{\rho_{i}^{(\alpha)}=\rho_{i}^{(\beta)}\right\} \neq\left\{\rho_{i}^{(\gamma)}=\rho_{i}^{(\delta)}\right\}$ \\
\hline B32 & $\left\{\rho_{i}^{(\alpha)}=\rho_{i}^{(\gamma)}\right\} \neq\left\{\rho_{i}^{(\beta)}=\rho_{i}^{(\delta)}\right\}$ \\
\hline D03 & $\left\{\rho_{i}^{(\alpha)}=\rho_{i}^{(\beta)}\right\} \neq\left\{\rho_{i}^{(\gamma)} \neq \rho_{i}^{(\delta)}\right\}$ \\
\hline
\end{tabular}

Tabela 3.2: Estruturas baseadas nas probabilidades de pontos.

1. A2 - A estrutura desordenada BCC é chamada de A2 quando as posições $\alpha, \beta, \gamma$ e $\delta$ têm a mesma probabilidade de serem ocupadas.

\section{A estrutura (A2)}

Para a rede cúbica de corpo centrado (A2) os dados cristalográficos usados para os cálculos são os seguintes:

Protótipo $=\mathrm{W}$

Símbolo Pearson $=\mathrm{cl} 2$; 
Designação Strukturbericht $=$ A2;

Grupo espacial $=\operatorname{Im} \overline{3} m$;

Numero = 229;

Vetores primitivos:

$A_{1}=\frac{1}{2}(-a \mathbf{X}+a \mathbf{Y}+a \mathbf{Z})$

$A_{2}=\frac{1}{2}(+a \mathbf{X}-a \mathbf{Y}+a \mathbf{Z})$

$A_{3}=\frac{1}{2}(+a \mathbf{X}+a \mathbf{Y}-a \mathbf{Z})$

Vetor Base: $\mathbf{B}_{1}=0(\mathrm{~W})$.

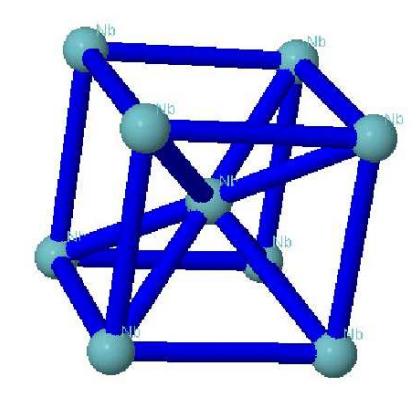

Figura 3.2: Rede cúbica de corpo centrado (A2).

2. B2 - A estrutura ordenada BCC é chamada de B2 quando as posições $\alpha$ e $\beta$ têm a mesma probabilidade de serem ocupadas, assim como as posições $\gamma$ e $\delta$, estas últimas, sendo, porém diferentes das anteriores.

\section{A estrutura FeAl (B2)}

Para a estrutura FeAl (B2) foram utilizados os seguintes dados cristalográficos: Protótipo $=\mathrm{CsCl}$;

Símbolo Pearson $=\mathrm{cP} 2$;

Designação Strukturbericht $=$ B2;

Grupo espacial $=\operatorname{Pm} \overline{3} m$;

Numero $=221$;

Vetores primitivos:

$A_{1}=(+a \mathbf{X})$ 


$$
\begin{aligned}
& A_{2}=(+a \mathbf{Y}) \\
& A_{3}=(+a \mathbf{Z}) ; \\
& \text { Vetor Base: } \mathbf{B}_{1}=0(\mathrm{Cs})(1 \mathrm{a}) \\
& \mathbf{B}_{2}=\frac{1}{2}\left(\mathbf{A}_{\mathbf{1}}+\mathbf{A}_{\mathbf{2}}+\mathbf{A}_{\mathbf{3}}\right)=\frac{a}{2}(\mathbf{X}+\mathbf{Y}+\mathbf{Z})(\mathrm{Cl})(1 \mathrm{~b}) .
\end{aligned}
$$

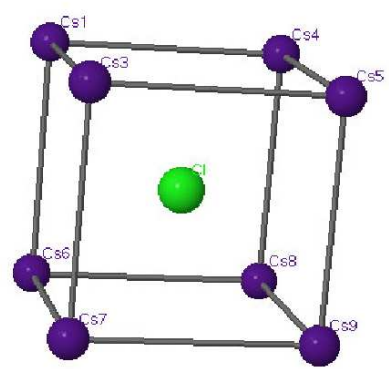

Figura 3.3: Rede cúbica de Fase centrada (B2).

3. B32 - A estrutura ordenada BCC é chamada de B32 quando as posições $\alpha$ e $\gamma$ têm a mesma probabilidade de serem ocupadas, assim como as posições $\beta$ e $\gamma$, sendo porém diferentes das anteriores.

\section{A estrutura FeAl (B32)}

Para a estrutura FeAl (B32) foram utilizados os seguintes dados cristalográficos, dados obtidos de [91]: Protótipo = NaTi;

Símbolo Pearson $=\mathrm{cF} 16$;

Designação Strukturbericht $=$ B32;

Grupo espacial $=F d \overline{3} m$;

Numero $=227$

Vetores primitivos:

$A_{1}=\frac{1}{2}(+a \mathbf{Y}+a \mathbf{Z}) ;$
$A_{2}=\frac{1}{2}(+a \mathbf{X}+a \mathbf{Z}) ;$
$A_{3}=\frac{1}{2}(+a \mathbf{X}+a \mathbf{Y}) ;$

Vetor Base: $\mathbf{B}_{1}=\frac{1}{8}\left(\mathbf{A}_{\mathbf{1}}+\mathbf{A}_{\mathbf{2}}+\mathbf{A}_{\mathbf{3}}\right)=\frac{a}{8}(\mathbf{X}+\mathbf{Y}+\mathbf{Z})(\mathrm{Na})(8 \mathrm{a})$

$\mathbf{B}_{2}=\frac{-1}{8}\left(\mathbf{A}_{\mathbf{1}}+\mathbf{A}_{\mathbf{2}}+\mathbf{A}_{\mathbf{3}}\right)=\frac{-a}{8}(\mathbf{X}+\mathbf{Y}+\mathbf{Z})(\mathrm{Na})(8 \mathrm{a})$ 


$$
\begin{aligned}
& \mathbf{B}_{3}=\frac{3}{8}\left(\mathbf{A}_{\mathbf{1}}+\mathbf{A}_{\mathbf{2}}+\mathbf{A}_{\mathbf{3}}\right)=\frac{3 a}{8}(\mathbf{X}+\mathbf{Y}+\mathbf{Z})(\mathrm{Ti})(8 \mathrm{~b}) \\
& \mathbf{B}_{4}=\frac{-3}{8}\left(\mathbf{A}_{\mathbf{1}}+\mathbf{A}_{\mathbf{2}}+\mathbf{A}_{\mathbf{3}}\right)=\frac{-3 a}{8}(\mathbf{X}+\mathbf{Y}+\mathbf{Z})(\mathrm{Ti})(8 \mathrm{~b})
\end{aligned}
$$

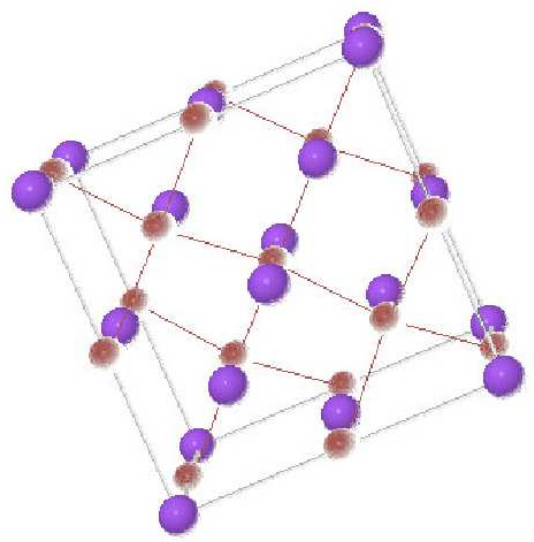

Figura 3.4: Liga binária NaTi com a fase (B32).

4. D03 - A estrutura ordenada BCC é chamada de D03 quando as posições $\alpha, \beta$ têm a mesma probabilidade de serem ocupadas mas que diferem da probabilidade da posição $\gamma$ e da probabilidade da posição $\delta(\gamma \neq \delta)$.

\section{A estrutura $\mathrm{Fe}_{3} \mathrm{Al}-\left(\mathrm{D0_{3 }}\right)$}

Representação da estrutura cristalina da liga binária $\mathrm{Fe}_{3} \mathrm{Al}$ com a fase (D03). Representação efetuada através do software Jmol.Os dados a seguir foram obtidos de [91].

Protótipo $=\mathrm{Fe}_{3} \mathrm{Al}$;

Símbolo Pearson $=\mathrm{cF} 16$;

Designação Strukturbericht $=\mathrm{D0}_{3}$;

Grupo espacial $=F m \overline{3} m$;

Numero $=225$;

Outros elementos com esta estrutura $=\mathrm{BiFe}_{3}$; Vetores primitivos:

$A_{1}=\frac{1}{2}(+a \mathbf{Y}+a \mathbf{Z})$

$A_{2}=\frac{1}{2}(+a \mathbf{X}+a \mathbf{Z})$

$A_{3}=\frac{1}{2}(+a \mathbf{X}+a \mathbf{Y})$

Vetor Base: $\mathbf{B}_{1}=0(\mathrm{Al})(4 \mathrm{a})$

$\mathbf{B}_{2}=\frac{1}{2}\left(-\mathbf{A}_{\mathbf{1}}+\mathbf{A}_{\mathbf{2}}+\mathbf{A}_{\mathbf{3}}\right)=\frac{a}{2}(\mathbf{X})(\mathrm{Fe})(4 \mathrm{~b})$ 


$$
\begin{aligned}
& \mathbf{B}_{3}=\frac{-1}{4}\left(\mathbf{A}_{\mathbf{1}}+\mathbf{A}_{\mathbf{2}}+\mathbf{A}_{\mathbf{3}}\right)=\frac{-a}{4}(\mathbf{X}+\mathbf{Y}+\mathbf{Z})(\mathrm{Fe})(8 \mathrm{c}) \\
& \mathbf{B}_{4}=\frac{1}{4}\left(\mathbf{A}_{\mathbf{1}}+\mathbf{A}_{\mathbf{2}}+\mathbf{A}_{\mathbf{3}}\right)=\frac{a}{4}(\mathbf{X}+\mathbf{Y}+\mathbf{Z})(\mathrm{Fe})(8 \mathrm{c})
\end{aligned}
$$

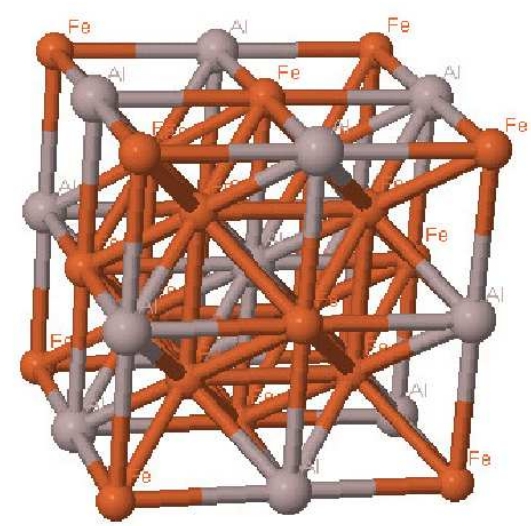

Figura 3.5: Liga binária $\mathrm{Fe}_{3} \mathrm{Al}$ com a fase (D03).

Em um sistema binário A-B é possível definir seis configurações não equivalentes:

1. A2 da estequiometria A.

2. A2 da estequiometria B.

3. D03 da estequiometria $A_{3} B$.

4. D03 da estequiometria $A B_{3}$.

5. B2 da estequiometria AB.

6. B32 da estequiometria AB.

\subsubsection{Entropia}

O princípio fundamental do CVM é calcular a entropia configuracional do "ensemble micro-canônico" do sistema em termos das probabilidades de cluster. A entropia do sistema é dada por

$$
S=-q^{\{\lambda\}} N K_{B} \sum_{i, j, k, l} \rho_{i, j, k, l}^{\alpha, \beta, \gamma, \delta} \ln \rho_{i, j, k, l}^{\alpha, \beta, \gamma, \delta}-N K_{B} \sum_{v \subset \lambda} q^{v} a^{v} \sum_{c o n f i} \rho^{v} \ln \rho^{v},
$$


onde $K_{B}=8.3145 \mathrm{~J} / \mathrm{mol}(K)$ é a constante de Boltzmann. A primeira somatória da equação (3.23) considera todas as configurações do tetraedro, $\{\lambda\}$, enquanto a segunda é sobre todos os sub-clusters de $\{\lambda\}$ e suas configurações (confi). Os $a^{v}$ são os "coeficientes de Kikuchi-Barker"para os sub-clusters de $\{\lambda\}$, indicados na tabela 3.1. Observe que a soma é realizada sobre todos os sub-clusters $v$ contidos em $\lambda$.

\subsubsection{Energia Interna}

Uma vez definido o cluster básico e suas probabilidades, podemos escrever a energia livre do sistema em função dessas probabilidades. A energia livre é dada por

$$
U^{\Lambda}=q^{\{\lambda\}} N \sum_{i, j, k, l} \varepsilon_{i, j, k, l}^{\{\lambda\}} \rho_{i, j, k, l}^{\{\lambda\}}
$$

onde os termos $\varepsilon_{i, j, k, l}^{\{\lambda\}}$ são auto-energias da configuração $\{i, j, k, l\}$ no tetraedro $\{\lambda\}$, também chamadas de interação de cluster. Para o caso do tetraedro irregular, as interações podem ser escritas em forma de combinações lineares de interações de pares e interações de maior ordem,

$$
\begin{aligned}
\varepsilon_{i, j, k, l}^{\{\lambda\}} & =\frac{1}{6}\left(\varepsilon_{i, k}^{(1)}+\varepsilon_{i, l}^{(1)}+\varepsilon_{j, k}^{(1)}+\varepsilon_{j, l}^{(1)}\right)+\frac{1}{4}\left(\varepsilon_{i, j}^{(2)}+\varepsilon_{k, l}^{(2)}\right) \\
& +\frac{1}{2}\left(\widetilde{\varepsilon}_{i, k, j}+\widetilde{\varepsilon}_{i, l, j}+\widetilde{\varepsilon}_{k, i, l}+\widetilde{\varepsilon}_{k, j, l}\right)+\widetilde{\varepsilon}_{i, j, k, l}
\end{aligned}
$$

$\mathrm{Na}$ expressão 3.25, as interações entre pares são indicadas pelos sobrescritos (1) para os primeiros vizinhos, (2) para os segundos vizinhos. Os outros parâmetros, indicados por um til $(\sim)$, são as interações entre triângulos e entre conjuntos de quatros átomos, ressaltando que não devem ser levadas em conta, novamente, as interações de pares. As frações são necessárias porque os pares de primeiro vizinhos são compartilhados entre 6 tetraedros, os pares de segundos vizinhos são compartilhados entre 4 tetraedros e finalmente os triângulos são compartilhados entre 2 tetraedros. O sistema de equações (uma equação para cada configuração $\{i, j, k, l\}$ ) da expressão 3.25 pode ser simplificado pela definição da mistura mecânica dos componentes como estado de referência para a energia. Isto equivale a substituir os parâmetros 
em 3.25 por,

$$
\begin{aligned}
\widetilde{w}_{i, j, k, l} & =\widetilde{\varepsilon}_{i, j, k, l}-\frac{1}{4} \sum_{m=i, j, k, l} \widetilde{\varepsilon}_{m, m, m, m} \\
\widetilde{w}_{i, j, k} & =\widetilde{\varepsilon}_{i, j, k}-\frac{1}{3} \sum_{n=i, j, k} \widetilde{\varepsilon}_{n, n, n} \\
\widetilde{w}_{i, j, k} & =\widetilde{\varepsilon}_{i, j}^{(d)}-\frac{1}{2} \widetilde{\varepsilon}_{i, i}^{(d)}-\frac{1}{2} \widetilde{\varepsilon}_{j, j}^{(d)}
\end{aligned}
$$

Uma simplificação maior pode ser obtida se considerarmos as degenerescências das interações de cluster devido á simetria da rede BCC. Após a consideração de todos os elementos de simetria da rede BCC conclui-se que um sistema binário apresenta 4 parâmetros de interação não equivalentes. Por razões históricas prefere-se trabalhar com as contribuições dos pares $(\mathrm{d}=1,2)$, $w_{A B}^{1}$ e $w_{A B}^{2}$, que correspondem ás contribuições da primeira e segunda vizinhança, respectivamente, completando-se o sistema de equações com duas interações relativas a configuração de tetraedro, $w_{A B A B}$ e $w_{A B B B}$, que correspondem as contribuições das estruturas B32 (AB) e D03 $\left(\mathrm{AB}_{3}\right)$, respectivamente. Note que as energias de formação das duas fases A2 são nulas devido á escolha do estado de referência. Estes serão chamados de parâmetros de interação binárias do sistema. As energias de formação dos compostos calculados com o método FP-LAPW podem ser relacionadas aos parâmetros de interação pelo seguinte sistema de equações:

$$
\left(\begin{array}{c}
U_{D 03-A_{3} B}^{f} \\
U_{B 2}^{f} \\
U_{B 32}^{f} \\
U_{D 03-A B_{3}}^{f}
\end{array}\right)=6 N\left(\begin{array}{cccc}
0 & 0 & 1 / 4 & 1 / 3 \\
0 & 0 & 0 & 2 / 3 \\
1 & 0 & 1 / 2 & 1 / 3 \\
0 & 1 & 1 / 4 & 1 / 3
\end{array}\right) \times\left(\begin{array}{c}
\widetilde{w}_{A B A B} \\
\widetilde{w}_{A B B B} \\
\widetilde{w}_{A B}^{(2)} \\
\widetilde{w}_{A B}^{(1)}
\end{array}\right)
$$

Desenvolvendo a equação (3.27), obteremos 4 equações correspondentes aos parâmetros de interações binárias, que são:

$$
\begin{gathered}
4 \widetilde{w}_{A B}^{1}=U_{B 2}^{f}, \\
2 \widetilde{w}_{A B}^{1}+\frac{3}{2} \widetilde{w}_{A B}^{2}=U_{D 03-A_{3} B}^{f}, \\
2 \widetilde{w}_{A B}^{1}+3 \widetilde{w}_{A B}^{2}+6 \widetilde{w}_{A B A B}=U_{B 32}^{f}, \\
2 \widetilde{w}_{A B}^{1}+\frac{3}{2} \widetilde{w}_{A B}^{2}+6 \widetilde{w}_{A B B B}=U_{D 03-A B_{3}}^{f} .
\end{gathered}
$$


Para um sistema ternário A-B-C, com estrutura BCC, são necessários 18 parâmetros de interações. Destes 12 correspondem aos parâmetros de interações binárias, que são 4 parâmetros do binário A-B, 4 do binário B-C mais 4 do binário A-C. As configurações ternárias do sistema fornecem mais seis parâmetros, os quais são obtidos desenvolvendo-se a equação (3.27) para um sistema ternário, e que são:

$$
\begin{gathered}
6 \widetilde{w}_{A A B C}+\frac{3}{2} \widetilde{w}_{B C}^{2}+2\left\{\widetilde{w}_{A B}^{1}+\widetilde{w}_{C A}^{1}\right\}=U_{L 2_{1}-A 2 C B}^{f}, \\
6 \widetilde{w}_{A B A C}+\frac{3}{2}\left\{\widetilde{w}_{A B}^{2}+\widetilde{w}_{C A}^{2}\right\}+\left\{\widetilde{w}_{A B}^{1}+\widetilde{w}_{C A}^{1}+\widetilde{w}_{b C}^{1}\right\}=U_{F 4 \overline{3} m-A 2 C B}^{f}, \\
6 \widetilde{w}_{B B A C}+\frac{3}{2} \widetilde{w}_{C A}^{2}+2\left\{\widetilde{w}_{B C}^{1}+\widetilde{w}_{A B}^{1}\right\}=U_{L 2_{1}-B 2 C A}^{f}, \\
6 \widetilde{w}_{B C B A}+\frac{3}{2}\left\{\widetilde{w}_{A C}^{2}+\widetilde{w}_{A B}^{2}\right\}+\widetilde{w}_{A B}^{1}+\widetilde{w}_{C A}^{1}+\widetilde{w}_{B C}^{1}=U_{F 4 \overline{3} m-B 2 C A}^{f}, \\
6 \widetilde{w}_{C C A B}+\frac{3}{2} \widetilde{w}_{A B}^{2}+2\left\{\widetilde{w}_{C B}^{1}+\widetilde{w}_{C A}^{1}\right\}=U_{L 2_{1}-C 2 A B}^{f}, \\
6 \widetilde{w}_{C A C B}+\frac{3}{2}\left\{\widetilde{w}_{B C}^{2}+\widetilde{w}_{C A}^{2}\right\}+\widetilde{w}_{A B}^{1}+\widetilde{w}_{C A}^{1}+\widetilde{w}_{B C}^{1}=U_{F 4 \overline{3} m-C 2 A B}^{f} .
\end{gathered}
$$

Note que nas equações (3.34) e (3.33) a composição é a mesma, isto é $A_{2} C B$, mas as estruturas são diferentes sendo a primeira chamada de $L 2_{1}$ e a segunda de $F 4_{3} m$, respectivamente. Analogamente isto acontece com as composições $\mathrm{B}_{2} \mathrm{CA}$ e $\mathrm{C}_{2} \mathrm{AB}$. Para nosso sistema $\mathrm{Fe}-\mathrm{Al}-\mathrm{Cr}$, substituiremos $A=F e, B=A l$ e $C=C r$ nas equações 3.32 à 3.37 .

\section{A estrutura Heusler- $\left(L 2_{1}\right)$}

Os dados a seguir foram obtidos de [91]. Protótipo $=\mathrm{AlCu}_{2} \mathrm{Mn}$;

Símbolo Pearson = cF16;

Designação Strukturbericht $=L 2_{1}$;

Grupo espacial $=F m \overline{3} m$;

Numero $=216$;

Vetores primitivos:

$A_{1}=\frac{1}{2}(+a \mathbf{Y}+a \mathbf{Z})$

$A_{2}=\frac{1}{2}(+a \mathbf{X}+a \mathbf{Z})$; 
$A_{3}=\frac{1}{2}(+a \mathbf{X}+a \mathbf{Y})$

Vetor Base: $\mathbf{B}_{1}=0(\mathrm{Mg})(4 \mathrm{a})$

$\mathbf{B}_{2}=\frac{1}{2}\left(-\mathbf{A}_{\mathbf{1}}+\mathbf{A}_{\mathbf{2}}+\mathbf{A}_{\mathbf{3}}\right)=\frac{a}{2}(\mathbf{X})(\mathrm{Ag})(4 \mathrm{~b})$

$\mathbf{B}_{3}=\frac{1}{4}\left(\mathbf{A}_{\mathbf{1}}+\mathbf{A}_{\mathbf{2}}+\mathbf{A}_{\mathbf{3}}\right)=\frac{a}{4}(\mathbf{X}+\mathbf{Y}+\mathbf{Z})(\mathrm{As})(4 \mathrm{c})$

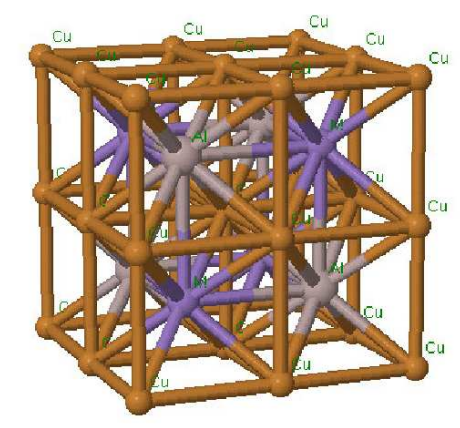

(a)

Figura 3.6: Ligas ternárias com a designação strukturberich $L 2_{1}$.

\subsubsection{Energia Livre}

Com a definição da energia interna, podemos construir a energia livre, F, do sistema como:

$$
F=U-T S+\sum_{i, j, k, l=F e, M o} \frac{\mu_{i}^{*}+\mu_{j}^{*}+\mu_{k}^{*}+\mu_{l}^{*}}{4} \rho_{i, j, k, l}^{\alpha \beta \gamma \delta}
$$

Nesta expressão, S representa a entropia configuracional de uma rede cúbica de corpo centrado na aproximação de cluster do tetraedro irregular, o qual é encontrado em geral na referência [92]. Variáveis $\mu_{m}^{*}$ representa o potêncial quimico da componente $m$ no estado de referência baricentrico, definido pela transformação:

$$
\mu_{M o}^{*}=\frac{\mu_{A}-\mu_{B}}{2}=-\mu_{B}^{*}
$$

onde $\mu_{A}$ e $\mu_{B}$ são os potênciais quimicos de A e B em algum estado de referência (em geral o Elemento padrão de referência, SER[10]). 
Usando a Identidade:

$$
U-T S=N \sum_{i=M o, F e} \mu_{i} x_{i}
$$

e substituindo 3.39, em 3.38, nos obtemos:

$$
F=N\left(\frac{\mu_{A}+\mu_{B}}{2}\right)
$$

\subsubsection{Minimização da energia livre a temperaturas fini- tas}

Nesta seção, procuramos descrever um dos métodos de minimização da energia livre para obtenção do estado de equilíbrio termodinâmico. O método foi desenvolvido por Kikuchi em 1974 [62], para o caso puramente configuracional, e foi batizado de NIM (Natural Interation Method). Outro método de minimização comumente utilizado é o método de Newton-Raphson (NR) [63]. De maneira geral, podemos destacar as seguintes vantagens e desvantagens dos dois métodos:

- O algaritmo NR converge rapidamente para uma solução, desde que a estimativa inicial não esteja muito longe da solução. Caso contrário, o sistema de equações irá possivelmente divergir, resultando em erros de ponto flutuante.O raio de convergência para a solução diminui com o aumento do número de componentes (ou seja, da dimensão do sistema de equações).

- O algoritmo NIM converge lentamente para uma solução. No entanto, a convergência é garantida, independentemente da estimativa inicial e do número de componentes.

O algoritmo escolhido para o presente trabalho foi o NIM. O NIM é um algoritmo iterativo autoconsistente capaz de fornecer as probabilidades e as funções termodinâmicas no equilíbrio.

Um exemplo do processo de minimizar a energia livre de Gibbs sob a restrição dos potenciais químicos constantes para o caso de um sistema binário é ilustrado em [1], descreveremos brevemente o processo a seguir.

A equação 3.38 é minimizada utilizando o algoritmo dedicado chamado "Natural Iteration Method", NIM [62]. Para valores dados de $T$ e $\left\{\mu_{A}^{*}\right\}$ podemos eventualmente obter diferentes soluções para o minimo (global ou 
local) de $F^{3}$ usando uma escolha conveniente de condições iniciais. Supondo a convergência do algoritmo para diferentes phases (por exemplo, $\lambda$ e $\phi$ ), a condição de equilibrio será dado por:

$$
F^{\lambda}=F^{\phi}
$$

Um calculo do diagrama de fases corresponde ao mapeamento de todos as possíveis soluções da equação 3.42 como função de $T$ e $\mu_{A}^{*}$, para todos pares de configurações estaveis, $\lambda$ e $\phi$.

Nos sistemas contendo gaps de imiscibilidade há diferentes soluções correspondendo a fases com idênticas simetris e diferentes composições. Próximo ao máximo do gap a diferença na composição desaparece, então a obtenção da solução da equação 3.42 é espinhosa nesta região. Todos os cálculos aqui reportados usando a temperatura esta a $1 \mathrm{~K}$ do máximo do gap, sempre começando do primeiro ponto convergido, correspondendo a temperatura mais alta, continuando para baixo na escala de temperatura. Este primeiro ponto convergido é determinado por tentativa e erro e é incluido no plot.

\subsubsection{Componentes com momento magnético}

Para o caso com componentes magnéticos, primeiro redefinimos a Hamiltoniana dentro da estrutura BCC que estamos utilizando, isto e, para o tetraedro irregular a Hamiltoniana de Ising vai ser dada por

$$
\begin{aligned}
E= & q^{B C C}\left\{\sum_{\text {cluster }} \frac{J^{1}\left(\sigma_{\alpha} \sigma_{\gamma}+\sigma_{\alpha} \sigma_{\delta}+\sigma_{\beta} \sigma_{\gamma}+\sigma_{\beta} \sigma_{\delta}\right)}{6 s^{2}}+\right. \\
& \left.\frac{J^{2}\left(\sigma_{\alpha} \sigma_{\beta}+\sigma_{\gamma} \sigma_{\delta}\right)}{4 s^{2}}\right\}-\frac{g \mu_{B} H_{e}}{s} \sum_{\text {cluster }} \frac{\left(\sigma_{\alpha}+\sigma_{\beta}+\sigma_{\gamma}+\sigma_{\delta}\right)}{4}
\end{aligned}
$$

Neste caso, $\sigma_{x}(x=\alpha, \beta, \gamma, \delta)$ são os operadores de projeção de spin, $J^{1}$ representa a energia de interação magnética entre primeiros vizinhos, $J^{2}$ a interação magnética entre segundos vizinhos, $q^{B C C}=6$ é o número de coordenação do tetraedro, $H_{e}$ representa o módulo de um campo magnético externo, $g$ é a razão giromagnético, $\mu_{B}$ é o magneton de Bohr e o $s$ é o número quântico de spin; neste trabalho utilizaremos o valor $s=1$ para descrever o magnetismo do $F e$. Fazendo uma média da hamiltoniana sobre todos os tetraedros do sistema, obtemos o funcional $F$

\footnotetext{
${ }^{3}$ Nos presentes cálculos a condição de convergência foi de uma diferença menor que $10^{-4} k_{B} . K$ entre dois valores de $F$ obtidos em subsequentes interações do NIM ( $1 k_{B} . K$ $\left.=8.3145 \mathrm{~J} \mathrm{~mol}^{-1}\right)$.
} 


$$
F\left(T, H\left[\begin{array}{l}
\alpha, \beta, \gamma, \delta \\
i, j, k, l
\end{array}\right]\right)=N\left\{\sum_{i, j, k, l=1}^{2 s+1}\left(q^{\alpha, \beta, \gamma, \delta} \varepsilon_{i, j, k, l}^{\alpha, \beta, \gamma, \delta}-\mu_{i, j, k, l}^{*}\right) \rho_{i, j, k, l}^{\alpha, \beta, \gamma, \delta}\right\}-T S^{\alpha, \beta, \gamma, \delta}
$$

com:

$$
\varepsilon_{i, j, k, l}^{\alpha, \beta, \gamma, \delta}=\frac{J^{(1)}\left(s_{i} s_{k}+s_{i} s_{l}+s_{j} s_{k}+s_{j} s_{l}\right)}{6 s^{2}}+\frac{J^{(2)}\left(s_{i} s_{j}+s_{k} s_{l}\right)}{4 s^{2}}+\varepsilon_{\text {configuracional }}
$$

Note que $\varepsilon_{\text {configuracional }}$ foi definida em (3.27). Lembramos que:

$$
U=6 \times \varepsilon_{i, j, k, l}^{\alpha, \beta, \gamma, \delta}
$$

onde $U$ é a energia de formação calculada a partir da energia total, $E_{T}$, obtida no cálculo de estrutura eletrônica utilizando o método FP-LAPW. 


\section{Capítulo 4}

\section{Resultados e \\ Discussões: 01 - Estruturas de Referência (Sistemas Unários)}

Neste capítulo apresentaremos inicialmente os critérios para obter a convergência na energia total $\left(\mathrm{E}_{T}\right)$ bem como os parâmetros de controle para se obter o estado fundamental de cada sistema estudado com o método FPLAPW. Os resultados apresentados e discutidos para os diagramas de fases são obtidos utilizando o funcional de troca - correlação GGA-PBE, mostraremos o motivo da escolha deste funcional. Algumas propriedades mecânicas como o volume de equilíbrio e o "bulk modulus" são mostrados e comparados com seus respectivos valores experimentais durante o processo de otimização estrutural. Os resultados para os sistemas unários utilizado como referência para a construção dos diagramas de fases será discutido neste capítulo:

\subsection{Convergência na energia total}

No capítulo II indicamos que, para o controle do critério de convergência na $E_{T}$ utilizando o método FP-LAPW na versão WIEN2K pode-se variar três parâmetros: $R K_{\max }, L_{\max }$ e o números de pontos-K na primeira zona de Brillouin. Destes parâmetros escolhemos um para que seja o primeiro a convergir enquanto os outros são mantidos fixos. Analogamente repetimos o procedimento com o segundo parâmetro e depois com o terceiro. Desta forma temos vários caminhos alternativos para chegar á convergência do sistema. $\mathrm{O}$ resultado final, entretanto, deve independer do caminho utilizado.

Neste trabalho, utilizamos $10^{-06}$ Rydberg como critério de convergência final na $E_{T}$ para cada processo autoconsistente. O procedimento utilizado será 
agora brevemente descrito e ilustrado com os casos unários utilizados como referência neste estudados.

Na figura 4.1 utilizamos o caso do Fe na estrutura BCC com o cálculo relativístico com polarização de spin, mostramos o gráfico obtido durante o processo da otimização estrutural. Na figura (4.5-a), aumentamos o valor de $R K_{\max }$, fixando $L_{\max }=10$ e 20 pontos-K, até obter a convergência. Nota-se uma grande variação da $E_{T}$ ao incrementar o $R K_{\max }$ de 7 para 8 e uma variação menor de 9 para 10. Utilizamos esses valores iniciais porque estamos estudando sistemas metálicos e que foram determinados pela nossa experiência anterior. Posteriormente fixamos o $R K_{\max }=10$ e incrementamos o $L_{\max }$ mantendo fixos os 20 pontos-K. O resultado esta mostrado na figura (4.5b). Observa-se que $E_{T}$ varia muito ao incrementar $L_{\max }$ de 8 até 10 e varia muito pouco ao incrementar o $L_{\max }$ a partir de 10 , o que nos permite escolher $L_{\max }=10$ ou 11 para a etapa final do procedimento. Finalmente fixamos o $R K_{\max }=10$ e $L_{\max }=10$, obtidos das etapas anteriores e o desenvolvimento da energia em função dos pontos-K é mostrada na figura (4.5c). Observa-se a convergência final do sistema. Note-se que o sistema está convergido ao trabalhar com 286 pontos-K ou com 364 pontos-K. A resolução da escala de $E_{T}$ vai se tornando sucessivamente mais fina quando se passa da figura (4.5-a) para (4.5-b) e deste para (4.5-c). Este é um indicativo importante da convergência do cálculo. O uso de parâmetros altos para $R K_{\max }, L_{\max }$ e número de pontos-K implica no aumento do custo computacional do cálculo, desta forma a escolha dos valores finais para os mesmos deve levar em conta uma relação do tipo custo-benefício. Neste exemplo apresentado, escolhe-se $R K_{\max }=10, L_{\max }=10$ e 286 pontos-K, já que estes valores são os menores para que o sistema esteja convergido. O procedimento descrito acima é feito apenas uma vez para e estrutura estudada, por exemplo, para o $\mathrm{Fe}$, mantendo-se o valor do parâmetro de rede fixo. Finalizando o procedimento, $R K_{\max }, L_{\max }$ e os números de pontos-K são mantidos constantes e o parâmetro de rede é variado. A curva $E_{T}$ versus volume (4.1) se assemelha a uma parábola e o ponto mínimo define o valor de equilíbrio do parâmetro de rede e a $E_{T}$ de equilíbrio para a presente estrutura. Este procedimento, foi testado de forma iterativa em todos os sistemas Fe-Al-Cr apresentados neste trabalho. Um procedimento análogo é mostrado nas figuras 4.2, 4.3 e 4.4. 
Tabela 4.1: Sistema Unário de referência. Onde: $V_{0}$ é o volume minimo dado em u.a., BP á derivada do bulk modulus e $E_{0}$ é a energia minima do processo de otimização dado em Ry.

\begin{tabular}{cccccc}
\hline Sistema & Estrutura & $V_{0}$ & $\mathrm{~B}(\mathrm{GPa})$ & $\mathrm{BP}$ & $E_{0}$ \\
\hline $\mathrm{Fe}(\mathrm{FM})$ & $\mathrm{A} 2$ & 153.113 & 178.876 & 6.938 & -2545.612423 \\
$\mathrm{Al}(\mathrm{FM})$ & $\mathrm{A} 1$ & 111.219 & 76.862 & 4.757 & -485.644212 \\
$\mathrm{Al}(\mathrm{FM})$ & $\mathrm{A} 2$ & 114.091 & 68.683 & 4.564 & -485.637156 \\
$\mathrm{Cr}(\mathrm{AFM})$ & $\mathrm{A} 2$ & 156.102 & 259.644 & 4.404 & -2101.783673 \\
\hline
\end{tabular}

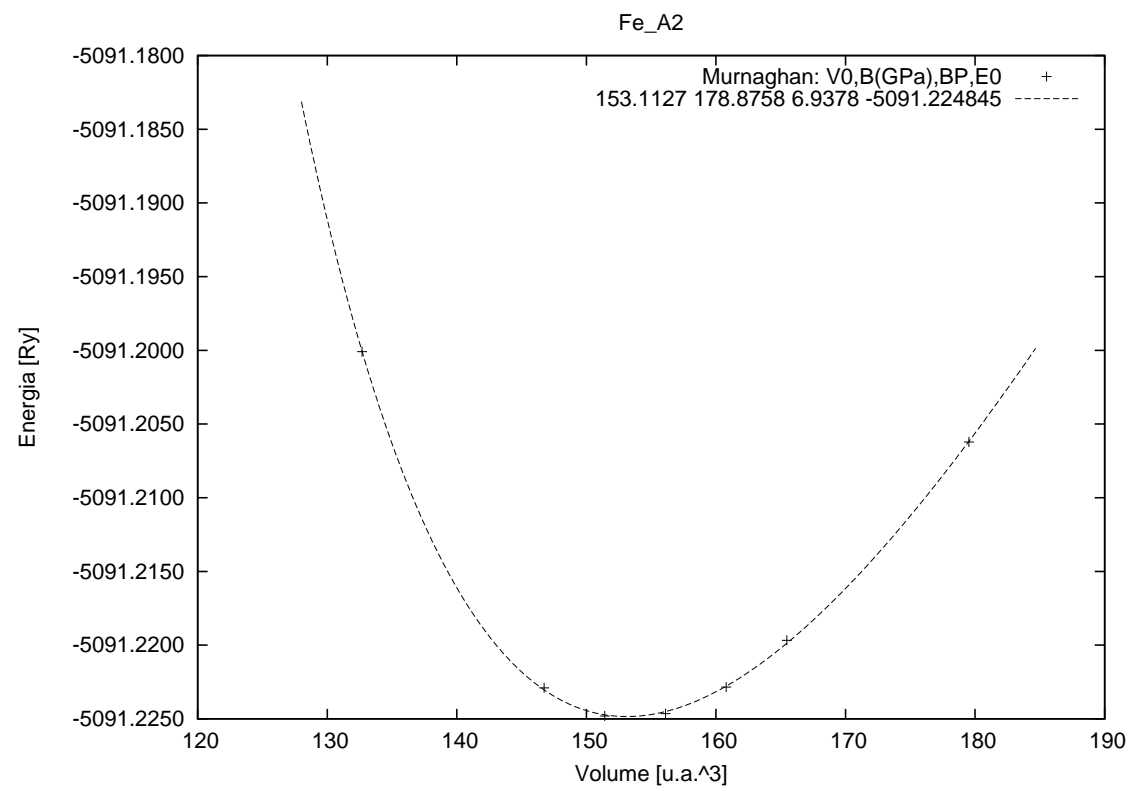

Figura 4.1: Otimização estrutural do Ferro cúbico de corpo centrado (Fe-A2) para o caso FP-LAPW.

Nossa conclusão deste estudo é que basta apenas o procedimento de otimização descrito acima e ilustrados nas figuras (4.5), para encontrar o melhor conjunto de parâmetros, $R K_{\max }, L_{\max }$ e número de pontos-K. O parâmetro de rede otimizado encontrado é mostrado na Tabela 4.2 Energia total calculada para os três sistemas unários 4.2 formando a base de referência para a expansão de cluster aplicada ao sistema Fe-Al-Cr. Para todos os cálculos $\mathrm{RKmax}=\mathrm{Lmax}=10$, número de pontos $\mathrm{K}$ na zona irredutível de Brillouin foi de 286. O momento magnético médio no átomo (MM) é apresentado (em magneton de Bohr, $\mu_{B}$ ). Os parâmetros de rede são expressados em nm e os valores da das energias são representados em Rydberg (Ry) por fórmula unitária $\left(1 \mathrm{Ry}=2.1798741 \times 10^{-18} \mathrm{~J}\right.$. 


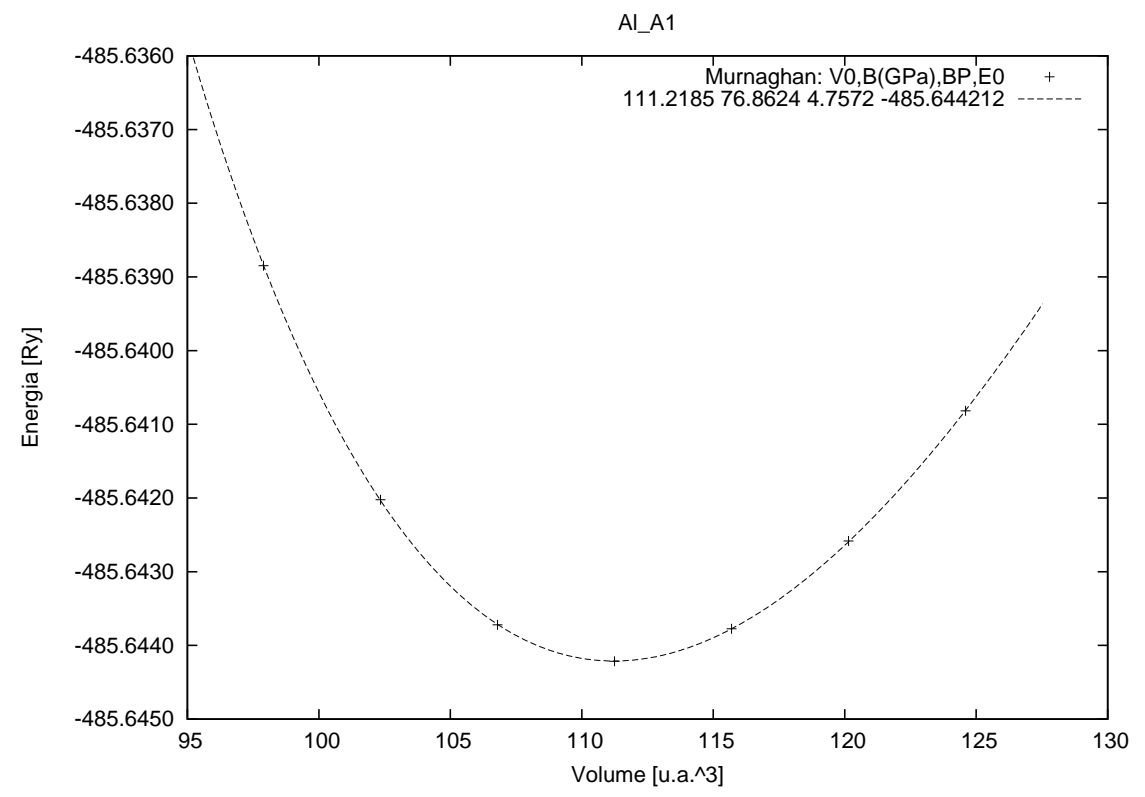

Figura 4.2: Otimização estrutural do Alumínio cúbico de face centrada (AlA1) para o caso FP-LAPW.

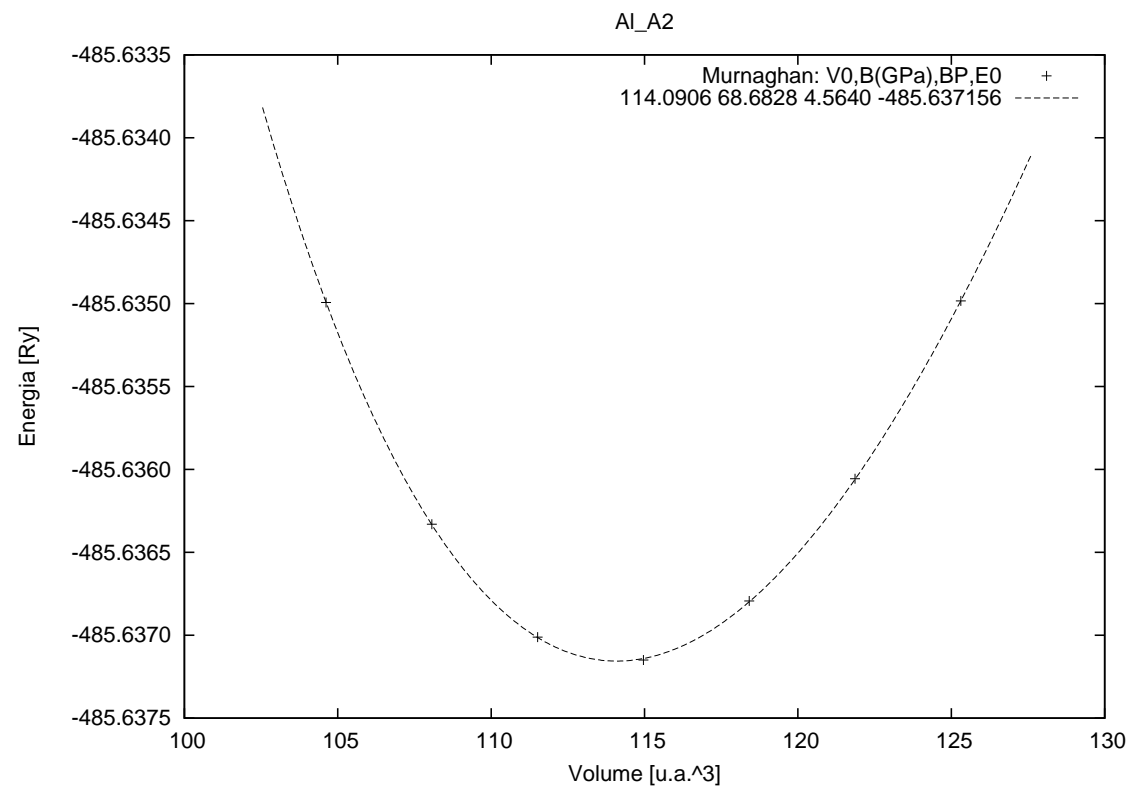

Figura 4.3: Otimização estrutural do Alumínio cúbico de corpo centrado (Al-A2) para o caso FP-LAPW.

\subsection{Escolha do termo de troca e correlação.}

Na literatura existem várias aproximações para descrever o termo de troca e correlação sendo os mais utilizados o LSDA e o GGA nas suas diferentes 


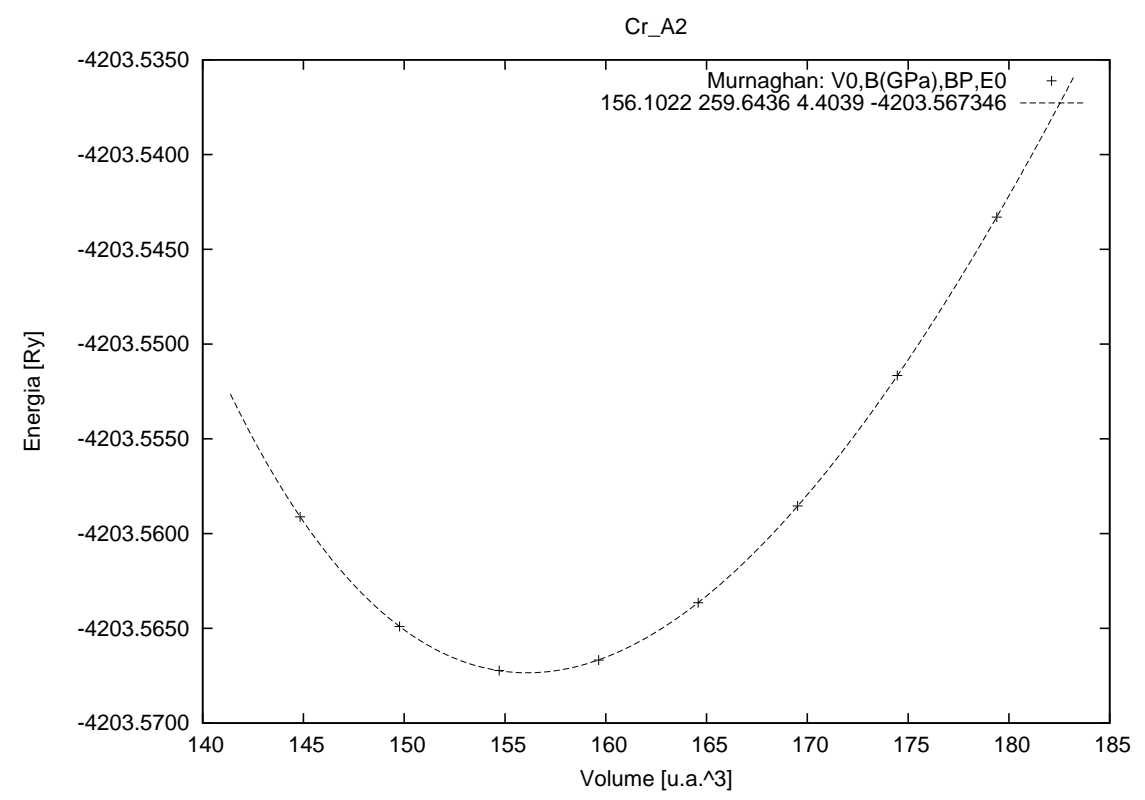

Figura 4.4: Otimização estrutural do Cromo cúbico de corpo centrado (CrA2) para o caso FP-LAPW.

Tabela 4.2: Sistema Unário de referência.

\begin{tabular}{ccccc}
\hline Sistema & Estrutura & $a_{0}$ & MM & $E_{T}$ \\
\hline $\mathrm{Fe}(\mathrm{FM})$ & $\mathrm{A} 2$ & 0.283 & 2.24 & -2545.612324 \\
$\mathrm{Al}(\mathrm{FM})$ & $\mathrm{A} 2$ & 0.323 & 0 & -485.637179 \\
$\mathrm{Cr}(\mathrm{AFM})$ & $\mathrm{A} 2$ & 0.285 & 0 & -2101.784485 \\
\hline
\end{tabular}

parametrizações [35] e [41]. Escolhemos novamente o Fe como sistema teste pelo fato de que este apresenta experimentalmente momento magnético no estado fundamental e é um exemplo bastante estudado na literatura. Observase que utilizando a LSDA e fazendo cálculos sem polarização de spin o estado mais estável do Fe corresponde a estrutura FCC. Quando permitimos a formação de momento magnético no Fe (cálculo com polarização de spin) à energia da fase BCC diminui, porém a estrutura FCC continua sendo a mais estável. Este fato contradiz o resultado experimental, uma vez que a estrutura mais estável do Fe a baixas temperaturas é BCC e magnética [88]. Concluímos assim, de acordo com o já observado na literatura através de diferentes métodos [89] e [90] que a LSDA não reproduz corretamente o estado fundamental do Fe. Por outro lado, observa-se que quando cálculos são realizados com GGA [48] e sem polarização de spin o estado mais estável corresponde á estrutura FCC, de maneira análoga ao resultado obtido 

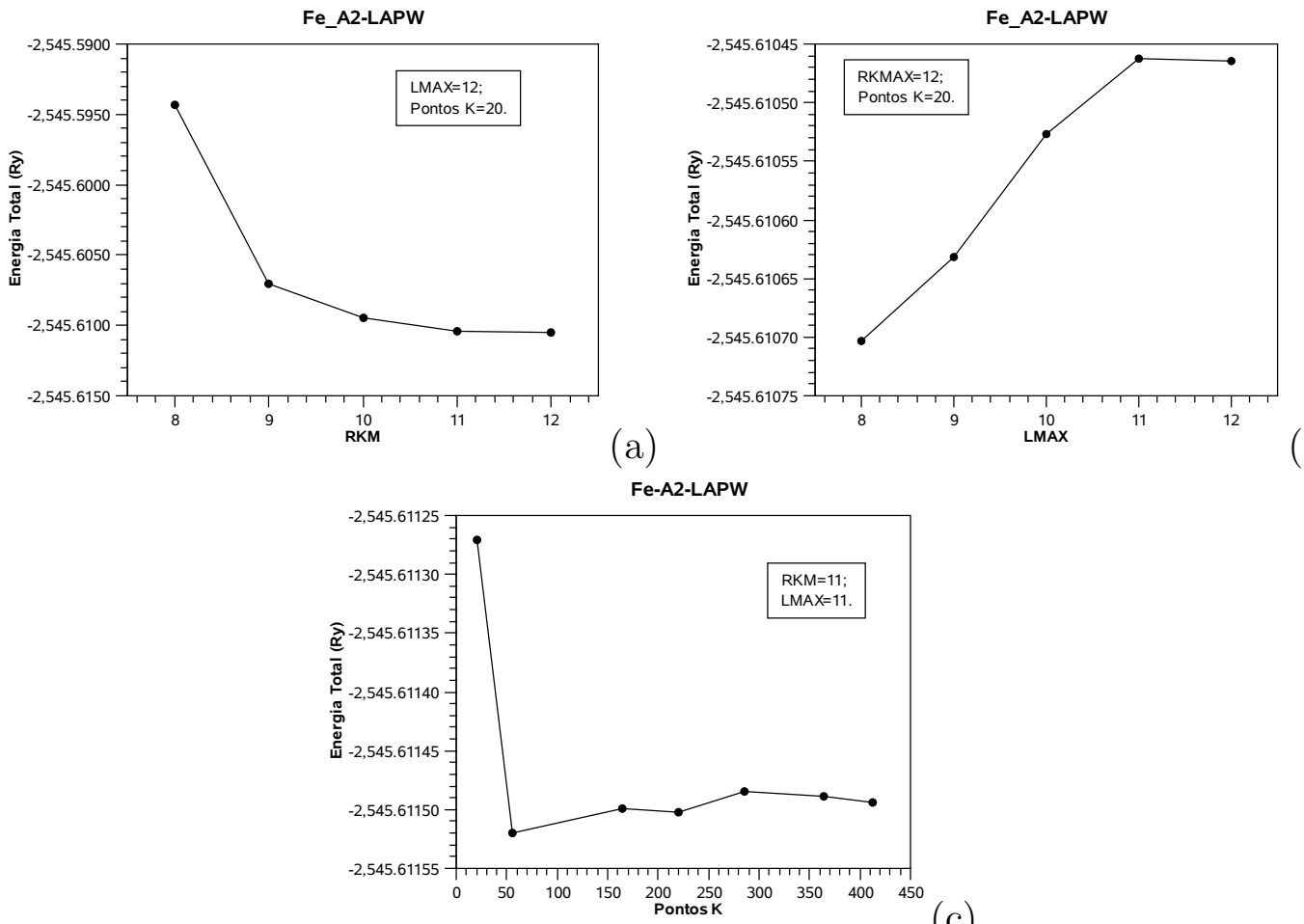

(c)

Figura 4.5: Variação da energia total em função de: (a) RKmax, (b) - Lmax e (c) - dos pontos K na primeira zona irredutível de Brillouin.

com LSDA. Mas, quando permitimos a formação de momento magnético no Fe, obtém-se que a estrutura BCC é a mais estável, o que reproduz o observado experimentalmente. Por este motivo, o funcional GGA [48] foi escolhido para os demais cálculos deste trabalho. 


\section{Capítulo 5}

\section{Resultados e}

\section{Discussões: 02 - As Influências das Premissas do Cálculo Ab-initio no Diagrama de fases}

Os cálculos ab-initio de estrutura eletrônica, no âmbito da Teoria do Funcional da Densidade (DFT), usada como dado de entrada no CVM para o cálculo dos diagrama de fases são agora consideradas viáveis e uma ferramenta poderosa para fornecer informações sobre sistemas para os quais existe uma falta de dados experimentais. Apesar deste recente sucesso, eles dependem de pressupostos básicos ou escolhas para a solução computacional do complicado problema da mecânica quântica de muitos elétrons interagindo. Portanto, o cálculo de diagramas de fase pode depender destas escolhas. É o objetivo do presente capítulo demonstrar a influência de alguns aspectos metodológicos dos cálculos ab-initio realizado com o WIEN2K e os cálculos pseudopotenciais com o VASP no diagrama de fase resultante. Usamos o estado da arte Full Potential-Linear Augmented Plane Wave (FP-LAPW) e o método Projector Augmented Wave (PAW) para executar cálculos de estrutura eletrônica combinado com o Método Variacional de Cluster na aproximação do tetraedro irregular para calcular o diagrama de fases BCC do Fe-Mo. Os resultados apresentados aqui foram submetidos para publicação.[49]

\subsection{Metodologia}

Utilizando o procedimento detalhado no capítulo 03 para o cálculo do diagrama de fases do Mo-Fe. O conjunto de compostos usados para o estudo 
das influências das premissas do estilo de cálculo efetuado no diagrama de fases foram: as estruturas A2 Mo, A2 Fe, B2 MoFe, B32 MoFe, D0 ${ }_{3} \mathrm{Mo}_{3} \mathrm{Fe}$ e $\mathrm{D}_{3} \mathrm{MoFe}_{3}$. Vale lembrar que este conjunto é bastantes limitados em comparação, por exemplo, com o utilizado por Blum e Zunger para investigar o sistema Mo-Ta BCC (52 compostos) [93] numa abordagem alternativa dentro da familia de métodos de expansão de cluster[13]. O conjunto da base é mantido limitado aqui uma vez que apenas uma descrição simplificada da termodinâmica do sistema com base na aproximação do tetraedro irregular do método variacional de cluster (CVM) é o alvo (descrição das fases BCC). As energias totais dos compostos ordenados foram calculados usando o Código WIEN2K. Isso permite testar o efeito de três grupos de pressupostos utilizados no cálculo de estrutura eletrônica, que são:

1. a escolha da base para a expansão da função de onda,

2. a escolha do termo de troca-correlação do potencial elétron-elétron, e

3. a consideração de efeitos relativísticos e acoplamento spin-órbita.

O primeiro conjunto de cálculos, denotado aqui por "FPLAPW + LSDA" foi realizado com a base tradicional usada no método Full Potential Linearized Augmented Plane Wave (FP-LAPW), que é formado por funções de onda como orbitais dentro das esferas e ondas planas na região intersticial. O efeito de troca-correlação aqui considerado usou a mais simples aproximação com a DFT, onde apenas a densidade eletrônica local (spin) é considerada (A chamada aproximação local da densidade de Spin, LSDA)[44]. As correções Relativista só serão aplicadas de forma simplificada, utilizando a implementação escalar-relativista do algoritmo[94].

O segundo conjunto, chamado "FPLAPW + GGA" usa a mesma base que o conjunto anterior, mas agora os efeitos de troca-correlação são calculados usando a chamada "Generalized Gradient Approximation"(GGA). Deve-se ter em mente que, ao contrário do LSDA, a implementação do GGA não é única. No presente trabalhar a implementação sugerida por Perdew, Burke e Ernzernhof (PBE) [48] foi usada. Como no caso anterior, somente correções escalar-relativísticas foram aplicadas para este conjunto de cálculos.

O terceiro conjunto, denominado "FPLAPW + GGA + SO" foi semelhante ao anterior ("FPLAPW + GGA"), mas agora uma correção relativística de segunda ordem é utilizada, considerando-se o acoplamento spinórbita[95].O quarto conjunto, chamado "FPLAPW + GGA(NR)" vai na direção oposta, evitando correções relativísticas. Isto é feito, para investigar se tais correções realmente afetam os resultados. 
Finalmente, o quinto conjunto de cálculos foi realizado utilizando o Projetor Augmentadas Wave (PAW) [96] como método implementado no pacote de simulação "ab-initio Vienna" (VASP código) ${ }^{1}$ usando a mesma aproximação GGA [48] e correções escalar relativista utilizadas no segundo conjunto.

Os parâmetros de rede no estado fundamental foram determinadas através do cálculo do valor mínimo da energia total. Como a energia total é o parâmetro mais importante na presente abordagem, um cuidado especial foi tomados ao realizar testes de convergência. Seguindo a notação WIEN2K a convergência da ordem de $10^{-6}$ Ry na energia total foi alcançada usando valores adequados de $\mathrm{RK}_{\max }, \mathrm{L}_{\max }$ e número de pontos-K, que são parâmetros de controle para a convergência no cálculo FP-LAPW, como discutido no capítulo anterior.

A convergência pode ser alcançada por caminhos diferentes e aqui nós escolhemos primeiro variar $R K_{\max }$, mantendo $\mathrm{L}_{\max }$ e do número de pontos-K fixos, então variar $\mathrm{L}_{\max }$ (mantendo $R K_{\max }$ e número de pontos-K fixos) e, por último, variar o número de pontos-K, com os outros parâmetros fixos. O processo de autoconsistente tem sido feito até que a diferença de energia total por célula unitária seja menor do que $10^{-6} \mathrm{Ry}$ entre iterativos cálculos. No caso dos cálculos com o código VASP a função de onda do método PAW[96], com uma energia cinética de corte de 370 eV uma expansão base de ondas planas foi usada. Convergência na energia total foi alcançada quando se diferem em menos de $1.0 \times 10^{-5} \mathrm{eV}$ entre duas interações autoconsistente. Todos os cálculos foram realizados com spin polarizado. A integração na zona de Brillouin foi empregando uma amostragem de $15 \times 15 \times 15$ pontos-K de mesh no esquema Monkhorst-Pack [97]. Os níveis de energia eletrônica foram ampliadas usando o esquema de Methfessel-Paxton [98] com uma ampliação de $0,1 \mathrm{eV}$. As energias totais calculada para a substância $\Phi(\operatorname{com} \Phi=\mathrm{B} 2$, $\mathrm{B} 32$ ou $\left.\mathrm{D}_{3}\right)$, com estequiometria $M o_{x} F e_{y}$ e $(\mathrm{n}=\mathrm{x}+\mathrm{y}$ átomos na fórmula unidade) são denotados por $U_{M o_{x} F e_{y}}^{\Phi}$, e deve ser convertida em energias de formação, $\Delta^{f} U_{M o_{x} F e_{y}}^{\Phi}$, por mol de átomos no estado de referência da mistura mecânica dos componentes (com estrutura BCC) para a implementação na CVM. Isso é feito utilização

$$
\Delta^{f} U_{M o_{x} F e_{y}}^{\Phi}=\frac{U_{M o_{x} F e_{y}}^{\Phi}-x U_{M o}^{A 2}-y U_{F e}^{A 2}}{n}
$$

Como a estrutura do ferro A2 é conhecida por ser ferromagnético, todos os cálculos são feitos com spin polarizado. As energias de formação calculadas correspondem, portanto, ao composto magneticamente ordenado em alguns

\footnotetext{
${ }^{1}$ Este último conjunto de cálculo foi realizado em colaboração com o Dr. Joelson Cott Garcia da Escola Politécnica da USP.
} 
dos casos. As energias de formação dos quatro compostos são utilizados como parâmetros de entrada no formalismo da mecânica estatística (CVM) adotada para o cálculo do diagrama de fase. O CVM foi primeiramente proposto por Kikuchi, como descrito no capítulo 03, como um método para derivar expressões para a entropia de reticulados arbitrários em aproximações arbitrárias. Mais tarde, foi reconhecido que a CVM fornece um procedimento padrão para obter funções de partições fatoráveis de sistemas de redes com a abordagem de campo -médio.

O conceito central da CVM é o "cluster básico", o qual corresponde a uma figura geométrica contida na rede de referência (não necessariamente restrita a pontos de rede), que representa o intervalo de comprimentos de correlação explicitamente considerados na aproximação. No presente trabalho a aproximação do tetra-irregulares (TI) de cluster para a rede BCC foi empregada. O formalismo CVM na aproximação do cluster de TI tem sido exaustivamente descrito em várias publicações anteriores do grupo e foi descrito em detalhes no capítulo 03 e portanto, apenas os aspectos que são relevantes para a discussão dos resultados serão reproduzidos aqui. A energia interna (U) de uma rede $\mathrm{BCC}$ com sitios $\mathrm{N}$ (contendo $6 \mathrm{~N}$ tetraedros irregulares) é escrita como

$$
U=6 N \sum_{i, j, k, l=F e, M o} \varepsilon_{i, j, k, l}^{\alpha \beta \gamma \delta} \rho_{i, j, k, l}^{\alpha \beta \gamma \delta} .
$$

Nesta expressão, $\rho_{i j k l}^{\alpha \beta \gamma \delta}$ representa a probabilidade de encontrar um $\{\alpha \beta \gamma \delta\}$ cluster TI com configuração $\{i j k l\}$ (ou seja, espécies $i$ ocupando a posição no clusters $\alpha, j$ na posição $\beta$ do cluster e assim por diante...) Entre os tetraedros $6 \mathrm{~N}$ do sistema e $\varepsilon_{i j k l}^{\alpha \beta \gamma \delta}$ representa um autovalor associado com esta configuração. O composto estequiométrico no estado fundamental corresponde ás seguintes configurações: um composto estequiométrico B2 MoFe com pontos de rede $\mathrm{N}$, por exemplo, pode ser construído por $6 \mathrm{~N}$ tetraedros com configuração FeFeMoMo, enquanto o $\mathrm{B} 32 \mathrm{MoFe}, \mathrm{D0}_{3} \mathrm{Mo}_{3} \mathrm{Fe}$ e $\mathrm{MoFe}_{3}$ compostos estequiométricos são igualmente obtidos usando como configurações MoMoMoFe MoFeMoFe e MoFeFeFe. A equação 5.2 pode ser agora aplicada a esses casos particulares e a energia de formação destes compostos estão associadas com os autovalores do cluster correspondente. No caso do composto MoFe B2, por exemplo:

$$
\varepsilon_{M o, M o, F e, F e}^{\alpha \beta \gamma \delta}=\frac{\Delta^{f} U_{M o F e}^{B 2}}{6 N}
$$

lembrando que os autovalores da matriz $\left\{\varepsilon_{i, j, k, l}^{\alpha \beta \gamma \delta}\right\}$ são altamente degenerada pelos vinculos de simetria da rede cristalina. 
Ambas configurações FeMoFeMo e MoFeMoFe, por exemplo, representam um composto estequiométrico MoFe B32, diferindo apenas de uma translação da origem da rede pelo vetor $\frac{a_{0}}{2}[111]$ (onde $a_{0}$ representa a constante de rede da estrutura B32). Desde que a energia do composto não dependa da escolha da origem, segue que $\varepsilon_{M o, F e, M o, F e}^{\alpha \beta \gamma \delta}=\varepsilon_{F e, M o, F e, M o}^{\alpha \beta \gamma \delta}$. O mesmo é verdade para as duas configurações degeneradas restantes FeMoMoFe e MoFeFeMo.

O conjunto $\left\{\varepsilon_{i j k l}^{\alpha \beta \gamma \delta}\right\}$, incluindo os dois valores de referência, $\varepsilon_{M o, M o, M o, M o}^{\alpha \beta \gamma \delta}=$ $\varepsilon_{F e, F e, F e, F e}^{\alpha \beta \gamma \delta}=0$, contendo todas as informações necessárias para a descrição do sistema. É um pratica comum na literatura decompor a configuração de autovalores em contribuição de pares (primeira e segunda vizinhança), completando o conjunto com outras interações do cluster. Não é o que fazemos aqui, uma vez que os resultados são apreciados usando as energias de formação dos compostos.

\subsection{Resultados e discussões das premissas do cálculo}

Na Tabela 5.11 são apresentados os resultados obtidos no cálculo de estrutura eletrônica. Esta tabela lista as energias de coesão, parâmetro de rede de equilíbrio, $a_{0}$, e momentos magnéticos médios, $\mu$, dos seis compostos que formam o base da expansão de cluster para o sistema BCC Mo-Fe utilizando as diferentes aproximações descritas na seção anterior. Os parâmetros de controle para convergência do código WIEN2K para os cálculos de estrutura eletrônica não foram muito sensíveis á escolha do conjunto metodológico. A condição $\mathrm{RK}_{\max }=\mathrm{L}_{\max }$ foi obtido na convergência de todos os compostos (em todos os conjuntos), a convergência foi alcançada com $\mathrm{RK}_{\max }=10$, onde os raios de muffin-tin $\mathrm{R}=2.0$ u.a. para todas as espécies atômicas foram usadas, e 413 pontos-K. A energia máxima $\left(\mathrm{E}_{\max }\right)$ da janela de energia no caso "FPLAPW + SO + GGA" durante o cálculo com o WIEN2K foi aumentada para 10 Ry e um adicional da função de onda para parte radial $\mathrm{p} \frac{1}{2}$ foi adicionado neste caso.

Com relação ao parâmetro de rede de equilíbrio e o momento magnético, observamos que as maiores variações são observadas entre o conjunto LSDA enm relação aos conjuntos feitos com a aproximação GGA. Este efeito já havia sido descrito na literatura por K. Capelle no artigo [41]. Deve se ter em mente que somente os elementos puros podem ser observados experimentalmente. Os valores experimentais para o parâmetro de rede em equilíbrio na temperatura ambiente são $0.2867 \mathrm{~nm}$ para o Fe A2 e $0.32470 \mathrm{~nm}$ para o Mo A2 [99], que concorda muito bem com os valores apresentados na Tabela 5.1 
Tabela 5.1: Energia Total volumetrica, $U_{M o_{x} F e_{y}}^{\Phi}$, calculada para os seis compostos formando a base para a expansão de cluster aplicada ao sistema BCC Mo-Fe usando os cinco conjuntos de premissas descrito no texto.O Valor do momento magnético médio na celula unitária, $\mu$, são também apresentados( em Magneton de Bohr, $\left.\mu_{B}\right)$. Parâmetros de rede de equilíbrio são expressos em nm e os valores da energia são representados em Rydberg (Ry) por formula unitária $\left(1 \mathrm{Ry}=2.1798741 \times 10^{-18} \mathrm{~J}\right)$

\begin{tabular}{|c|c|c|c|c|c|c|}
\hline Cálculo & Estequiometria & Estrutura & $\overline{a_{0}}$ & $\mu$ & $\overline{\mathrm{B}(\mathrm{GPa})}$ & $\overline{U_{M o_{x} F e_{y}}^{\phi}}$ \\
\hline \multirow[t]{6}{*}{$\overline{\text { FPLAPW+LSDA }}$} & $\mathrm{Fe}$ & $\overline{\mathrm{A} 2}$ & 0.276 & 2.03 & 247 & -2541.169272 \\
\hline & Mo & A2 & 0.312 & 0 & 291 & -8090.871113 \\
\hline & $\mathrm{MoFe}$ & B2 & 0.295 & 1.58 & 268 & -10632.015100 \\
\hline & $\mathrm{MoFe}$ & B32 & 0.592 & 1.76 & 261 & -21264.033962 \\
\hline & $\mathrm{MoFe}_{3}$ & $\mathrm{D}_{3}$ & 0.570 & 1.90 & 267 & -15714.368255 \\
\hline & $\mathrm{Mo}_{3} \mathrm{Fe}$ & $\mathrm{D}_{3}$ & 0.608 & 1.76 & 273 & -26813.739400 \\
\hline \multirow[t]{6}{*}{ FPLAPW+GGA } & $\mathrm{Fe}$ & $\mathrm{A} 2$ & 0.284 & 2.22 & 181 & -2545.612648 \\
\hline & Mo & $\mathrm{A} 2$ & 0.316 & 0 & 260 & -8099.182722 \\
\hline & $\mathrm{MoFe}$ & B2 & 0.301 & 2.03 & 217 & -10644.770238 \\
\hline & $\mathrm{MoFe}$ & B32 & 0.603 & 2.08 & 215 & -21289.541287 \\
\hline & $\mathrm{MoFe}_{3}$ & $\mathrm{DO}_{3}$ & 0.584 & 2.22 & 198 & -15736.004548 \\
\hline & $\mathrm{Mo}_{3} \mathrm{Fe}$ & $\mathrm{D}_{3}$ & 0.618 & 2.17 & 232 & -26843.118678 \\
\hline \multirow[t]{6}{*}{ FPLAPW+GGA+SO } & $\mathrm{Fe}$ & A2 & 0.284 & 2.24 & 178 & -2545.614418 \\
\hline & Mo & $\mathrm{A} 2$ & 0.316 & 0 & 247 & -8099.207279 \\
\hline & $\mathrm{MoFe}$ & $\mathrm{B} 2$ & 0.301 & 2.03 & 225 & -10644.796517 \\
\hline & $\mathrm{MoFe}$ & B32 & 0.603 & 2.08 & 211 & -21289.593637 \\
\hline & $\mathrm{MoFe}_{3}$ & $\mathrm{D}_{3}$ & 0.584 & 2.21 & 198 & -15736.034425 \\
\hline & $\mathrm{Mo}_{3} \mathrm{Fe}$ & $\mathrm{DO}_{3}$ & 0.618 & 2.17 & 235 & -26843.193755 \\
\hline \multirow[t]{6}{*}{ FPLAPW+GGA(NR) } & $\mathrm{Fe}$ & A2 & 0.286 & 2.31 & 170 & -2527.264046 \\
\hline & Mo & A2 & 0.318 & 0 & 242 & -7954.688782 \\
\hline & $\mathrm{MoFe}$ & B2 & 0.303 & 2.09 & 197 & -10481.927536 \\
\hline & $\mathrm{MoFe}$ & B32 & 0.607 & 2.13 & 196 & -20963.858425 \\
\hline & $\mathrm{MoFe}_{3}$ & $\mathrm{D}_{3}$ & 0.583 & 2.20 & 180 & -15536.460651 \\
\hline & $\mathrm{Mo}_{3} \mathrm{Fe}$ & $\mathrm{DO}_{3}$ & 0.617 & 2.11 & 214 & -26391.289895 \\
\hline \multirow[t]{6}{*}{$\overline{\mathrm{VASP}(\mathrm{GGA})}$} & $\mathrm{Fe}$ & $\mathrm{A} 2$ & 0.284 & 2.21 & 176 & -0.610963 \\
\hline & Mo & $\mathrm{A} 2$ & 0.315 & 0 & 268 & -0.804478 \\
\hline & $\mathrm{MoFe}$ & B2 & 0.300 & 1.98 & 217 & -1.388647 \\
\hline & $\mathrm{MoFe}$ & B32 & 0.601 & 2.02 & 216 & -2.782464 \\
\hline & $\mathrm{MoFe}_{3}$ & $\mathrm{DO}_{3}$ & 0.583 & 1.93 & 200 & -2.619679 \\
\hline & $\mathrm{Mo}_{3} \mathrm{Fe}$ & $\mathrm{DO}_{3}$ & 0.617 & 2.03 & 235 & -2.983457 \\
\hline
\end{tabular}

para os conjuntos que usou a aproximação GGA. O mesmo é verdadeiro para o valor reportado do momento magnético de equilíbrio médio do Fe A2 ( $\mu=$ 
$2.216 \mu_{B}$ ) [99]. Referente ao bulk modulus, os valores experimentais para ambos Fe e Mo pode ser obtido pelo uso de dados policristais (respectivamente 169,8 e 261,2 GPa) ou simplesmente usando a constante elastica do cristal simples (respectivamente 167 e $265 \mathrm{GPa}$ ) [99]. Como esperado, o valor calculado para o Mo está em melhor concordância no caso onde ambas as correções relativisticas e o GGA são usados. A comparação para o Fe mostra uma leve melhor concordância no caso dos calculos com o VASP, mas deve ser lembrado que os valores calculados referem-se ao estado fundamental ( $\mathrm{T}=0 \mathrm{~K})$, enquanto os dados experimentais são usualmente tomados a temperatura ambiente, e a constante elastica usualmente mostra uma pequena discrepância com o aumento da temperatura[100], [101]. A tabela 5.2 mostra a energia de formação calculada de todos os compostos no estado de referência da mistura mecânica de Mo BCC (A2) e Fe BCC (A2) nos cinco conjuntos de de calculos. Os resultados da tabela 5.2 são mostrados graficamente na figura 5.1

Tabela 5.2: Energia de Formação dos cinco conjuntos calculados (kJ mol $\left.{ }^{-1}\right)$.

\begin{tabular}{|c|c|c|c|}
\hline Cálculo & Estequiometria & Estrutura & $\Delta^{f} U_{M o_{x} F e_{y}}^{\Phi}$ \\
\hline \multirow[t]{4}{*}{$\overline{\text { FPLAPW+LSDA }}$} & $\overline{\mathrm{MoFe}}$ & $\overline{\mathrm{B} 2}$ & +16.60 \\
\hline & $\mathrm{Mo}_{3} \mathrm{Fe}$ & $\mathrm{D}_{3}$ & +14.18 \\
\hline & $\mathrm{MoFe}$ & B32 & +15.36 \\
\hline & $\mathrm{MoFe}_{3}$ & $\mathrm{D}_{3}$ & +3.50 \\
\hline \multirow[t]{4}{*}{ FPLAPW+GGA } & $\mathrm{MoFe}$ & $\overline{\mathrm{B} 2}$ & +16.50 \\
\hline & $\mathrm{Mo}_{3} \mathrm{Fe}$ & $\mathrm{D}_{3}$ & +13.83 \\
\hline & $\mathrm{MoFe}$ & B32 & +16.23 \\
\hline & $\mathrm{MoFe}_{3}$ & $\mathrm{D}_{3}$ & +5.29 \\
\hline \multirow[t]{4}{*}{ FPLAPW+GGA+SO } & $\mathrm{MoFe}$ & $\overline{\mathrm{B} 2}$ & +16.53 \\
\hline & $\mathrm{Mo}_{3} \mathrm{Fe}$ & $\mathrm{D}_{3}$ & +13.95 \\
\hline & MoFe & B32 & +16.33 \\
\hline & $\mathrm{MoFe}_{3}$ & $\mathrm{D}_{3}$ & +5.29 \\
\hline \multirow[t]{4}{*}{ FPLAPW+GGA(NR) } & $\mathrm{MoFe}$ & B2 & +16.60 \\
\hline & $\mathrm{Mo}_{3} \mathrm{Fe}$ & $\mathrm{D}_{3}$ & +13.29 \\
\hline & MoFe & B32 & +15.50 \\
\hline & $\mathrm{MoFe}_{3}$ & $\mathrm{D}_{3}$ & +6.65 \\
\hline \multirow[t]{4}{*}{$\operatorname{VASP}(G G A)$} & $\mathrm{MoFe}$ & $\overline{\mathrm{B} 2}$ & +17.59 \\
\hline & $\mathrm{Mo}_{3} \mathrm{Fe}$ & $\mathrm{D}_{3}$ & +13.44 \\
\hline & MoFe & B32 & +15.89 \\
\hline & $\mathrm{MoFe}_{3}$ & $\mathrm{D}_{3}$ & +5.81 \\
\hline
\end{tabular}

Esta forma de apresentação foi escolhida de modo a permitir uma melhor visualização das diferenças entre cada conjunto. A figura 5.1 mostra que algumas das características qualitativas da energia de formação destes compostos 
não dependem do tipo de conjunto calculado. Por exemplo, todas as energias de formação são positivas e também a sequência de estabilidade é a mesma em todos os casos: MoFe - B2 e MoFe - B32 os maiores valores da energia de formação (ou seja, mais positivos), enquanto as energias de formação dos compostos $\mathrm{Mo}_{3} \mathrm{Fe}-\mathrm{D0}_{3}$ apesar de ser menor que as B2 e B32, é maior do que a energia de formação do $\mathrm{MoFe}_{3}-\mathrm{D}_{3}$. A figura 5.1, entretanto, mostra que a variações da ordem de $\pm=1 \mathrm{~kJ} \mathrm{~mol}^{-1}$ na energia de formação de um dado composto são observados para todos os compostos dependendo das premissas básicas usadas no cálculo de estrutura eletrônica. Curiosamente está precissão é da mesma ordem de magnitude da menor estimativa de erro nas técnicas padrões de calorimetria [102]

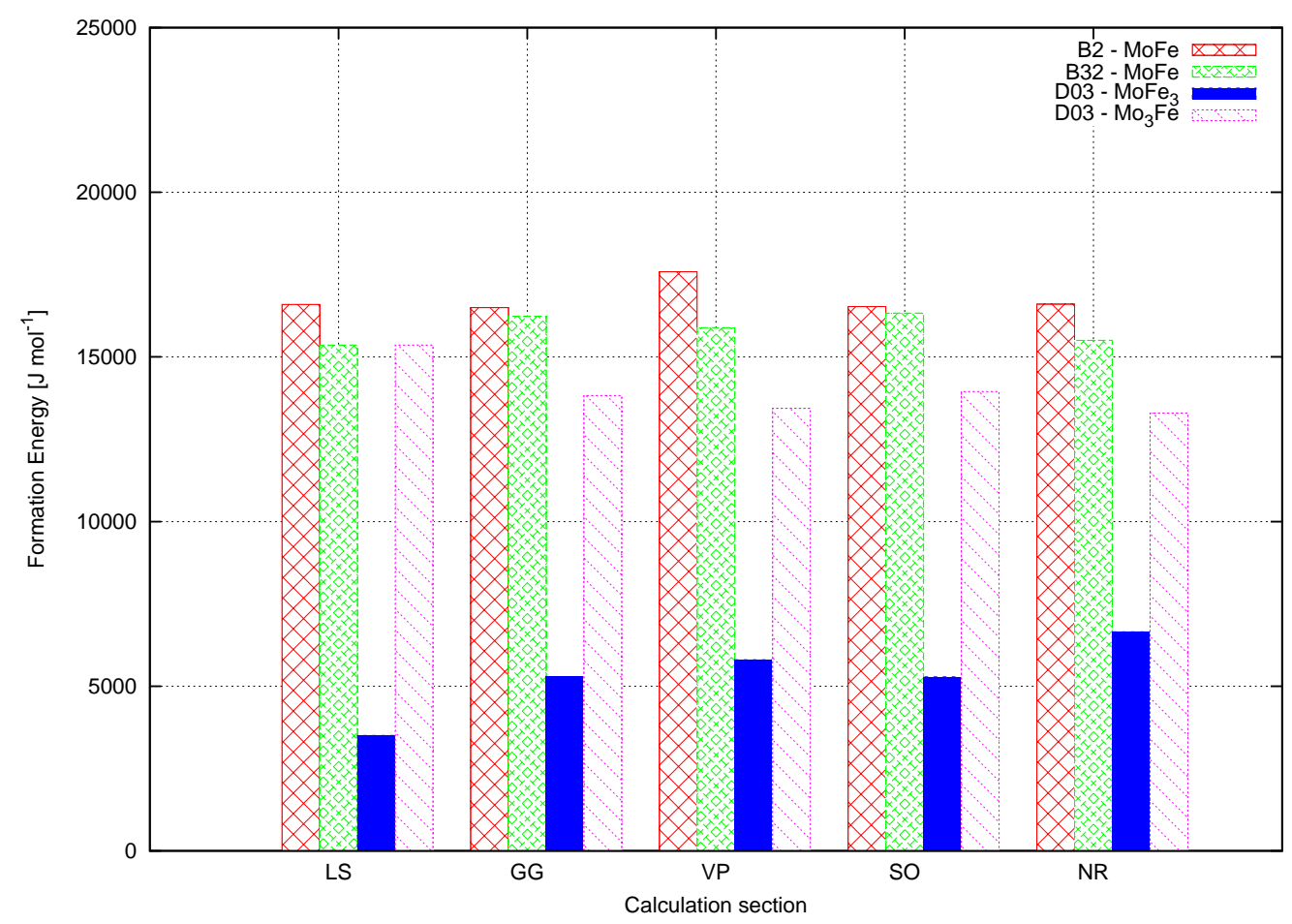

Figura 5.1: Energia de formação dos compostos usados para construir as bases para a expansão de cluster do sistema Mo-Fe de acordo com os cinco conjuntos descritos no texto: $\mathrm{LS}=$ FPLAPW + LSDA, GG = $\mathrm{FPLAPW}+\mathrm{GGA}, \mathrm{SO}=\mathrm{FPLAPW}+\mathrm{GGA}+\mathrm{SO}, \mathrm{NR}=\mathrm{FPLAPW}+\mathrm{GGA}(\mathrm{NR})$ e $\mathrm{VP}=\operatorname{VASP}(\mathrm{GGA})$.

A figura 5.2 mostra os diagramas de fases calculados para as premissas assumidas neste capítulo. Vemos que todas as versões mostram as mesmas 
características qualitativas: um gap de missibilidade assimétrico.
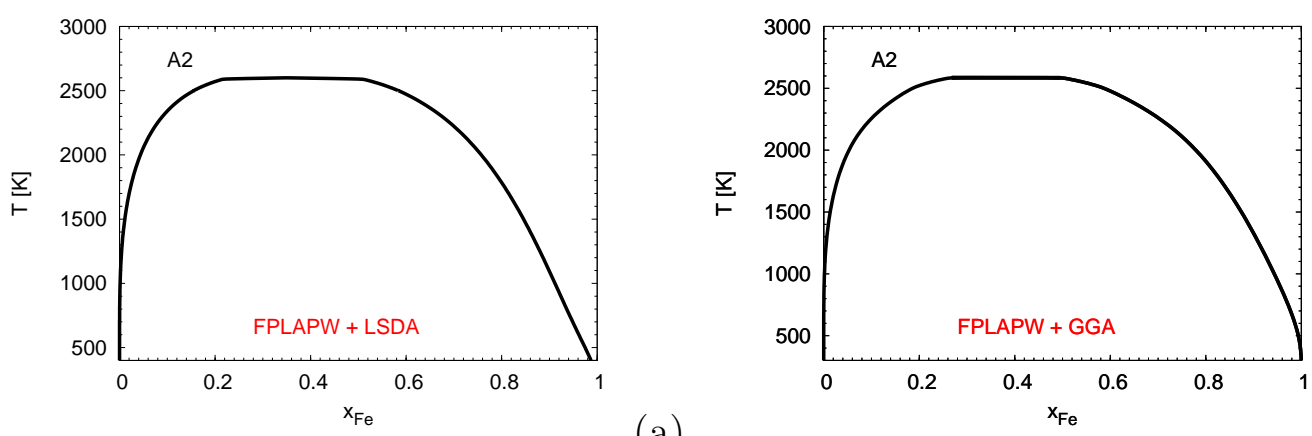

(a)

(b)
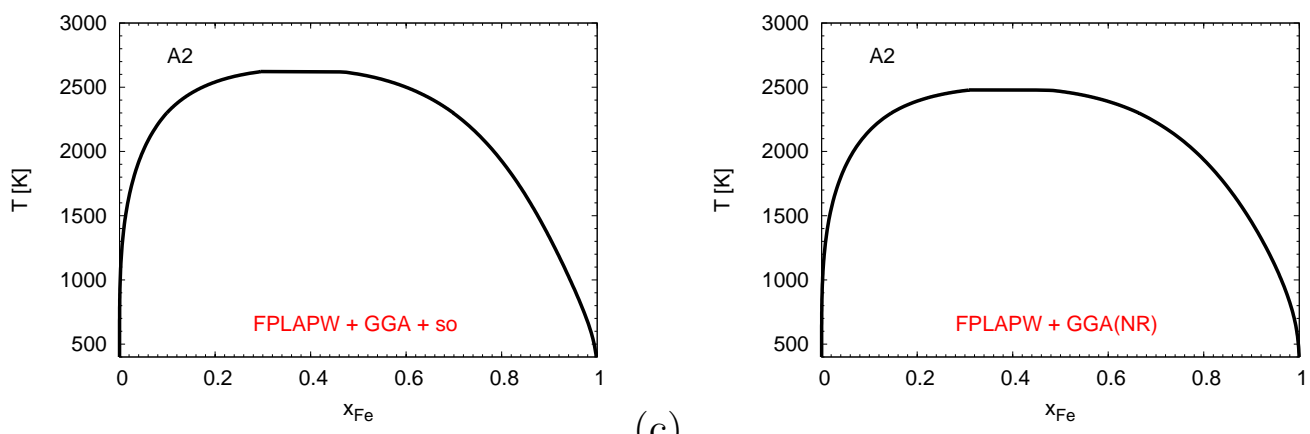

(d)

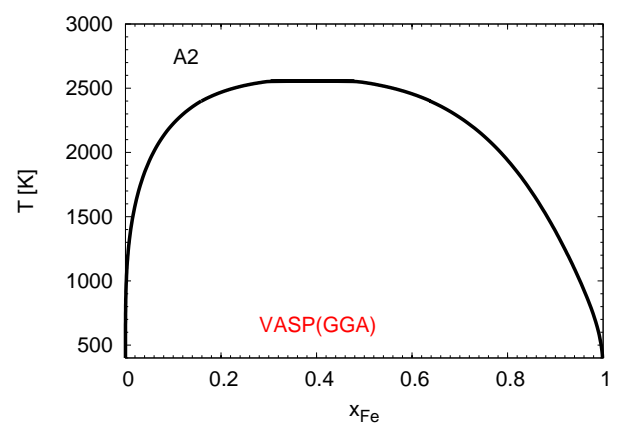

(e)

Figura 5.2: Diagrama de fases Mo-Fe BCC calculado usando as premissas básicas descritas neste capítulo: (a) caso FPLAPW+LSDA, (b) caso FPLAPW+GGA, (c) caso FPLAPW+GGA+SO, (d) caso FPLAPW+GGA(NR) e (e) -caso $\operatorname{VASP}(\mathrm{GGA})$. 


\section{Capítulo 6}

\section{Resultados e \\ Discussões: 03 - Sistemas Binários}

\subsection{Sistemas FeAl}

Ligas do sistema Fe-Al baseadas no reticulado CCC têm atraído grande atenção da comunidade científica e de engenharia de materiais tanto do ponto de vista acadêmico quanto tecnológico. O interesse acadêmico deve-se à existência de ordem configuracional no sistema, sendo que o mesmo pode ser considerado como protótipo para as transições de fases tipo ordem - desordem em reticulados CCC [103]. Do ponto de vista tecnológico, as ligas $\mathrm{CCC}$ do sistema $\mathrm{Fe}-\mathrm{Al}$, na forma de intermetálicos ordenados ou simplesmente no estado desordenado, apresentam-se como potenciais candidatos a materiais para aplicações estruturais a altas temperaturas, devido á sua boa resistência à corrosão e à oxidação, boa resistência mecânica, boa relação resistência mecânica/densidade e custos de produção relativamente baixos [104]. As aplicações tecnológicas, entretanto, ainda são limitadas devido à baixa tenacidade do material à temperatura ambiente [104], [105]. Ligas do sistema $\mathrm{Fe}-\mathrm{Al}$ com até $50 \%$ at. de alumínio apresentam-se em três possíveis estruturas à temperatura ambiente: desordenada CCC (A2), ordenada tipo $\mathrm{FeAl}(\mathrm{B} 2)$ e ordenada tipo $\mathrm{Fe}_{3} \mathrm{Al}\left(\mathrm{D0}_{3}\right)$, ligas ternárias podem ainda apresentar a estrutura da fase de Heusler, tipo $\mathrm{Cu}_{2} \mathrm{MnAl}\left(\mathrm{L} 2_{1}\right)$. A transição entre as diferentes fases ordenadas com o aumento da temperatura ocorre tanto por meio de transições de fases de primeira quanto de segunda ordem [103]. A excelente resistência à corrosão e à oxidação destes materiais se deve à elevada concentração de alumínio na liga, que promove a formação de uma camada 
passiva de $\mathrm{Al}_{2} \mathrm{O}_{3}$ em sua superfície. Devido à alta entalpia de formação deste óxido, esta camada permanece estável mesmo a temperaturas onde o óxido de cromo $\left(\mathrm{Cr}_{2} \mathrm{O}_{3}\right)$ sofre decomposição [106]. Estas propriedades tornam os aluminetos de ferro particularmente atrativos para aplicações a temperaturas moderadas e altas, onde concorrem com os aços inoxidáveis e aços resistentes ao calor ligados com cromo [106]. A excelente resistência à corrosão e à oxidação destes materiais se deve à elevada concentração de alumínio na liga, que promove a formação de uma camada passiva de $\mathrm{Al}_{2} \mathrm{O}_{3}$ em sua superfície. Devido à alta entalpia de formação deste óxido, esta camada permanece estável mesmo a temperaturas onde o óxido de cromo $\left(\mathrm{Cr}_{2} \mathrm{O}_{3}\right)$ sofre decomposição [106]. Estas propriedades tornam os aluminetos de ferro particularmente atrativos para aplicações a temperaturas moderadas e altas, onde concorrem com os aços inoxidáveis e aços resistentes ao calor ligados com cromo [106].

O sistema Fe-Al é considerado um sistema protótipo para estudar o ordenamento de ligas do tipo BCC assim como as interações entre ordem atômica (configuracional) e magnética em ligas. É bastante investigado tanto por abordagens experimentais como teóricas. Na referência [1] foi realizado de forma preliminar o estudo da liga $\mathrm{Fe}-\mathrm{Al}$ utilizando cálculos não relativísticos com e sem spin, sem a inclusão de efeitos relativisticos. Apresentaremos aqui o estudo da liga Fe-Al para o caso em que os cálculos de estrutura eletrônica são relativísticos. Analisaremos a influência no diagrama de fases desta abordagem em relação a realizada para o caso não relativistico. As propriedades locais serão apresentadas no capítulo 08. Calculamos a estrutura eletrônica e determinamos a energia total para os compostos FeAl (nas fases $\mathrm{B} 2$ e $\mathrm{B} 32$ ), $\mathrm{Fe}_{3} \mathrm{Al}$ e $\mathrm{FeAl}_{3}$ (na fase $\mathrm{D0}_{3}$ ) utilizando o código WIEN2K no método FP-LAPW. Estes compostos têm estrutura do tipo BCC (ou são super-reticulados obtidos por ordenação a partir da estrutura BCC), exemplos para estas estruturas bem como os dados cristalográficos utilizados para estes compostos foram mostradas nas figuras do capítulo anterior.

Os resultados da $E_{T}$ dos compostos do sistema Fe-Al com polarização de spin são apresentados na Tabela 6.1.

Também mostramos os valores dos parâmetros utilizados quando o cálculo atinge a convergência dentro do código WIEN2K do método FP-LAPW. Com estes resultados de $E_{T}$ calculamos as energias de formação. A comparação entre valores da energia de formação e dados da literatura exige que ambos sejam expressos em um mesmo referencial termodinâmico. O referencial comumente usado nos cálculos de diagramas é a chamada "mistura mecânica dos componentes".

Na Tabela 6.2 apresentamos os resultados que obtivemos para as energias de formação, por átomo, em unidades de Rydberg (Ry), quilo joule por mol $(k J / m o l)$ e Kelvin vezes constante de Boltzmann $\left(K_{B} K\right)$, utilizando a equiv- 


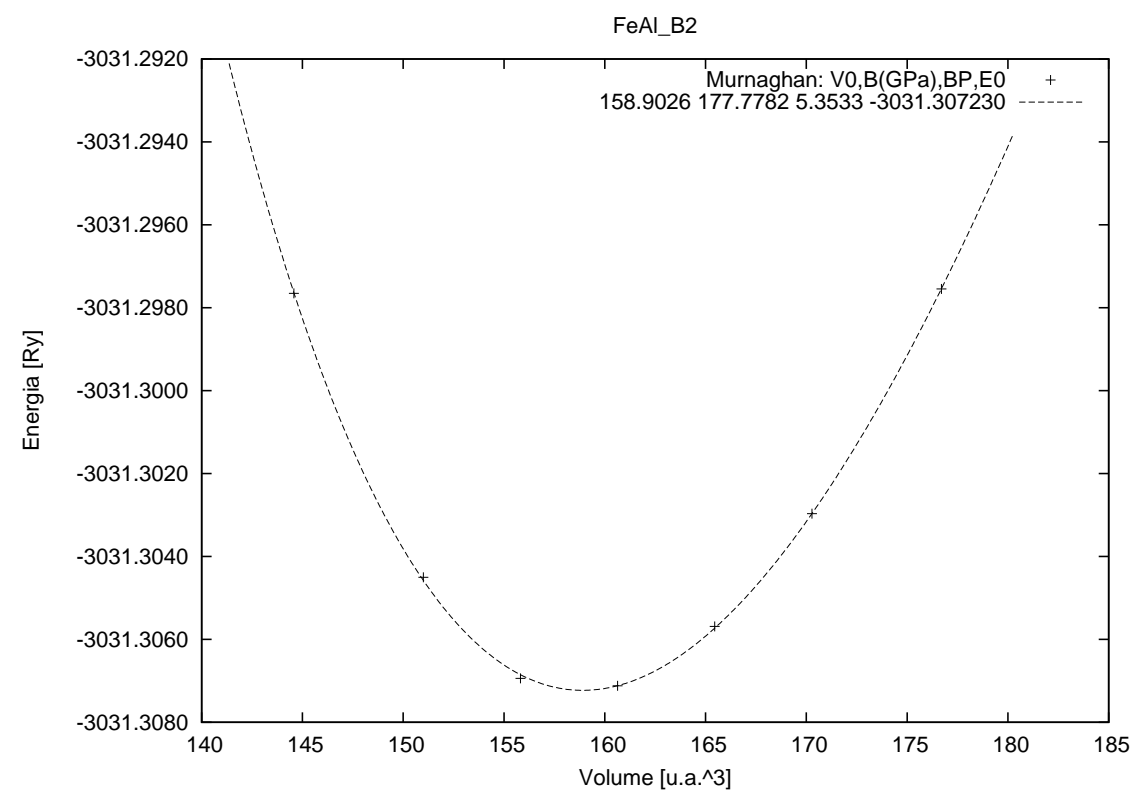

Figura 6.1: Otimização estrutural da liga binária Ferro-Alumínio (FeAl-B2) para o caso FP-LAPW. Na legenda temos: $V_{0}$ é o volume mínimo em unidades atômicas; $\mathrm{B}(\mathrm{GPa})$ é o Bulk módulo, BP é o Derivada do Bulk Módulo e $E_{0}$ a energia mínima.

Tabela 6.1: Energia total calculada para os quatro compostos formando a base para a expansão de cluster aplicada ao sistema Fe-Al. Para todos os calculos Rkmax=Lmax=10, número de pontos $\mathrm{K}$ na zona irredutível de Brillouin foi de 286. O momento magnético médio no átomo de ferro (MM) é apresentado (em magneton de Bohr, $\mu_{B}$ ). Os parâmetros de rede são expressados em nm e os valores da das energias são representados em Rydberg (Ry) por formula unitária $\left(1 \mathrm{Ry}=2.1798741 \times 10^{-18} \mathrm{~J}\right)$.

\begin{tabular}{clcccc}
\hline Calculo & Estequiometria & Estrutura & $a_{0}$ & $\mathrm{MM}$ & $E_{T}$ \\
\hline Ferromagnético & $\mathrm{FeAl}$ & $\mathrm{B} 2$ & 0.295 & 0.72 & -3031.303428 \\
& $\mathrm{FeAl}$ & $\mathrm{B} 32$ & 0.592 & 2.00 & -6062.571664 \\
& $\mathrm{Fe}_{3} \mathrm{Al}$ & $\mathrm{D0}_{3}$ & 0.570 & 2.14 & -8122.539046 \\
& $\mathrm{FeAl}_{3}$ & $\mathrm{D0}_{3}$ & 0.608 & $2.10^{-5}$ & -4002.548986 \\
\hline
\end{tabular}




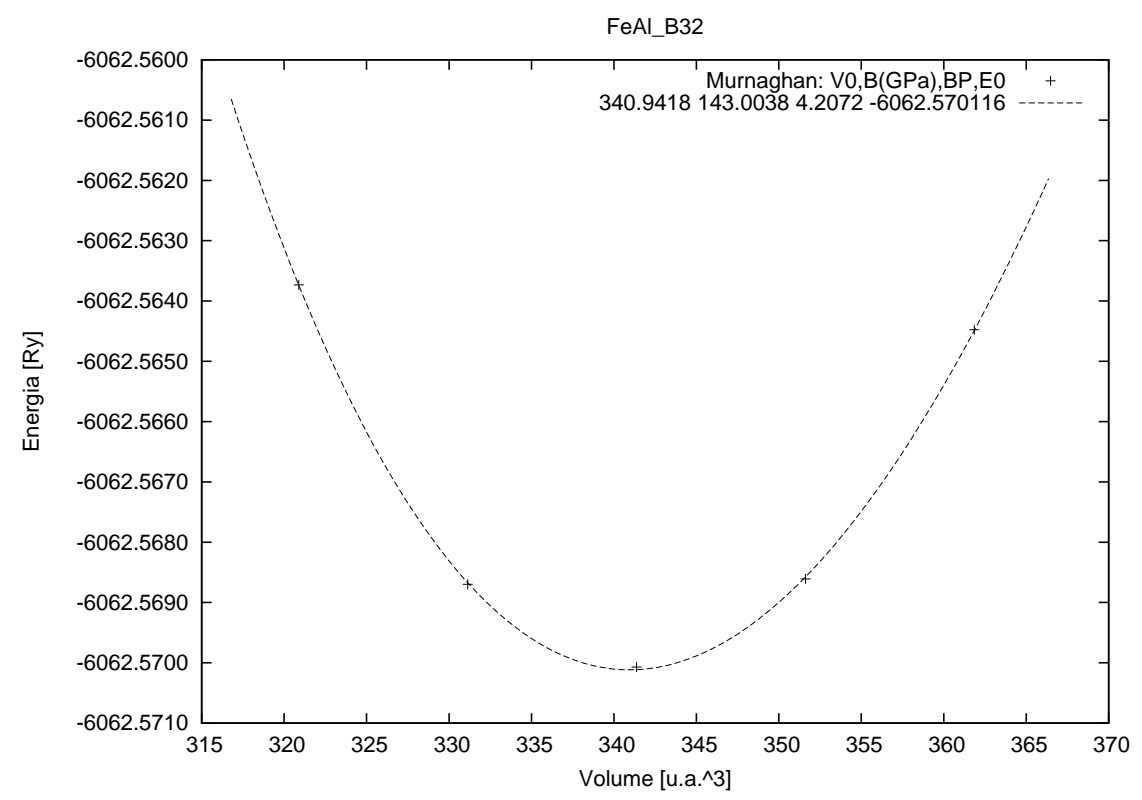

Figura 6.2: Otimização estrutural da liga binária Ferro-Alumínio (FeAl-B32) para o caso FP-LAPW. Na legenda temos: $V_{0}$ é o volume mínimo em unidades atômicas; $\mathrm{B}(\mathrm{GPa})$ é o Bulk módulo, BP é o Derivada do Bulk Módulo e $E_{0}$ a energia mínima.

alência de unidades mostradas abaixo na mesma tabela; por ser um trabalho interdisciplinar, para fácil referência, expressamos a energia de formação nestas três diferentes unidades.

Na Tabela 6.3, estão apresentados os parâmetros de entrada para o formalismo CVM, em unidades de $K_{B} K$. Estes dados foram obtidos usando os dados da Tabela $6.2 \mathrm{com}$ as expressões do capítulo 3, seção (3-3-4). O procedimento é o seguinte:

- a energia de formação da fase B2 (Fe-Al), em unidades de $K_{B} K$, é igualada á expressão (III-39-A) de onde vamos obter o valor do parâmetro de interação $w_{A B}^{(1)}$;

- o valor da energia de formação do $\mathrm{Fe}_{3} \mathrm{Al}$ é igualada á expressão (III39-B) para obter $w_{A B}^{(2)}$ utilizando-se o valor de $w_{A B}^{(1)}$ determinado anteriormente;

- de forma análoga, a energia de formação de B32 (Fe-Al) é igualada á expressão (III-39-C) para obter o $w_{A B A B}$ e o mesmo fazendo com $F e A l_{3}$ e a expressão (III-39-D) para obter $w_{A B B B}$. 


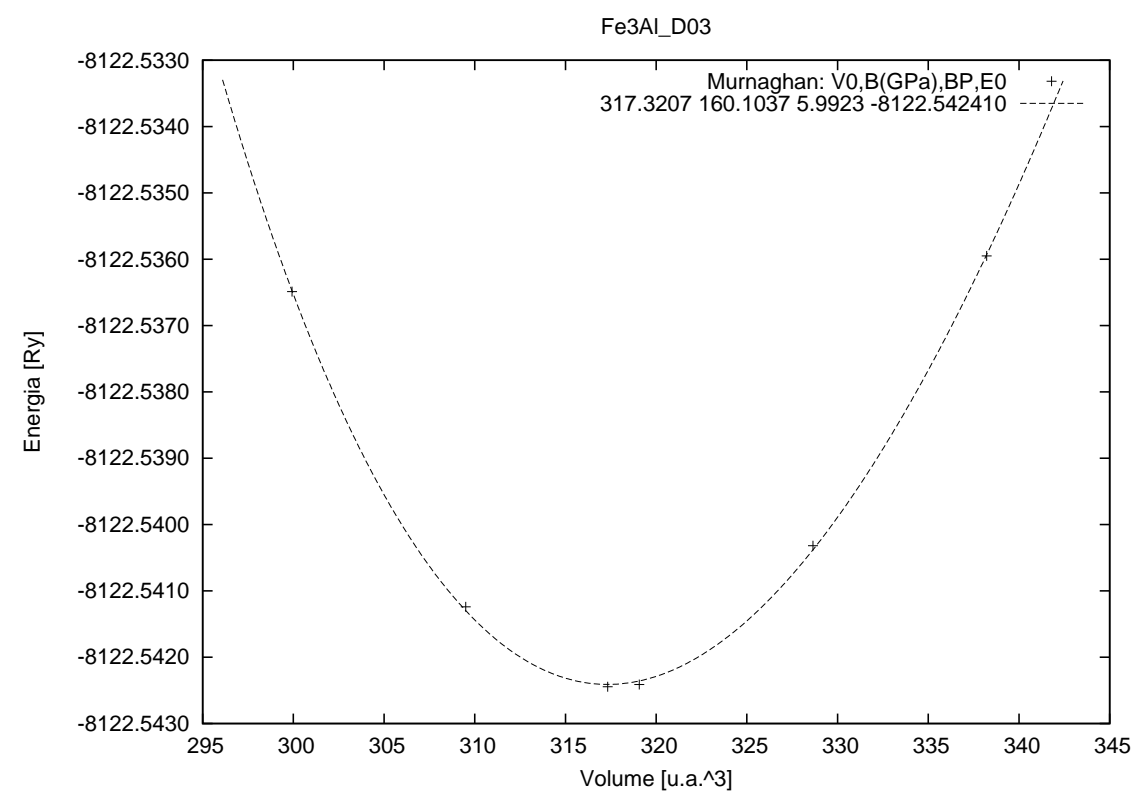

Figura 6.3: Otimização estrutural da liga binária Ferro-Alumínio (Fe3AlD03) para o caso FP-LAPW. Na legenda temos: $V_{0}$ é o volume mínimo em unidades atômicas; $\mathrm{B}(\mathrm{GPa})$ é o Bulk módulo, BP é o Derivada do Bulk Módulo e $E_{0}$ a energia mínima.

\begin{tabular}{ccccc}
\hline Sistema & Fase & Ry & $\mathrm{kJ} / \mathrm{mol}$ & $K_{B} K$ \\
\hline \hline $\mathrm{FeAl}$ & $\mathrm{B} 2$ & -0.0269 & -35.397 & -4257.182 \\
$\mathrm{FeAl}$ & $\mathrm{B} 32$ & -0.0182 & -23.847 & -2868.027 \\
$\mathrm{Fe}_{3} \mathrm{Al}$ & $\mathrm{D} 03$ & -0.0162 & -21.298 & -2561.566 \\
$\mathrm{FeAl}_{3}$ & $\mathrm{D} 03$ & -0.0063 & -8.245 & -991.728 \\
\hline
\end{tabular}

Tabela 6.2: Energia de formação do sistema Fe-Al ferromagnético com polarização de spin e sem acoplamento spin orbita, utilizando o método FP$L A P W$. 


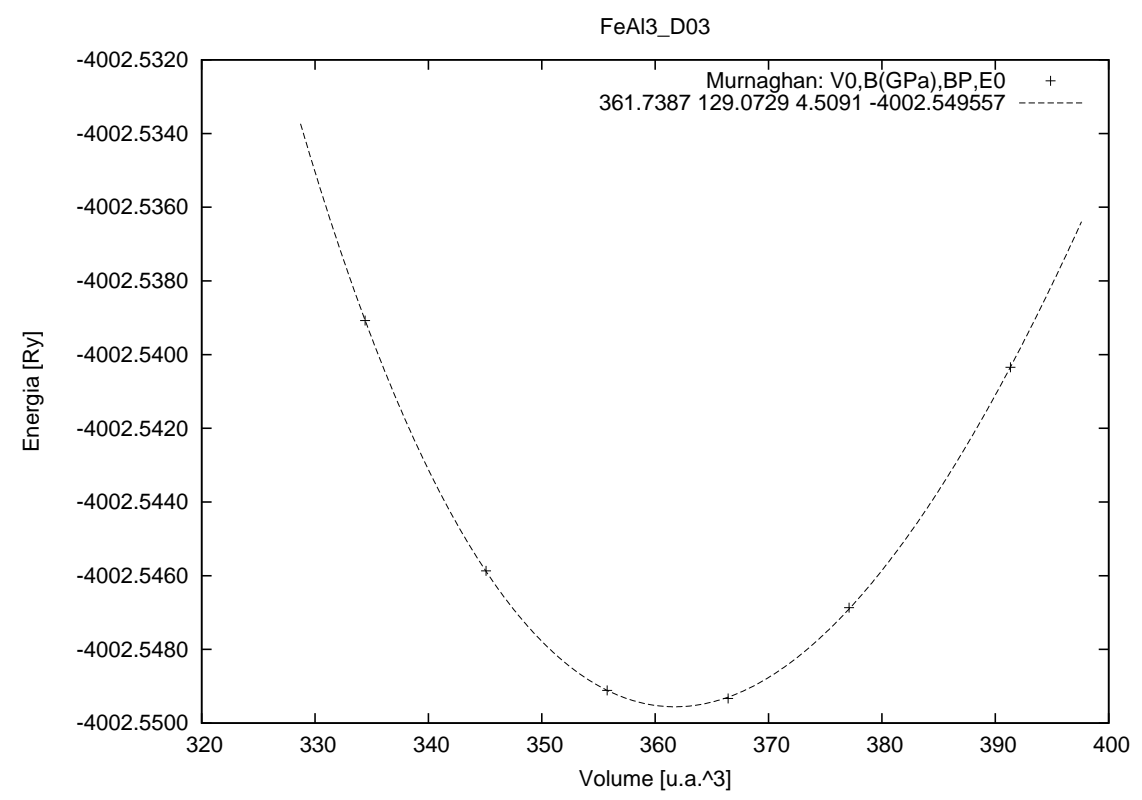

Figura 6.4: Otimização estrutural da liga binária Ferro-Alumínio (FeAl3D03) para o caso FP-LAPW. Na legenda temos: $V_{0}$ é o volume mínimo em unidades atômicas; $\mathrm{B}(\mathrm{GPa})$ é o Bulk módulo, BP é o Derivada do Bulk Módulo e $E_{0}$ a energia mínima.

Na Tabela 6.3 pode-se observar que os valores de $w_{A B}^{(1)}, w_{A B A B}$ e $w_{A B B B}$ tem sinal negativo, o que significa que eles contribuem para a estabilização das fases ordenadas, observa-se também que $W_{A B}^{(2)}$, pelo sinal positivo que tem, contribui, parcialmente, para a desestabilização das fases ordenadas.

Na figura 6.6, apresentamos o diagrama do estado fundamental (groundstate phase diagram) [107] correspondente aos dados da Tabela 6.2, em unidades de $K J / m o l$. Com o auxilio deste diagrama podemos deduzir as fases estáveis do sistema no limite de baixa temperatura, zero graus Kelvin (0K). Nesta figura, o composto B2 (FeAl) apresenta o menor valor da energia de formação

\begin{tabular}{rcl}
\hline Estequiometria & Estrutura & p. interação de cluster $\left[k_{B} \mathrm{~K}\right]$ \\
\hline $\mathrm{FeAl}$ & $\mathrm{B} 2$ & $w_{F e, A l}^{(1)}=-1064.3$ \\
$\mathrm{Fe}_{3} \mathrm{Al}$ & $\mathrm{D0}_{3}$ & $w_{F e, A l}^{(2)}=+757.91$ \\
$\mathrm{FeAl}$ & $\mathrm{B} 32$ & $\tilde{w}_{F e, A l, F e, A l}=-502.2$ \\
$\mathrm{FeAl}_{3}$ & $\mathrm{D0}_{3}$ & $\tilde{w}_{F e, A l, A l, A l}=-261.64$ \\
\hline
\end{tabular}

Tabela 6.3: Parâmetros de interação do cluster em unidades de constante de Boltzmann $\left(1 k_{B} \mathrm{k}=8.3145 \mathrm{~J} \mathrm{~mol}^{-1}\right)$. 


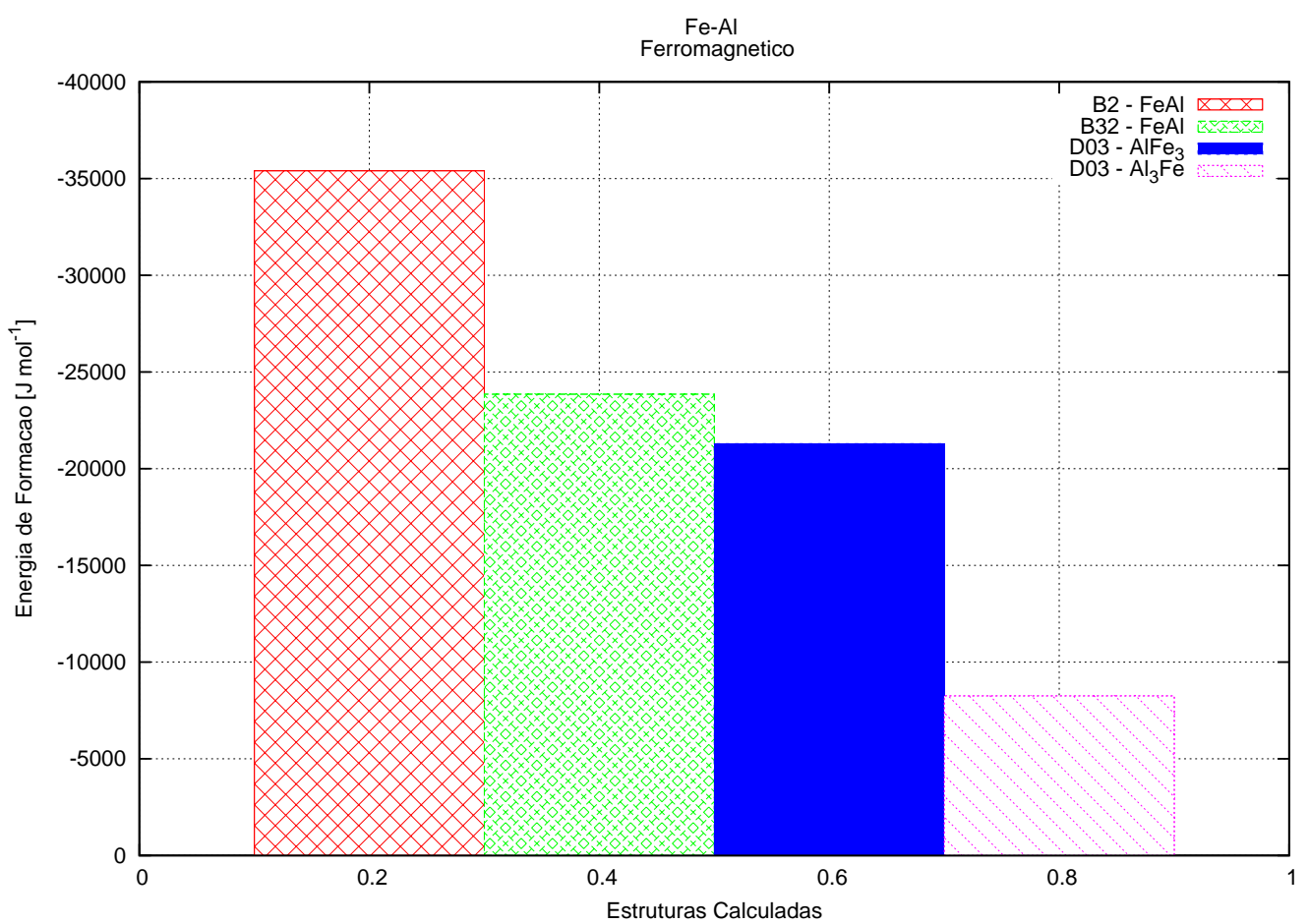

Figura 6.5: Energia de formação dos compostos usados para construir a base para a expansão de cluster do sistema Fe-Al ferromagnético. 


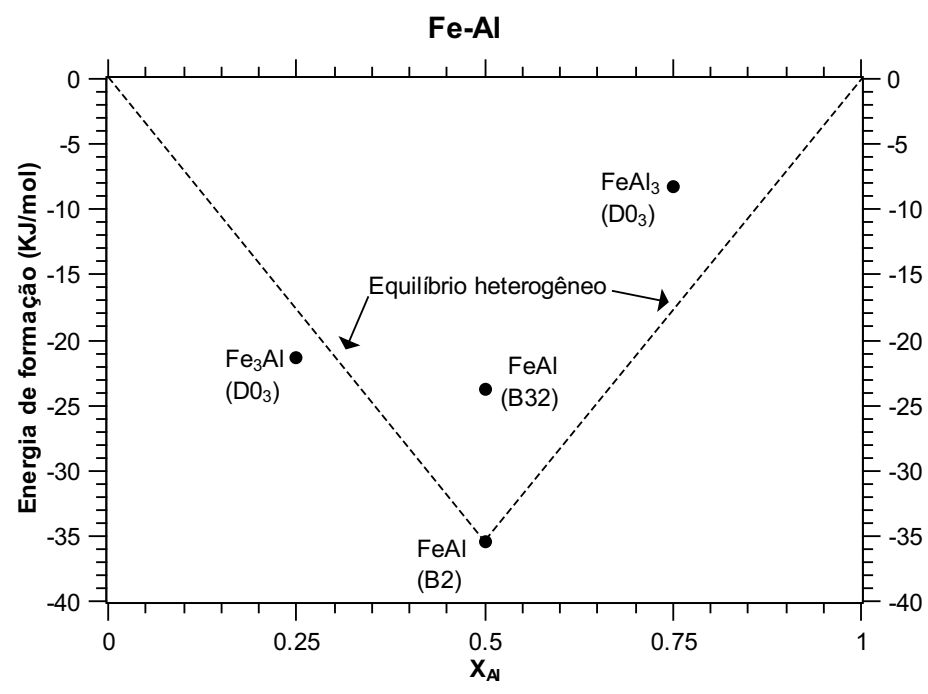

Figura 6.6: Diagrama do estado fundamental do sistema FerroAlumínio (FeAl), cálculo ferromagnético.

e por isto é chamado de composto mais estável, como podemos ver com mais clareza na figura 6.5, na qual é mostrada a hierarquia de estabilidade. As linhas que unem o composto B2 às duas fases $\mathrm{A} 2$ ( $\mathrm{Fe}$ e $\mathrm{Al}$ ) correspondem aos equilíbrios heterogêneos $\mathrm{B} 2+\mathrm{A} 2(\mathrm{Fe})$ do lado esquerdo, e B2+A2 (Al) do lado direito. A comparação da energia de formação das duas fases $D 0_{3}$ $\left(\mathrm{Fe}_{3} \mathrm{Al}\right.$ e $\left.\mathrm{FeAl}_{3}\right)$ com as retas mostra que a energia de formação da fase $\mathrm{DO}_{3} \quad\left(\mathrm{Fe}_{3} \mathrm{Al}\right)$ é mais negativa que a energia do equilíbrio heterogêneo enquanto que a fase $\mathrm{DO}_{3}\left(\mathrm{FeAl} l_{3}\right)$ o equilíbrio heterogêneo tem energia mais negativa, correspondendo este portanto ao equilíbrio estável a $0 \mathrm{~K}$. O sistema apresentará, portanto quatro fases estáveis a baixa temperatura: A2 $(\mathrm{Fe}), \mathrm{A} 2(\mathrm{Al}), \mathrm{B} 2(\mathrm{FeAl})$ e $D 0_{3}\left(F e_{3} A l\right)$, sendo que a região rica em $\mathrm{Al}$ do diagrama de fases $\left(x_{A l}>0.5\right)$ apresentará um equilíbrio heterogêneo entre as fases $\mathrm{A} 2$ do $\mathrm{Al}$ e $\mathrm{B} 2$ do FeAl. É importante ressaltar que estas conclusões se referem apenas ao equilíbrio entre as fases obtidas da estrutura BCC. Na realidade, compostos baseados em outras estruturas [108] são estáveis no sistema Fe-Al para $x_{A l}>0.5$. Os equilíbrios apresentados neste diagrama para $x_{A l}>0.5$ devem ser, portanto, considerados metaestáveis.

$\mathrm{Na}$ figura 6.8, apresentamos o diagrama de fase obtido utilizando os parâmetros da Tabela 6.3. Na figura 6.8, apresentamos o diagrama de fase obtido utilizando os parâmetros da Tabela 6.3. A topologia do diagrama de fase na região rica em $\mathrm{Fe}\left(x_{A l}<0.5\right)$ está de acordo com o diagrama de fase experimental, ilustrado na Figura 6.7. O diagrama aqui obtido pode ser com- 


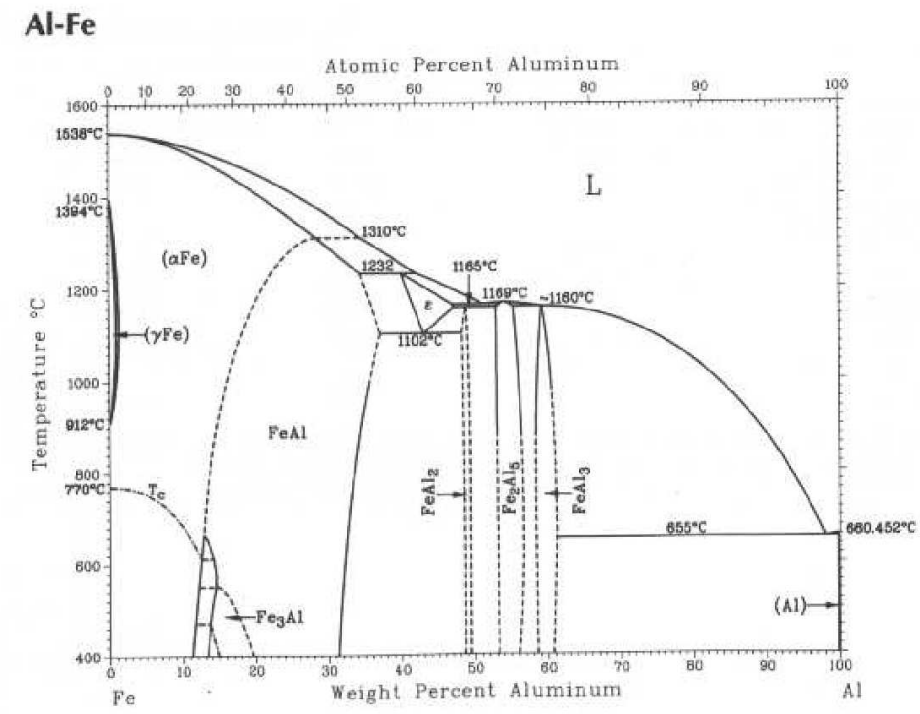

Figura 6.7: Diagrama de fases do sistema Ferro-Alumínio obtido do ASM Metals Handbook-Volume 3-Alloy Phase Diagrams.

parado com os diagramas da literatura [109] onde os cálculos foram realizados sem a inclusão de efeitos relativísticos. Como se poderia esperar para o caso de elementos não pesados, a inclusão de efeitos relativísticos altera muito pouco o diagrama de fases teórico. Conforme realizado na referência [1], realizaremos a seguir por motivo de clareza uma análise didática do diagrama de fase de forma a permitir a sua leitura por não especialistas no assunto. Entre $0.5 \leq x_{A l} \leq 1.0$ temos um campo de duas fases $\mathrm{A} 2(\mathrm{Al})+\mathrm{B} 2$ iniciados a $0 \mathrm{~K}$ e que fecha em um ponto tricrítico está na temperatura $\mathrm{T}=2470 \mathrm{~K}$ e $x_{A l}=0.65$, o máximo da linha de segunda ordem B2/A2 está em T=3225 K e $x_{A l}=0.47$. Comparando com o resultado do cálculo fenomenológico ( $\mathrm{T}=1780$ $\mathrm{K}$ e $\left.x_{A l}=0.50\right)$ vemos que nosso cálculo teórico é $81 \%$ maior, na temperatura. $\mathrm{O}$ outro ponto tricrítico está em $\mathrm{T}=537 \mathrm{~K}$ e $x_{A l}=0.21$ e o máximo da linha de segunda ordem $\mathrm{D}_{3} / \mathrm{B} 2$ está em $\mathrm{T}=587 \mathrm{~K}$ e $x_{A l}=0.27$. Novamente, comparando com o resultado fenomenológico ( $\mathrm{T}=820 \mathrm{~K}$ e $x_{A l}=0.28$ ) para o nosso cálculo teórico é deO estado quasicristalinos da matéria tem sido estudada intensamente no ano passado. Este estado é o personalizado por ordem de longo alcance icosaédrico e foi primeiramente relatada por Shechtman etal.1 e desde então tem sido o tema de muitos experimental estudos $28 \%$ menor na temperatura. Outro dado importante é a comparação entre os valores experimentais da entalpia de formação $\left(\delta^{f} H\right)$ para as fases B2 e $\mathrm{D}_{3}$ a $x_{A l}=0.5$ e $x_{A l}-0.25$, respectivamente $[111,112]$. Estes valores experimentais, após a conversão ao estado de referência usado no presente trabalho 


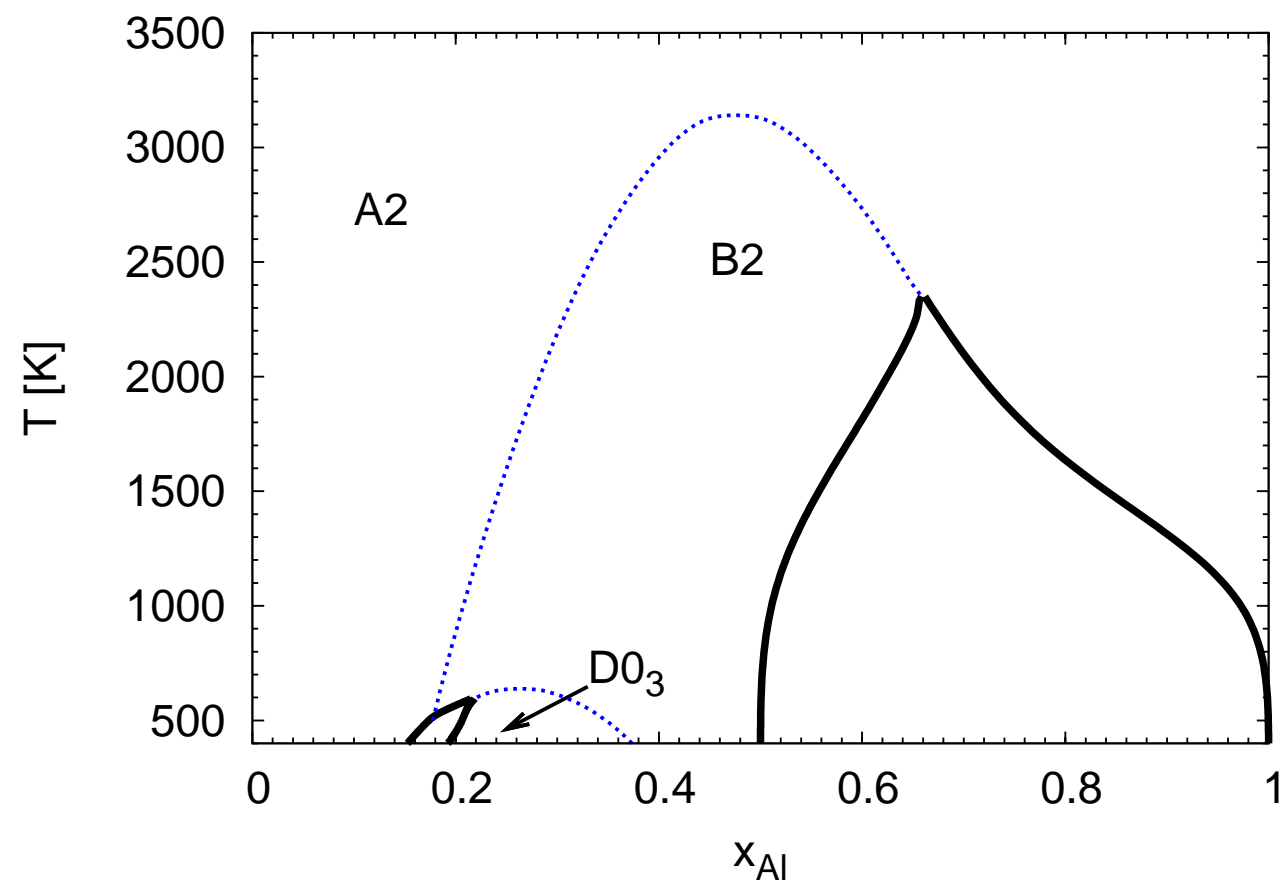

Figura 6.8: Diagrama de fases do sistema Fe-Al com polarização de spin e na aproxi mação escalar relativística obtido neste trabalho. 
fornecem: $-21,3 k \mathrm{~J} / \mathrm{mol}$ para a fase $\mathrm{D0}_{3}\left(\mathrm{Fe}_{3} \mathrm{Al}\right)$ e $-35,4 k \mathrm{~J} / \mathrm{mol}$ para a fase B2 (FeAl). A comparação entre os valores apresentados na tabela 6.2 com os valores experimentais fornecem valores idênticos. Apesar de termos considerado no cálculo de estrutura eletrônica os graus de liberdade magnética eles foram ignorados no cálculo termodinâmico. A inclusão dos graus de liberdade magnéticos no cálculo termodinâmico do CVM foi realizado na referência [109] para cálculos não relativísticos. Uma vez que o diagrama de fases 6.8 com a inclusão de efeitos relativísticos não apresentou nenhuma variação significativa em relação ao diagrama de fases [109] sem a inclusão dos graus de liberdade magnético no CVM, não esperamos que esta inclusão altere o diagrama de fases apresentado na referência [109] obtido com a inclusão dos graus de liberdade magnético no CVM. O momento magnético do Fe obtido através do cálculo ab-initio de estrutura eletrônica para os compostos do sistema Fe-Al é apresentado na Tabela 6.1 


\subsection{Sistemas $\mathrm{Fe}-\mathrm{Cr}$}

O sistema binário Fe-Cr é a base para uma grande classe de importantes materiais da engenharia conhecido como aços inoxidáveis. Aços inoxidáveis combinam boa resistência à corrosão, a boas propriedades mecânicas.É possível ocorrer fragilização devido a uma reação de separação de fases onde a ferrita se decompoem em regiões que são ricas em Ferro ou Cromo. Algumas outras caracterícsticas das ligas de Fe-Cr também são interessantes, tal como formação de vidros de spin,uma lenta formação da fase $\sigma$, e uma forte resistência ao inchamento em um ambiente irradiado. Tudo isso exige uma compreensão da termodinâmica do sistema Fe-Cr. Nesta seção obteremos o diagrama de fases de equilibrio e algumas propriedades termodinâmicas do sistema Fe-Cr. Faremos uma reavaliação da descrição termodinâmica do binário $\mathrm{Fe}-\mathrm{Cr}$, de tal modo a vermos o que ocorre no limite de solubilidade na região rica em ferro. Para vermos se nosso diagrama Ferro-Cromo concorda com o modelo cohesivo ou o calphad, ou seja se o limite de solubilidade na região rica em ferro é igual ou diferente do gap padrão de miscibilidade.

Para podermos construir o diagrama de fases do sistema binário $\mathrm{Fe}-\mathrm{Cr}$ utilizamos o funcional de troca e correlação GGA-PBE [48], bem como o métodos FP-LAPW. Todos os cálculos de estrutura eletrônica realizados foram escalar relativísticos, ou seja, procedimento análogo ao realizado para os sistema anteriores. Desta forma encontramos quais eram as estruturas otimizadas $6.9,6.10,6.11$ e 6.12 . A partir do momento em que já tinhamos as estruturas otimizadas fomos realizar o cálculo em si das energias no estado fundamental. Calculamos a $E_{T}$ dos compostos no estado fundamental, $\mathrm{FeCr}$ (nas estruturas $\mathrm{B} 2$ e $\mathrm{B} 32, \mathrm{Fe}_{3} \mathrm{Cr}$ e $\mathrm{FeCr}_{3}$ (na estrutura $\mathrm{D0}_{3}$ ) todos eles com a estrutura BCC, de forma análoga ao sistema Fe-Al. Para todas as estruturas foi utilizado o raio de muffin-tin da esfera no sítio atômico de $R m t=2.00$ u.a..

Os valores encontrados para as energias no estado fundamental são apresentados na Tabela (6.4), juntamente com os valores encontrados para o parâmetro de rede de equilíbrio e o momento magnético médio no átomo de Ferro. Com as energias totais no estado fundamental, calculamos as energias de formação para o sistema Fe-Cr. Todas as energias deram valores positivos, portanto nenhuma das estruturas da liga de Fe-Cr é estável, semelhante ao caso Fe-Mo. As fases mais estáveis no estado fundamental, são as A2 (Fe e $\mathrm{Cr}$ ) sendo que o diagrama de fases será dominado por um equilíbrio heterogêneo possuindo um domo de imiscibilidade bem característico. $\mathrm{Na}$ figura 6.13, podemos ver a hierarquia das energias de formação para cada estruturas. É possível observar que não se manteve a ordem observada no caso Fe-Al ou no Fe-Mo. Para o sistema Fe-Cr houve uma mudança entre 


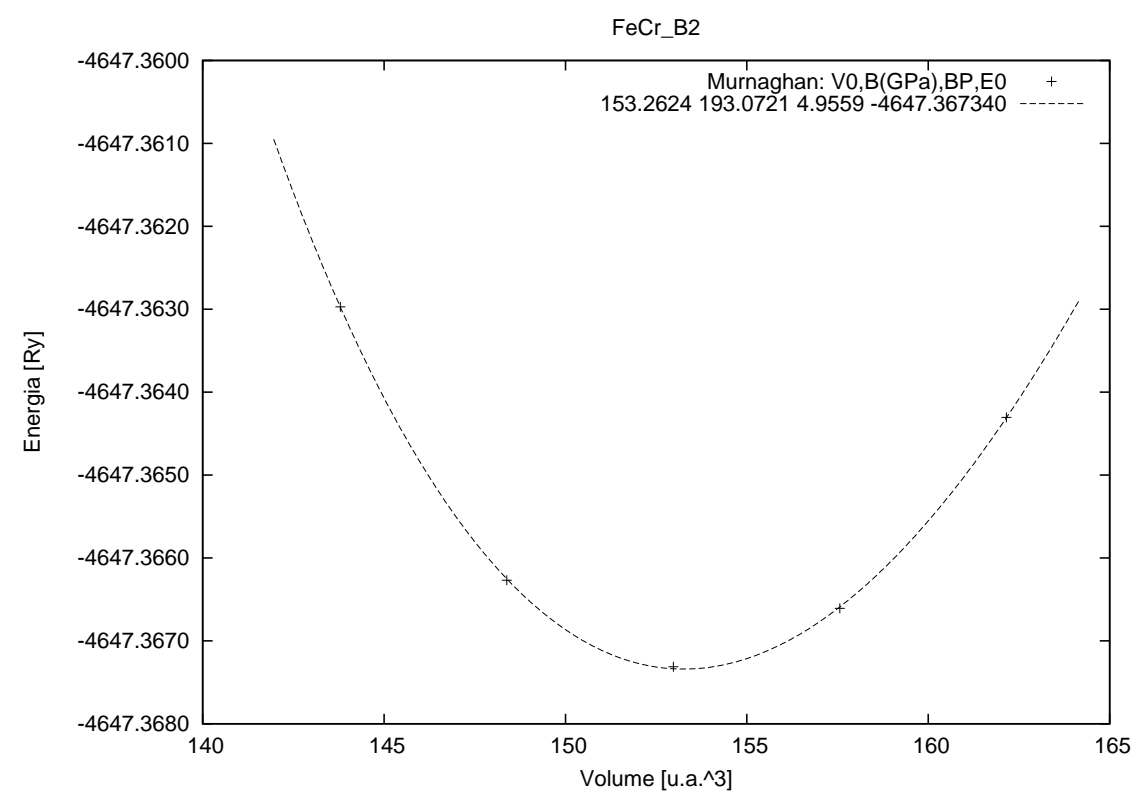

Figura 6.9: Otimização estrutural da liga binária Ferro-Cromo (FeCr-B2) para o caso FP-LAPW. Na legenda temos: $V_{0}$ é o volume mínimo em unidades atômicas; B(GPa) é o Bulk módulo, BP é o Derivada do Bulk Módulo e $E_{0}$ a energia mínima.

Tabela 6.4: Energia total calculada para os seis compostos formando a base para a expansão de cluster aplicada ao sistema Fe-Cr com todos os parâmetros de controle otimizados. Para todos os calculos $R k \max =\mathrm{Lmax}=10$, número de pontos K na zona irredutível de Brillouin foi de 286. O momento magnético médio no átomo de ferro (MM) é apresentado (em magneton de Bohr, $\mu_{B}$ ). Os parâmetros de rede são expressados em nm e os valores da das energias são representados em Rydberg (Ry) por formula unitária (1 Ry $=2.1798741 \times$ $\left.10^{-18} \mathrm{~J}\right)$.

\begin{tabular}{clcccc}
\hline Calculo & Estequiometria & Estrutura & $a_{0}$ & $\mathrm{MM}$ & $E_{T}$ \\
\hline $\mathrm{FM}$ & $\mathrm{Fe}$ & $\mathrm{A} 2$ & 0.283 & +2.24 & -2545.612324 \\
$\mathrm{AFM}$ & $\mathrm{Cr}$ & $\mathrm{A} 2$ & 0.285 & 0 & -2101.784485 \\
$\mathrm{FM}$ & $\mathrm{FeCr}$ & $\mathrm{B} 2$ & 0.283 & +1.42 & -4647.368794 \\
$\mathrm{FM}$ & $\mathrm{FeCr}$ & $\mathrm{B} 32$ & 0.566 & +1.86 & -9294.769067 \\
$\mathrm{FM}$ & $\mathrm{Fe}_{3} \mathrm{Cr}$ & $\mathrm{D0}_{3}$ & 0.567 & +2.13 & -9738.604355 \\
$\mathrm{FM}$ & $\mathrm{FeCr}_{3}$ & $\mathrm{D}_{3}$ & 0.568 & +1.71 & -8850.930037 \\
\hline
\end{tabular}




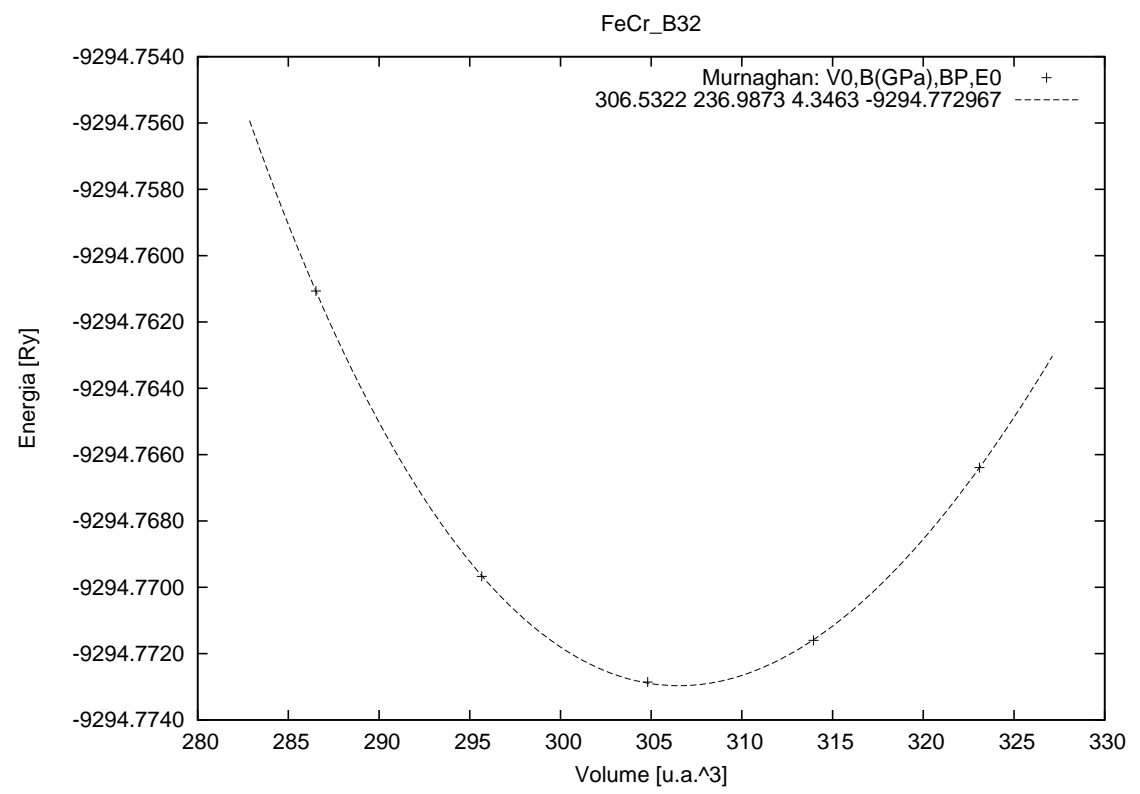

Figura 6.10: Otimização estrutural da liga binária Ferro-Cromo (FeCr-B32) para o caso FP-LAPW. Na legenda temos: $V_{0}$ é o volume mínimo em unidades atômicas; $\mathrm{B}(\mathrm{GPa})$ é o Bulk módulo, BP é o Derivada do Bulk Módulo e $E_{0}$ a energia mínima.

\begin{tabular}{ccccc}
\hline Sistema & Fase & Ry & $\mathrm{kJ} / \mathrm{mol}$ & $K_{B} K$ \\
\hline \hline FeCr & B2 & +0.0140 & +18.389 & +2211.722 \\
FeCr & B32 & +0.0061 & +8.058 & +969.143 \\
Fe3Cr & D03 & +0.0043 & +5.613 & +675.125 \\
FeCr3 & D03 & +0.0089 & +11.731 & +1410.871 \\
\hline
\end{tabular}

Tabela 6.5: Energia de formação do sistema Fe-Cr com polarização de spin e sem acoplamento spin orbita, utilizando o método FP-LAPW. 


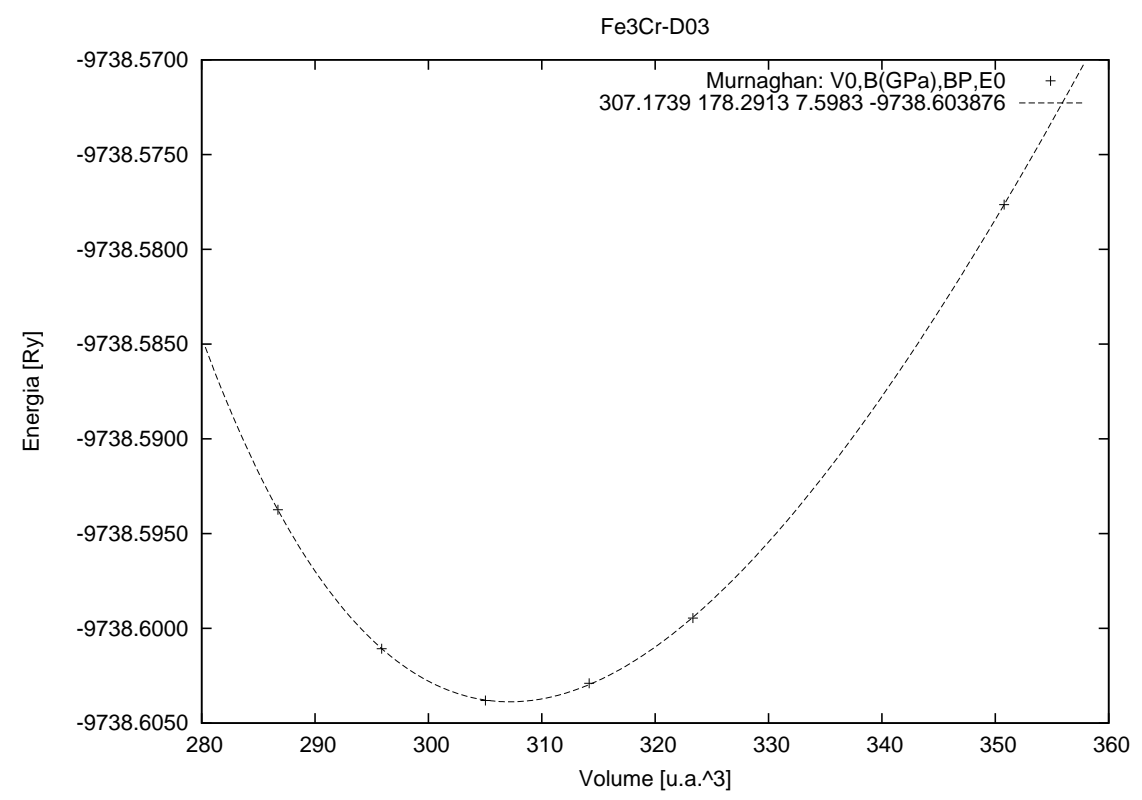

Figura 6.11: Otimização estrutural da liga binária Ferro-Cromo (Fe3Cr-D03) para o caso FP-LAPW. Na legenda temos: $V_{0}$ é o volume mínimo em unidades atômicas; $\mathrm{B}(\mathrm{GPa})$ é o Bulk módulo, BP é o Derivada do Bulk Módulo e $E_{0}$ a energia mínima.

as estruturas B32 e $\mathrm{D}_{3}\left(\mathrm{FeCr}_{3}\right)$ observando os valores absolutos. Na Figura (6.14) apresentamos o diagrama de fases do estado fundamental. Pela análise da figura concluímos que a linha correspondente ao equilíbrio heterôgeneo A2 - Fe e A2 - Cr será mais negativa que a energia de formação de todos os outros compostos do sistema. Desta forma o diagrama de fases será dominado por este equilíbrio heterogêneo e apresentará um domo de miscibilidade como previamos.

Com os valores da energia total no estado fundamental calculamos os parâmetros de interação do sistema $\mathrm{Fe}-\mathrm{Cr}$ para podermos contruir a termodinâmica do sistema e por fim obtermos o diagrama de fases. Os valores obtidos são mostrados na Tabela (6.6).

Na Figura (6.16) mostramos o diagrama obtido por termodinâmica computacional utilizando a metodologia do formalismo CALPHAD. Neste sistema, a comparação direta não é viável pois há varias fases (como $\sigma$, liquida,...) que nossa metodologia não consegue calcular. Contudo a fase BCC é possível comparar qualitativamente. Na Figura (6.17) apresentamos o Diagrama de Fases do sistema Fe-Cr. Conforme o que foi discutido no diagrama de fases do estado fundamental, o diagrama de fases é dominado por um campo de duas fases, A2 - (Fe) + A2 - ( Cr), chamado de domo de imiscibili- 


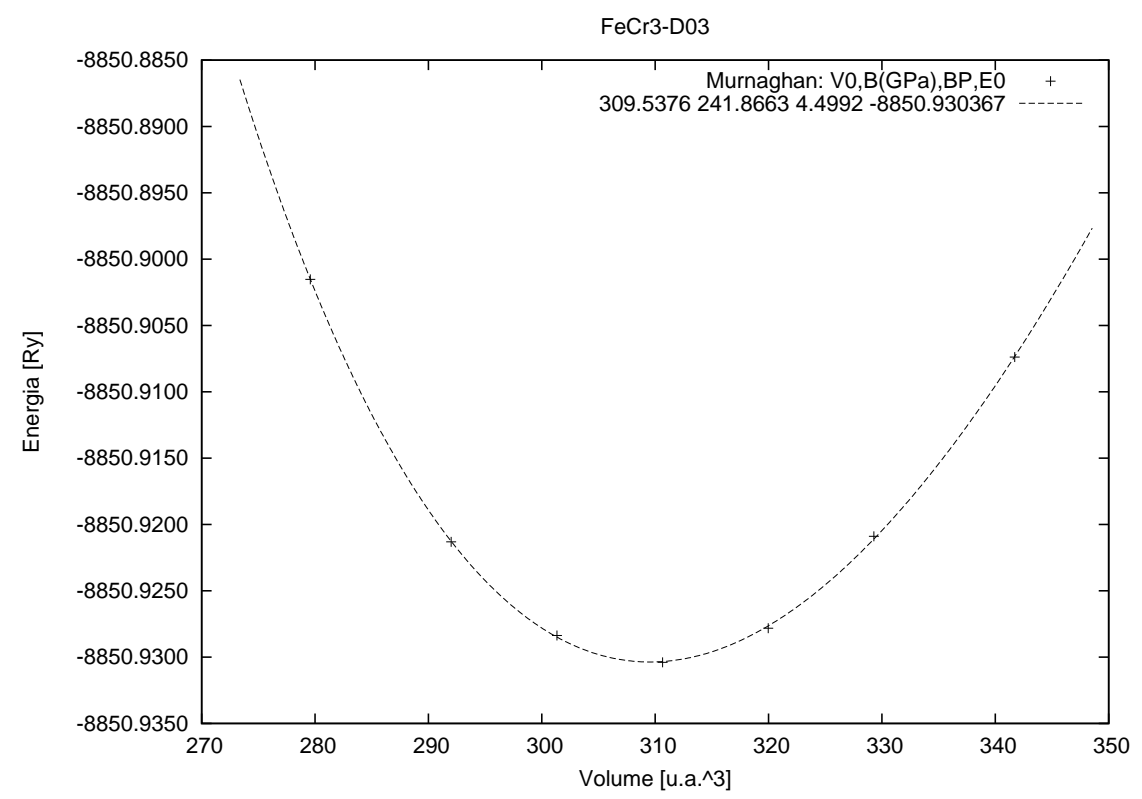

Figura 6.12: Otimização estrutural da liga binária Ferro-Cromo (FeCr3-D03) para o caso FP-LAPW. Na legenda temos: $V_{0}$ é o volume mínimo em unidades atômicas; $\mathrm{B}(\mathrm{GPa})$ é o Bulk módulo, BP é o Derivada do Bulk Módulo e $E_{0}$ a energia mínima.

\begin{tabular}{rcl}
\hline Estequiometria & Estrutura & p. interação de cluster $\left[k_{B} \mathrm{~K}\right]$ \\
\hline $\mathrm{FeCr}$ & $\mathrm{B} 2$ & $w_{F e, C r}^{(1)}=+552.93$ \\
$\mathrm{Fe}{ }_{3} \mathrm{Cr}$ & $\mathrm{D}_{3}$ & $w_{F e, C r}^{(2)}=+203.34$ \\
$\mathrm{FeCr}$ & $\mathrm{B} 32$ & $\tilde{w}_{F e, C r, F e, C r}=-124.46$ \\
$\mathrm{FeCr}_{3}$ & $\mathrm{D}_{3}$ & $\tilde{w}_{F e, C r, C r, C r}=-122.62$ \\
\hline
\end{tabular}

Tabela 6.6: Parâmetros de interação do cluster em unidades de constante de Boltzmann $\left(1 k_{B} \mathrm{k}=8.3145 \mathrm{~J} \mathrm{~mol}^{-1}\right)$. 


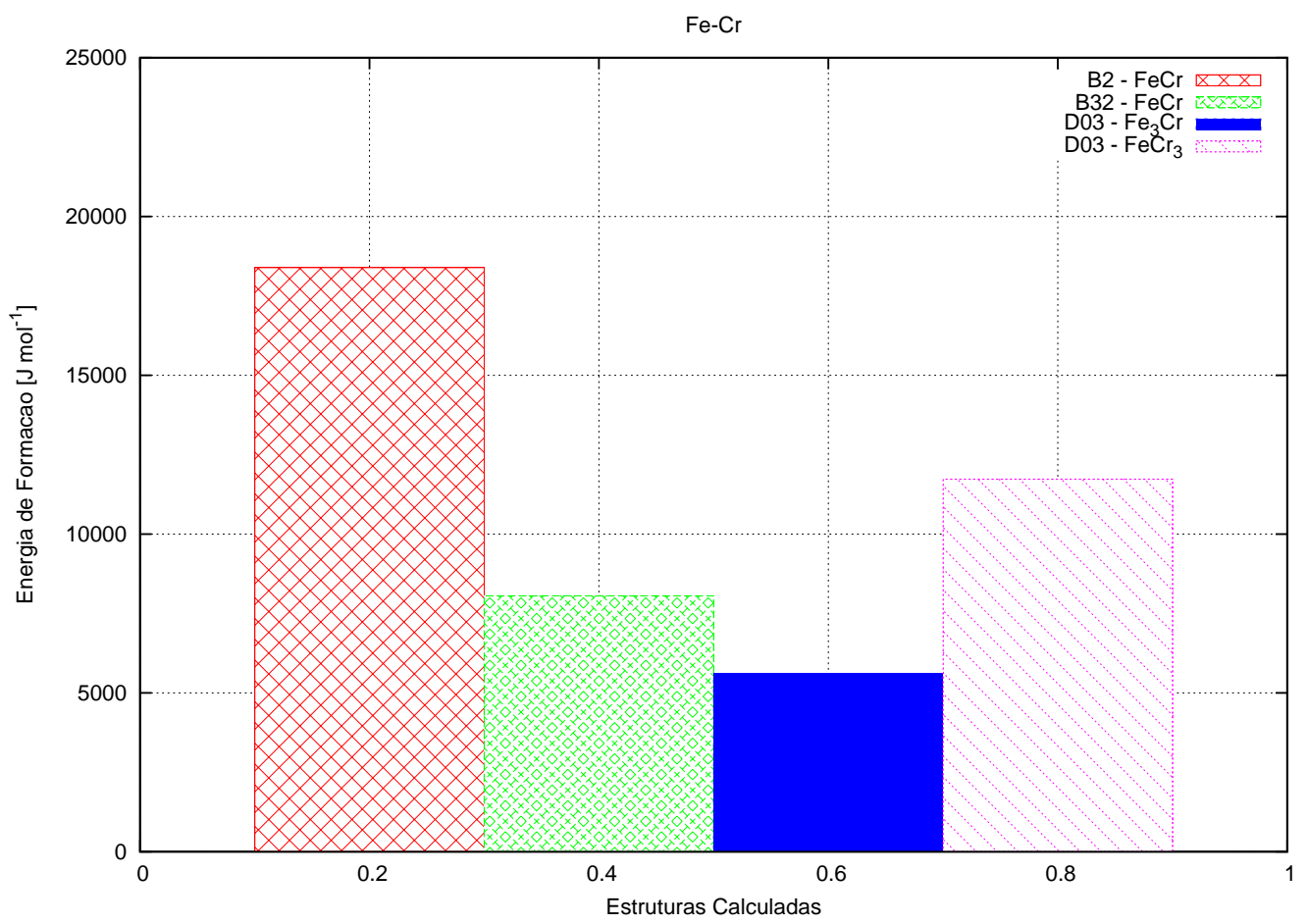

Figura 6.13: Energia de formação dos compostos usados para construir a base para a expansão de cluster do sistema Fe-Cr. 


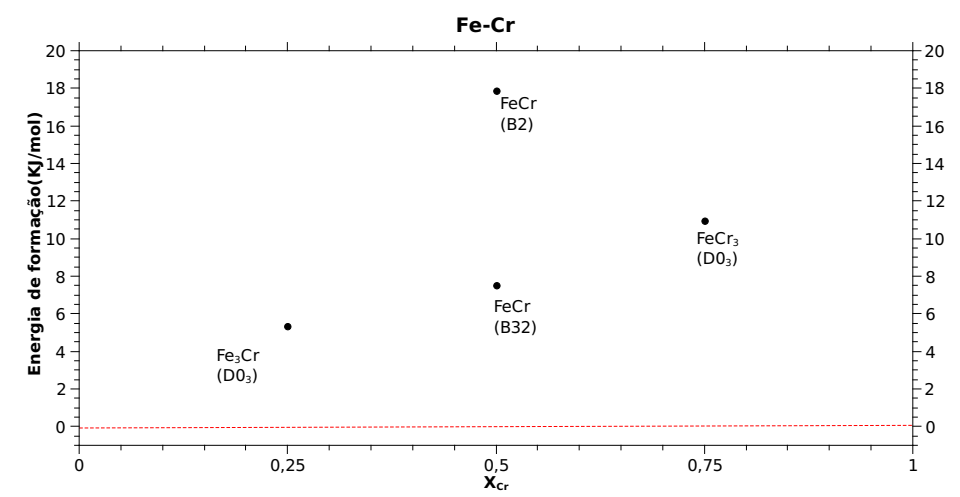

Figura 6.14: Diagrama do estado fundamental do sistema FerroCromo (FeCr), cálculo ferromagnético.

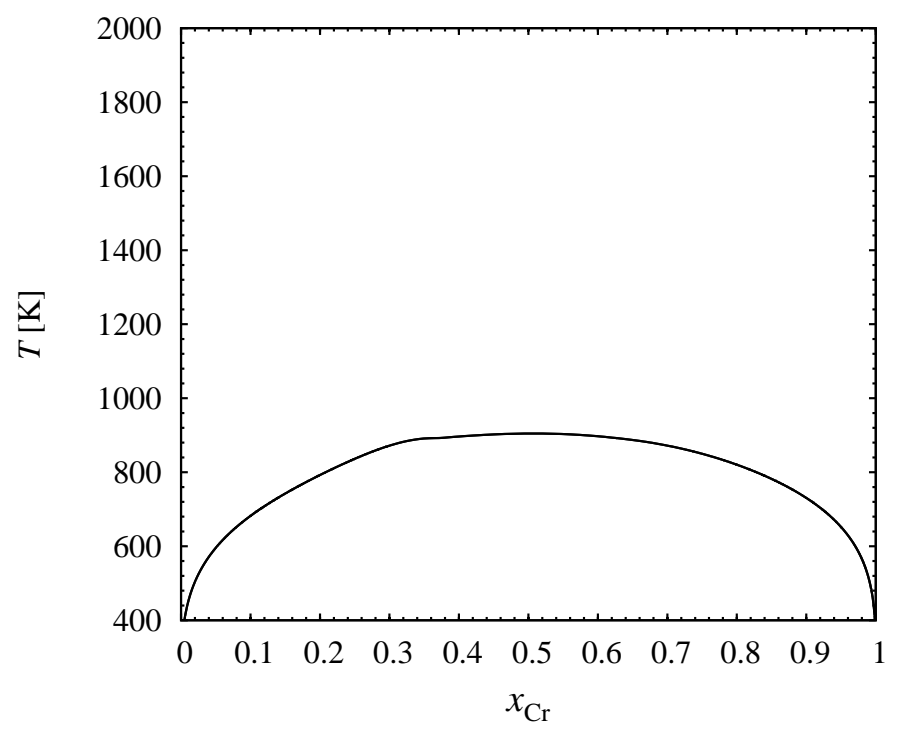

Figura 6.15: Diagrama de fases do sistema Ferro-Cromo obtido via metodologia CALPHAD, domo de imiscibilidade.

dade. Acima da linha binodal só temos a fase desordenada A2. Comparando os dois diagramas de fases (6.16) e (6.17) podemos ver qualitativamente que eles possuem comportamentos próximos. O diagrama ab-initio esta com a temperatura super estimada, mas esse dado já era esperado pela metodologia. O comportamento na região rica em Fe mostra que não há solubilidade de cromo no estado fundamental. Nosso resultado não considera a presença de ordenamento magnético de $\mathrm{Cr}$ na liga com $<10 \%$ de $\mathrm{Cr}$, pois isto esta 


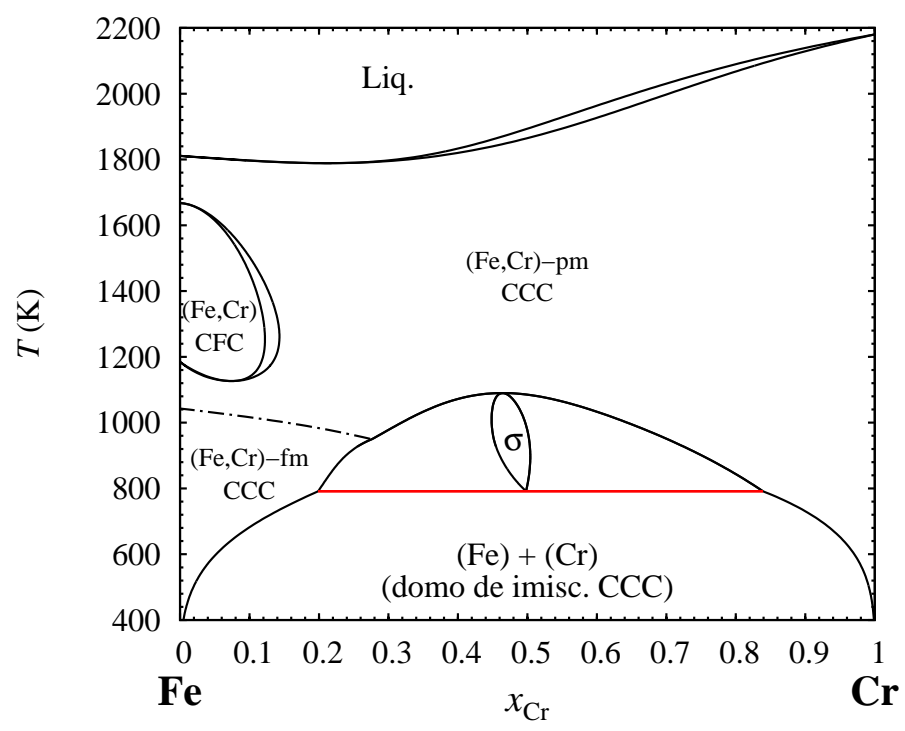

Figura 6.16: Diagrama de fases do sistema Ferro-Cromo obtido atraves da metodologia CALPHAD.

fora do escopo do trabalho. Concordando com os resultados CALPHAD e discordando do resultado do modelo proposto na referência(colocar Trabalho belga-cohercivo).

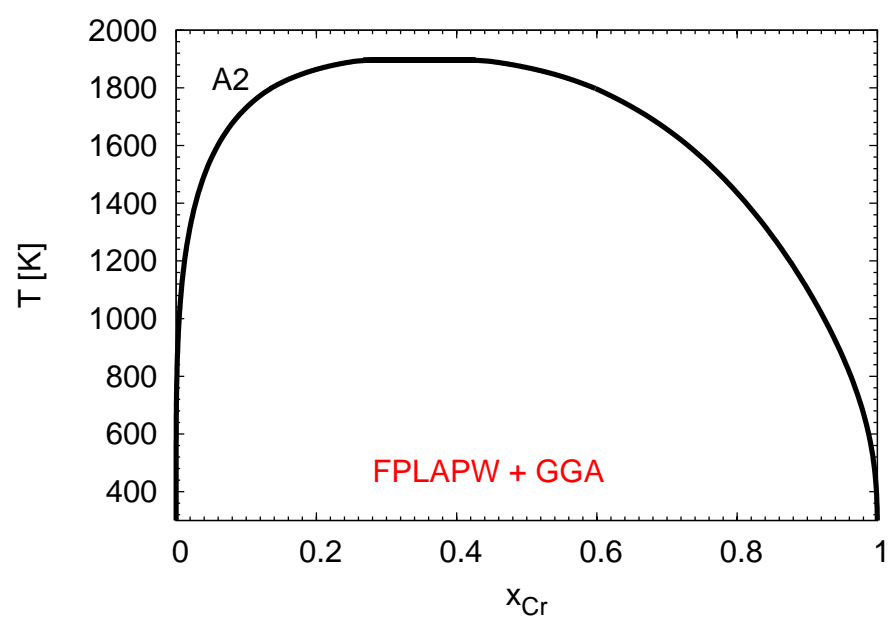

Figura 6.17: Diagrama de fases do sistema Ferro-Cromo obtido neste trabalho. 


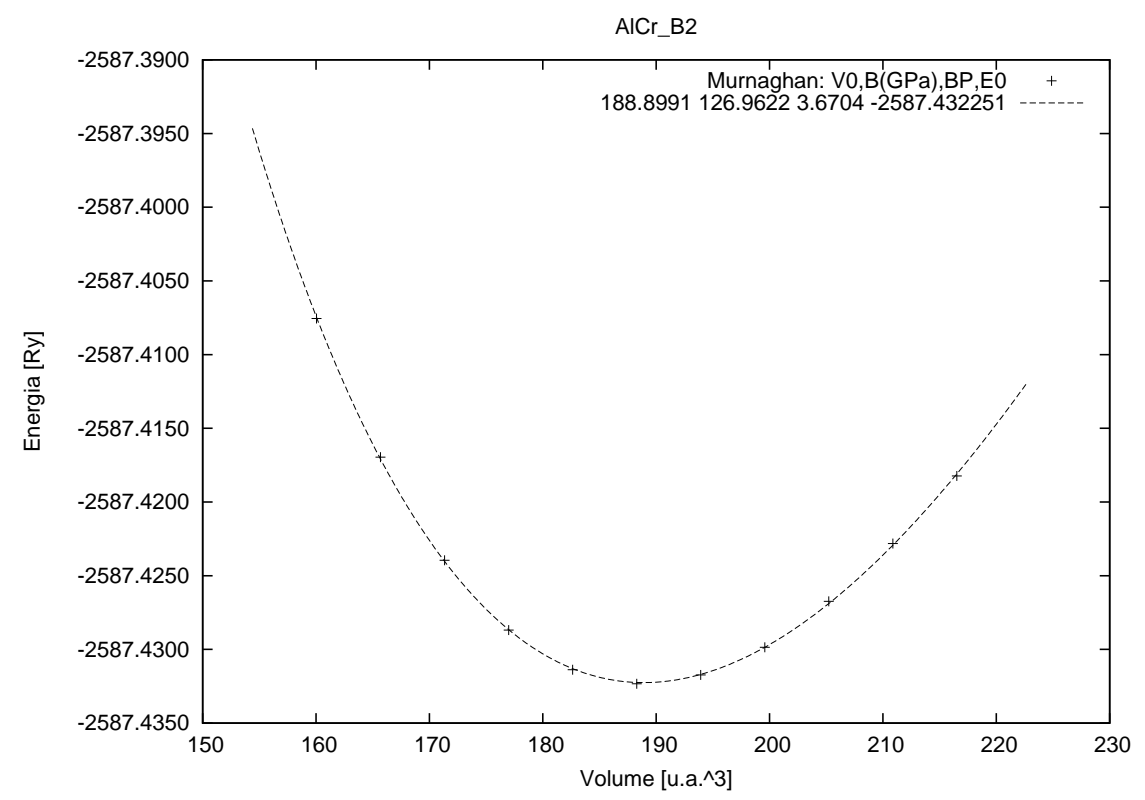

Figura 6.18: Otimização estrutural da liga binária Alumínio-Cromo (AlCrB2) para o caso FP-LAPW. Na legenda temos: $V_{0}$ é o volume mínimo em unidades atômicas; $\mathrm{B}(\mathrm{GPa})$ é o Bulk módulo, BP é o Derivada do Bulk Módulos e $E_{0}$ a energia mínima.

\subsection{Sistemas Al-Cr}

O estado quasicristalino da matéria tem sido estudado intensamente nos últimos anos. Este estado é caracterizado por ordem de longo alcance icosaédrica e foi primeiramente relatado por Shechtman e desde então tem sido o tema de muitos estudos experimentais. Realizamos os cálculos com o método FP-LAPW considerando os efeitos relativísticos. O comportamento do Cr é bem intrincado, apresentando ondas de spin. Nas Figuras (6.18), (6.19), (6.20) e (6.21) mostramos o resultado final do processo de otimização estrutural. 


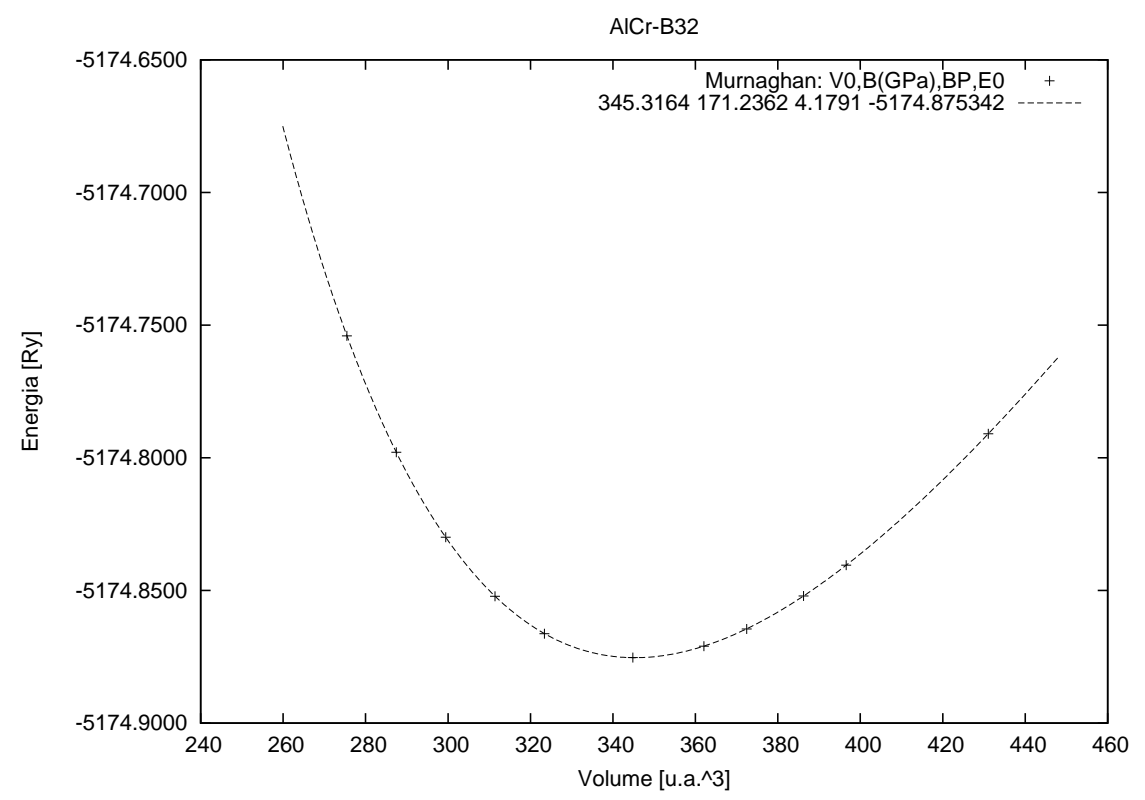

Figura 6.19: Otimização estrutural da liga binária Alumínio-Cromo (AlCrB32) para o caso FP-LAPW. Na legenda temos: $V_{0}$ é o volume mínimo em unidades atômicas; $\mathrm{B}(\mathrm{GPa})$ é o Bulk módulo, BP é o Derivada do Bulk Módulo e $E_{0}$ a energia mínima.

Com as estruturas otimizadas, calculamos a energia total para o estado fundamental. O procedimento para atingir a convergência no estado fundamental é análogo aos casos anteriores (Fe-Mo, Fe-Al, Fe-Cr). Os resultados tanto para a energia total no estado fundamental, quanto para o parâmetro de rede de equilíbrio e o momento magnético médio no átomo de Cromo podem ser vistos na Tabela (6.7). Na Tabela(6.8), apresentamos a energia de formação dos quatro compostos do sistema Al-Cr. Podemos ver que o composto B32 (Al-Cr) possui a mais baixa energia de formação. No estado fundamental, esta estrutura é a mais estável nesta estequiometria. Na Figura (6.22) podemos ver que os únicos compostos estáveis do sistema será A2-Cr, A2-Al e B32. Na Tabela (6.9) mostramos os parametros de interação calculados com os dados da Tabela (6.8) Na Figura (6.23) vemos o diagrama de fases no estado fundamental. Podemos ver que a única fase ordenada que será estável no diagrama de fases será a fase B32 (Al-Cr), pois os outros compostos são menos estáveis que a linha do equílibrio heterogêneo (B32-A2).

Na Figura (6.25) apresentamos o diagrama de fases do sistema Al-Cr. Podemos ver a presença isolada da fase B32, conforme foi mostrado na análise do estado fundamental. A fase B32 é estável a partir de $x_{A l} \approx 0.41$ até $x_{A l} \approx 0.46$ no diagrama de fases. O equilíbrio entre as fases B32 e A2 é de primeira or- 


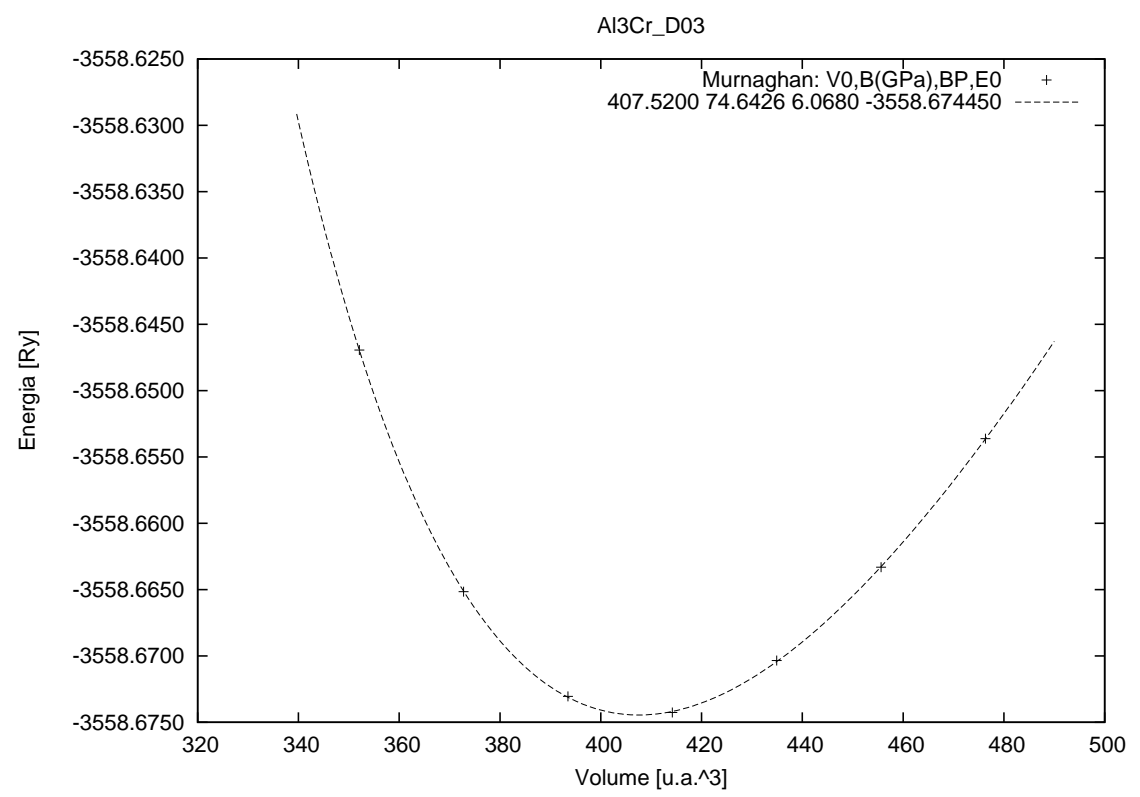

Figura 6.20: Otimização estrutural da liga binária Alumínio-Cromo (Al3CrD03) para o caso FP-LAPW. Na legenda temos: $V_{0}$ é o volume mínimo em unidades atômicas; $\mathrm{B}(\mathrm{GPa})$ é o Bulk módulo, BP é o Derivada do Bulk Módulo e $E_{0}$ a energia mínima.

Tabela 6.7: Energia total calculada para os seis compostos formando a base para a expansão de cluster aplicada ao sistema Al-Cr. Para todos os calculos Rkmax $=$ Lmax $=10$, número de pontos $\mathrm{K}$ na zona irredutível de Brillouin foi de 286. O momento magnético médio no átomo de cromo (MM) é apresentado (em magneton de Bohr, $\mu_{B}$ ). Os parâmetros de rede são expressados em nm e os valores da das energias são representados em Rydberg (Ry) por formula unitária $\left(1 \mathrm{Ry}=2.1798741 \times 10^{-18} \mathrm{~J}\right)$.

\begin{tabular}{clcccc}
\hline Calculo & Estequiometria & Estrutura & $a_{0}$ & $\mathrm{MM}$ & $E_{T}$ \\
\hline $\mathrm{FM}$ & $\mathrm{AlCr}$ & $\mathrm{B} 2$ & 0.304 & +2.29 & -2587.432519 \\
$\mathrm{FM}$ & $\mathrm{AlCr}$ & $\mathrm{B} 32$ & 0.589 & $2.10^{-5}$ & -5174.877909 \\
$\mathrm{FM}$ & $\mathrm{CrAl}_{3}$ & $\mathrm{D0}_{3}$ & 0.623 & +2.47 & -3558.675232 \\
$\mathrm{FM}$ & $\mathrm{Cr}_{3} \mathrm{Al}$ & $\mathrm{D0}_{3}$ & 0.591 & $4.5 .10^{-3}$ & -6791.002128 \\
\hline
\end{tabular}




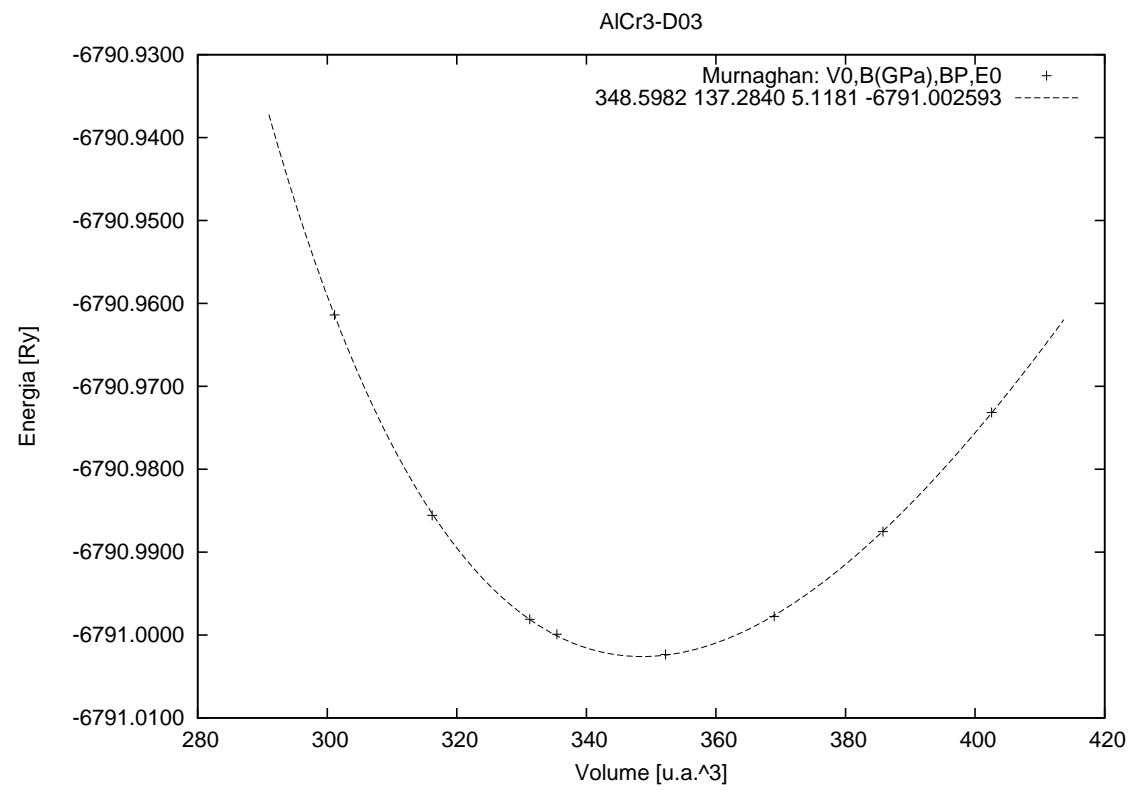

Figura 6.21: Otimização estrutural da liga binária Alumínio-Cromo (AlCr3D03) para o caso FP-LAPW. Na legenda temos: $V_{0}$ é o volume mínimo em unidades atômicas; $\mathrm{B}(\mathrm{GPa})$ é o Bulk módulo, BP é o Derivada do Bulk Módulo e $E_{0}$ a energia mínima.

\begin{tabular}{ccccc}
\hline Sistema & Fase & Ry & $\mathrm{kJ} / \mathrm{mol}$ & $K_{B} K$ \\
\hline \hline $\mathrm{AlCr}$ & $\mathrm{B} 2$ & -0.0054 & -7.126 & -856.994 \\
$\mathrm{AlCr}$ & $\mathrm{B} 32$ & -0.0086 & -11.350 & -1365.079 \\
$\mathrm{Al}_{3} \mathrm{Cr}$ & $\mathrm{D} 03$ & +0.0059 & +6.823 & +820.593 \\
$\mathrm{AlCr}_{3}$ & D03 & -0.0029 & -3.773 & -453.730 \\
\hline
\end{tabular}

Tabela 6.8: Energia de formação do sistema Al-Cr com polarização de spin e sem acoplamento spin orbita, utilizando o método FP-LAPW. 


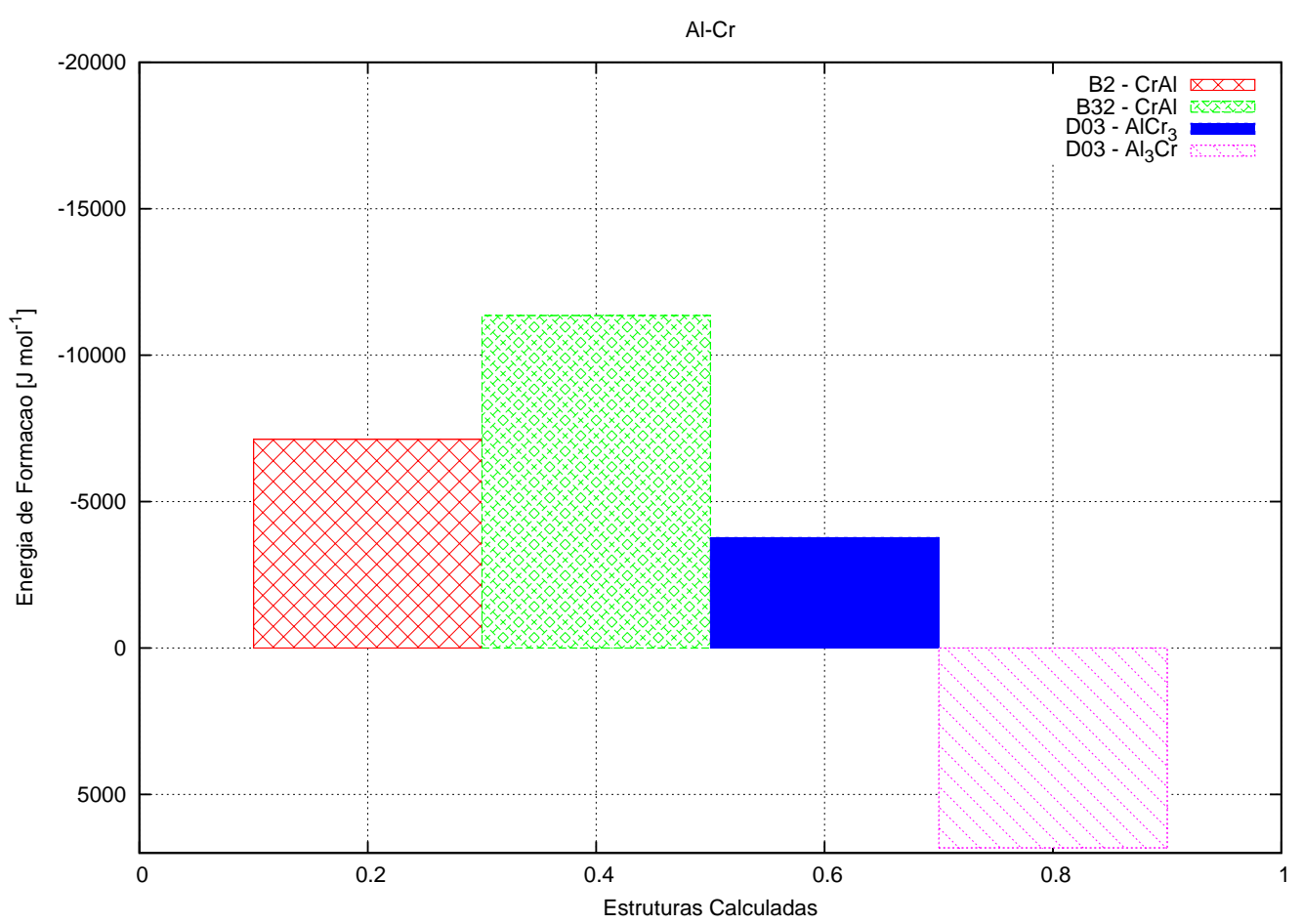

Figura 6.22: Energia de formação dos compostos usados para construir a base para a expansão de cluster do sistema Al-Cr. 


\begin{tabular}{rcl}
\hline Estequiometria & Estrutura & p. interação de cluster $\left[k_{B} \mathrm{~K}\right]$ \\
\hline $\mathrm{AlCr}$ & $\mathrm{B} 2$ & $w_{A l, C r}^{(1)}=-214.25$ \\
$\mathrm{Al}_{3} \mathrm{Cr}$ & $\mathrm{D}_{3}$ & $w_{A l, C r}^{(2)}=-16.82$ \\
$\mathrm{AlCr}$ & $\mathrm{B} 32$ & $\tilde{w}_{A l, C r, A l, C r}=-147.69$ \\
$\mathrm{AlCr}_{3}$ & $\mathrm{D}_{3}$ & $\tilde{w}_{A l, C r, C r, C r}=+212.39$ \\
\hline
\end{tabular}

Tabela 6.9: Parâmetros de interação do cluster em unidades de constante de Boltzmann $\left(1 k_{B} \mathrm{k}=8.3145 \mathrm{~J} \mathrm{~mol}^{-1}\right)$.

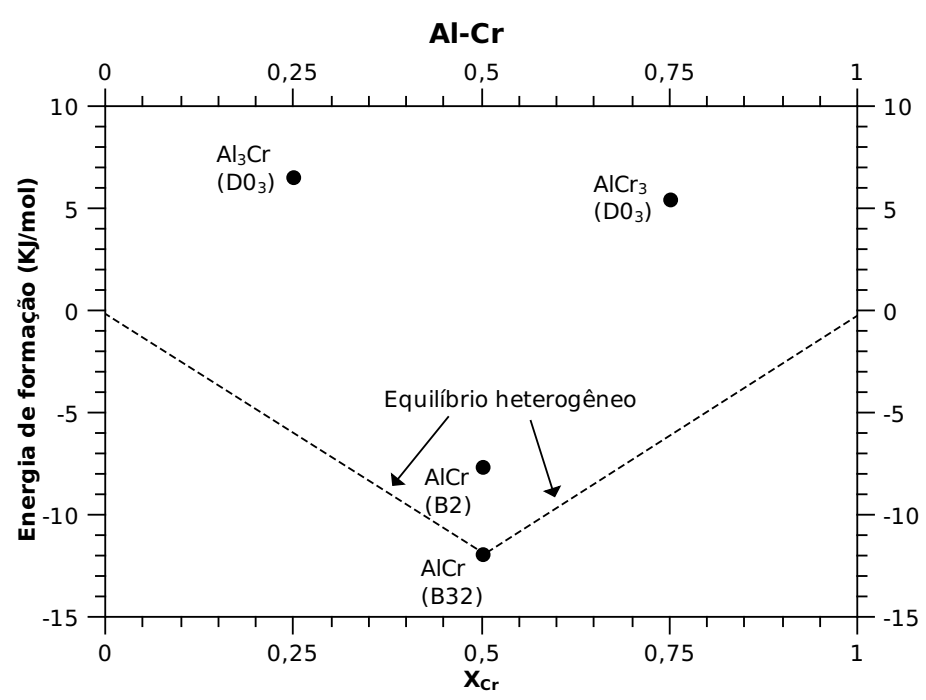

Figura 6.23: Diagrama do estado fundamental do sistema AlumínioCr (AlCr), cálculo ferromagnético.

dem em todas as temperaturas e o campo de fase B32 é, portanto limitado por dois campos de duas fases $\mathrm{A} 2+\mathrm{B} 32$, um para os teores de $\mathrm{Al}$ maiores que $x_{A l} \approx 0.45$ e outro para teores menores $x_{A l} \approx 0.41$.

$\mathrm{Na}$ figura (6.24) apresentamos o diagrama de fases experimental Al-Cr. Entretanto apenas uma fase é baseada na rede CCC. A solução sólida rica em Cr (Cr-A2), estável até $x_{A l} \approx 0.56$ limita-se a uma faixa de temperaturas de 800 a 1400 graus centigrados. Helander e Toluchko [113] identificaram para temperaturas mais baixas a formação da fase $\mathrm{AlCr}_{2}$ que limita a solubilidade de aluminio na fase cúbica de corpo centrado. Na referência [113] Tolochko observou uma reflexão de difração de raio-x em uma liga com $x_{C r}=0.648 \mathrm{e}$ atribuiram esta reflexão a uma fase B2 que seria estável. A evidência experimental é muito sutil. Nosso resultado sugere que se alguma fase ordenada fosse estar nessa região, esta seria B32, e não B2. Liang e autores [114] re- 


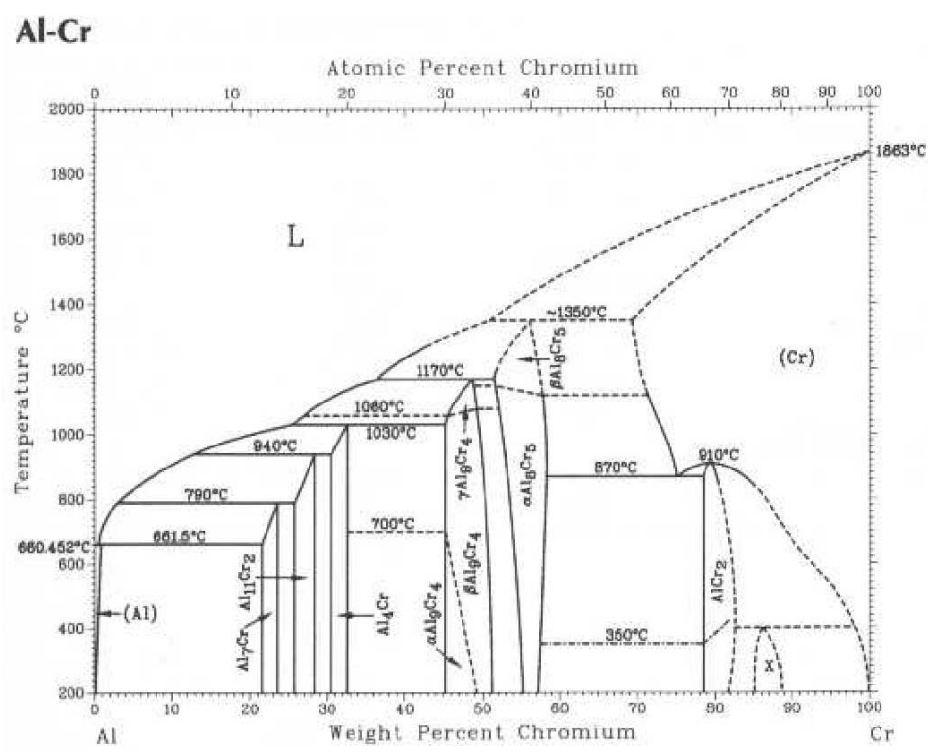

Figura 6.24: Diagrama de fases do sistema Alumínio-Cromo obtido do ASM Metals Handbook-Volume 3-Alloy Phase Diagrams.

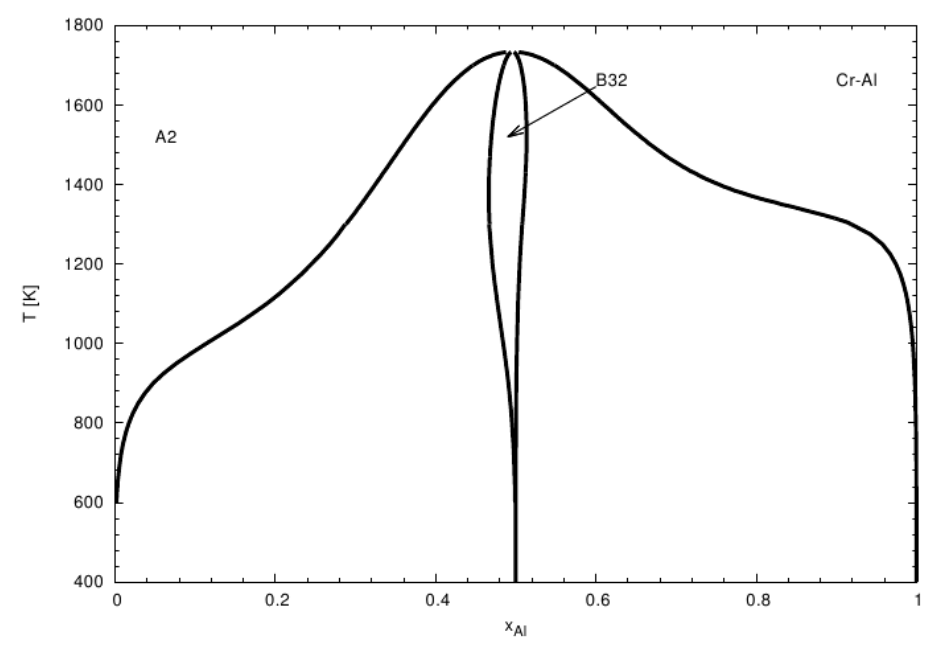

Figura 6.25: Diagrama de fases do sistema Alumínio-Cromo obtido neste trabalho.

alizaram uma reavaliação por meio de técnicas de cálculos de diagramas de fases (CALPHAD) do sistema Al-Cr e em nenhum momento consideraram a presença de uma fase ordenada. Um resultado recente [115] sugere que a presença de uma fase B32 estável no sistema Al-Cr não é uma ocorrência fortuíta, mas sim uma tendência observada em outras ligas Al-M com M 
pertencendo aos grupos VB e VIB da tabela periódica. 


\section{Capítulo 7}

\section{Resultados e \\ Discussões: 04- Sistema Ternário Fe-Cr-Al}

\subsection{Sistema Ternário Fe-Cr-Al}

Para fazer o Diagrama de Fases do sistema ternário são necessário 21 compostos, como foi discutido no capitulo III. Em cada sistema binário temos quatro compostos: B2, B32 e duas versões de $\mathrm{D0}_{3}$. Como são três sistemas binários teremos doze compostos; se adicionarmos os compostos A2 obtêm-se quinze casos correspondentes aos binários, os quais já foram calculados e analisados, resultando em um conjunto de parâmetros de interação para o CVM, com os quais descreve-se os três binários. Na Tabela 7.1 As seis configurações

\begin{tabular}{cccc}
\hline Parâmetros I. CVM & FeAl & Fecr & AlCr \\
\hline \hline$w_{A B}^{1}$ & -1064.3 & +552.9 & -214.25 \\
$w_{A B}^{2}$ & +757.91 & +203.3 & -16.82 \\
$w_{A B A B}$ & -502.2 & -129.46 & -147.69 \\
$W_{A B B B}$ & -261.645 & -122.62 & +212.39 \\
\hline
\end{tabular}

Tabela 7.1: Parâmetros de interação do CVM dos sistemas binários com polarização de spin para o caso FPLAPW-GGA[48], dados em $k_{B} K$.

remanescentes são características do sistema ternário propriamente dito. Estas configurações se agrupam duas a duas. Os grupos assim formados diferem apenas pela permutação das três espécies atômicas. Podemos, portanto, analisar as estruturas concentrando-nos em apenas um dos grupos. Para este fim 
iremos estudar as duas fases com estequiometria $\mathrm{Fe}_{2} \mathrm{AlCr}$, denominadas respectivamente $L 2_{1}$ e $\mathrm{F} 43 \mathrm{~m}$.

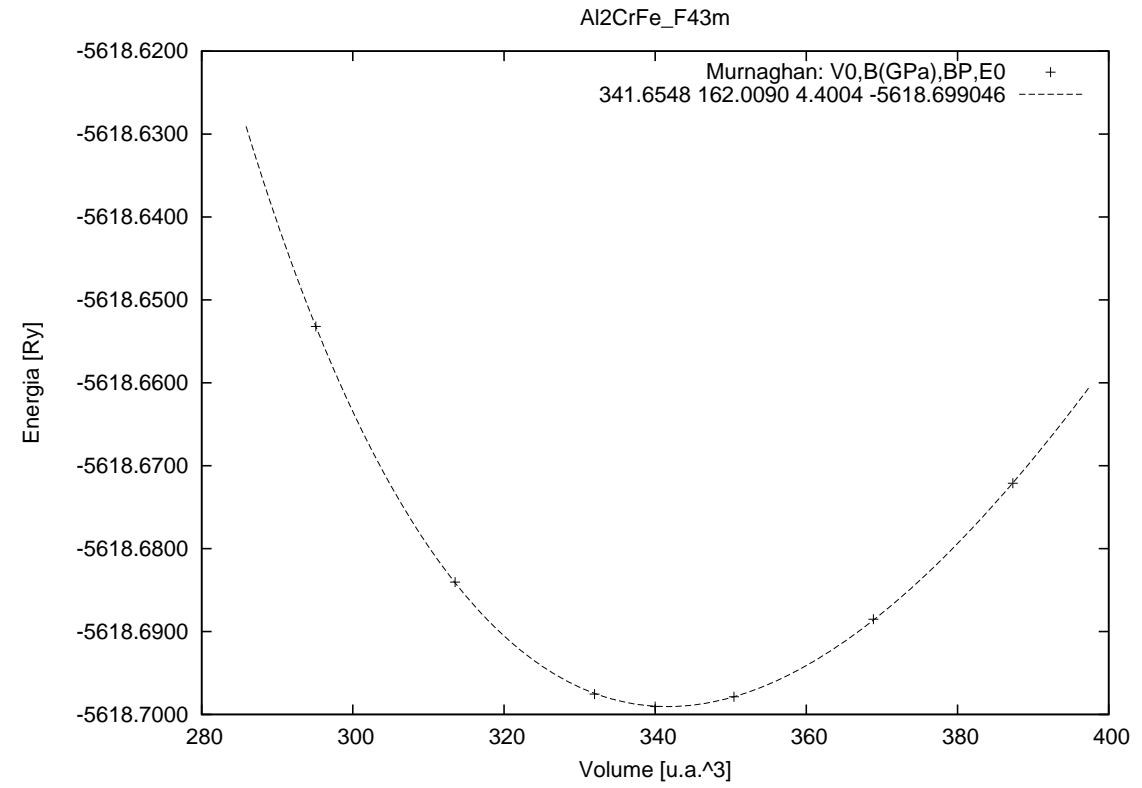

Figura 7.1: Otimização estrutural da liga Ternária Alumínio-Cromo-Ferro (Al2CrFe-F43m) para o caso FP-LAPW. Na legenda temos: $V_{0}$ é o volume mínimo em unidades atômicas; $\mathrm{B}(\mathrm{GPa})$ é o Bulk módulo, BP é o Derivada do Bulk Módulo e $E_{0}$ a energia mínima. 


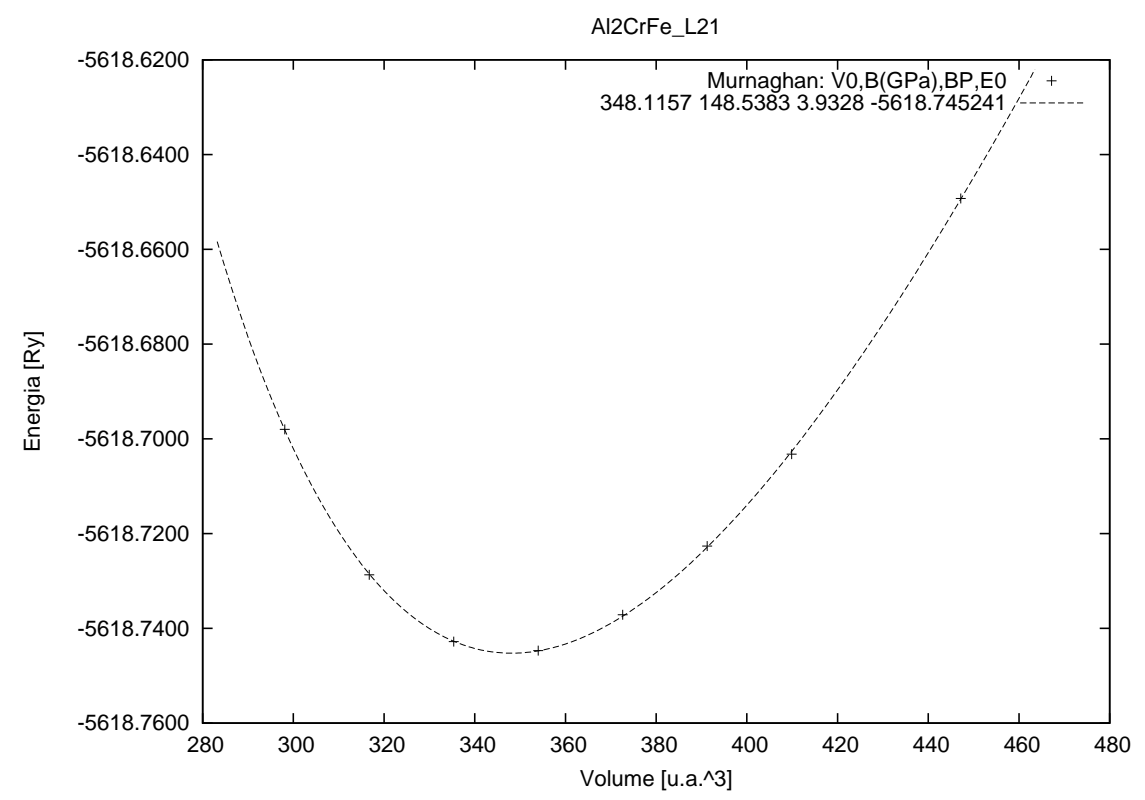

Figura 7.2: Otimização estrutural da liga Ternária Alumínio-Cromo-Ferro (Al2CrFe-L21) para o caso FP-LAPW. Na legenda temos: $V_{0}$ é o volume mínimo em unidades atômicas; $\mathrm{B}(\mathrm{GPa})$ é o Bulk módulo, BP é o Derivada do Bulk Módulo e $E_{0}$ a energia mínima.

Na Tabela 7.2 apresentamos os resultados $E_{T}$ dos seis compostos do sistema ternário, assim como os valores dos parâmetros convergidos dentro do código WIEN2K com o método FPLAPW-GGA [48]. 


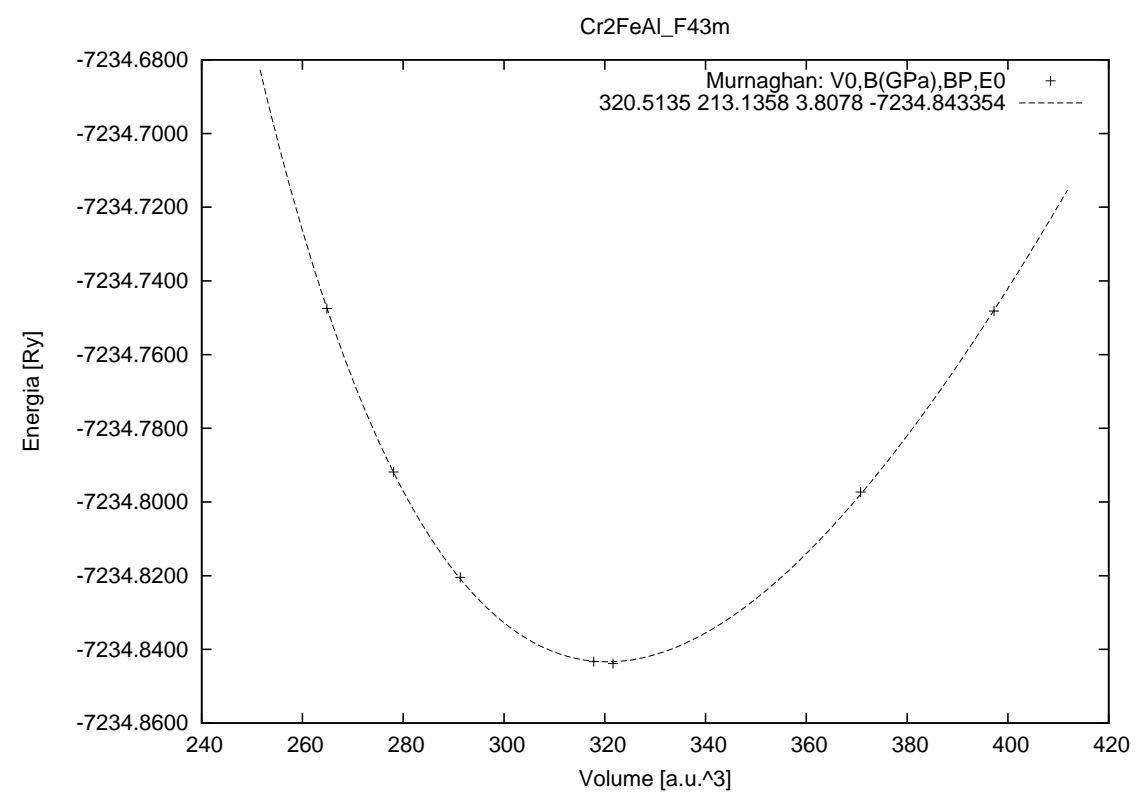

Figura 7.3: Otimização estrutural da liga Ternária Alumínio-Cromo-Ferro (Cr2FeAl-F43m) para o caso FP-LAPW. Na legenda temos: $V_{0}$ é o volume mínimo em unidades atômicas; $\mathrm{B}(\mathrm{GPa})$ é o Bulk módulo, BP é o Derivada do Bulk Módulo e $E_{0}$ a energia mínima.

Tabela 7.2: Energia total calculada para os seis compostos formando a base para a expansão de cluster aplicada ao sistema Fe-Cr-Al utilizando todos os parâmetros de controle otimizados. Para todos os calculos Rk$\max =\mathrm{Lmax}=10$, número de pontos $\mathrm{K}$ na zona irredutível de Brillouin foi de 286. O momento magnético médio no átomo de Ferro (MM) é apresentado (em magneton de Bohr, $\mu_{B}$ ). Os parâmetros de rede são expressados em nm e os valores da das energias são representados em Rydberg (Ry) por formula unitária $\left(1 \mathrm{Ry}=2.1798741 \times 10^{-18} \mathrm{~J}\right)$.

\begin{tabular}{clcccc}
\hline Calculo & Estequiometria & Estrutura & $a_{0}$ & $\mathrm{MM}$ & $E_{T}$ \\
\hline Ferromagnético & $\mathrm{Fe}_{2} \mathrm{AlCr}$ & $\mathrm{L} 2_{1}$ & 0.565 & 0.21 & -7678.696212 \\
& $\mathrm{Fe}_{2} \mathrm{AlCr}$ & $\mathrm{F} 43 \mathrm{~m}$ & 0.577 & 1.98 & -7678.675057 \\
& $\mathrm{Al}_{2} \mathrm{FeCr}$ & $\mathrm{L} 2_{1}$ & 0.591 & +0.61 & -5618.745312 \\
& $\mathrm{Al}_{2} \mathrm{FeCr}$ & $\mathrm{F} 43 \mathrm{~m}$ & 0.587 & 0.68 & -5618.699244 \\
& $\mathrm{Cr}_{2} \mathrm{FeAl}$ & $\mathrm{L} 2_{1}$ & 0.586 & 1.09 & -7234.798387 \\
& $\mathrm{Cr}_{2} \mathrm{FeAl}$ & $\mathrm{F} 43 \mathrm{~m}$ & 0.575 & 0.66 & -7234.843974 \\
\hline
\end{tabular}

Na tabela 7.3 apresentamos a energia de formação por átomo dos seis compostos do sistema ternário. Observa-se que o composto $\mathrm{L2}_{1}\left(\mathrm{Al} \mathrm{l}_{2} \mathrm{FeCr}\right)$ 


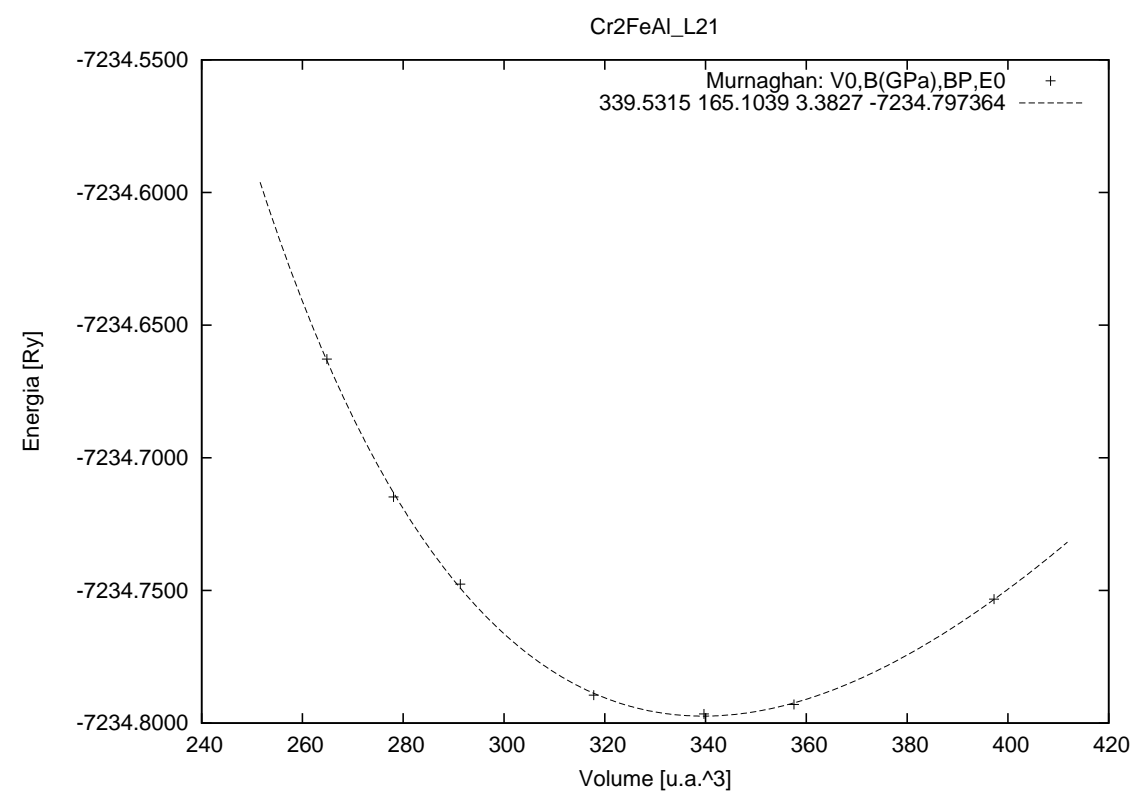

Figura 7.4: Otimização estrutural da liga Ternária Alumínio-Cromo-Ferro (Cr2FeAl-L21) para o caso FP-LAPW. Na legenda temos: $V_{0}$ é o volume mínimo em unidades atômicas; $\mathrm{B}(\mathrm{GPa})$ é o Bulk módulo, BP é o Derivada do Bulk Módulo e $E_{0}$ a energia mínima.

tem a mais baixa energia de formação no estado fundamental dentre todos os compostos estudados neste trabalho, indicando uma forte estabilização desta fase no sistema. Este resultado terá fortes implicações sobre o diagrama de fases calculado e irá ser discutido em detalhe mais adiante. Uma análise do estado fundamental do sistema ternário também é possível [119], porém a utilidade de um diagrama de estado fundamental ( como os das figuras $6.6,6.14,6.23)$ fica diminuída em um sistema ternário devido a três razões principais:

- O caráter tridimensional dos diagramas dificulta sua visualização,

- os cálculos de diagramas ternários são feitos em seções isotérmicas e

- os sistemas binários são casos limites do sistema ternário quando um dos componentes fica infinitamente diluído.

Estas duas últimas razões, em particular, reduzem a importância do estado fundamental, pois as temperaturas dos cálculos são geralmente muito maiores que 0 (zero) Kelvin e as informações dos cálculos binários podem ser usadas como ponto de partida para o cálculo ternário, assim como o estado fundamental é usado no caso binário. Este ponto voltará a ser discutido em detalhe 


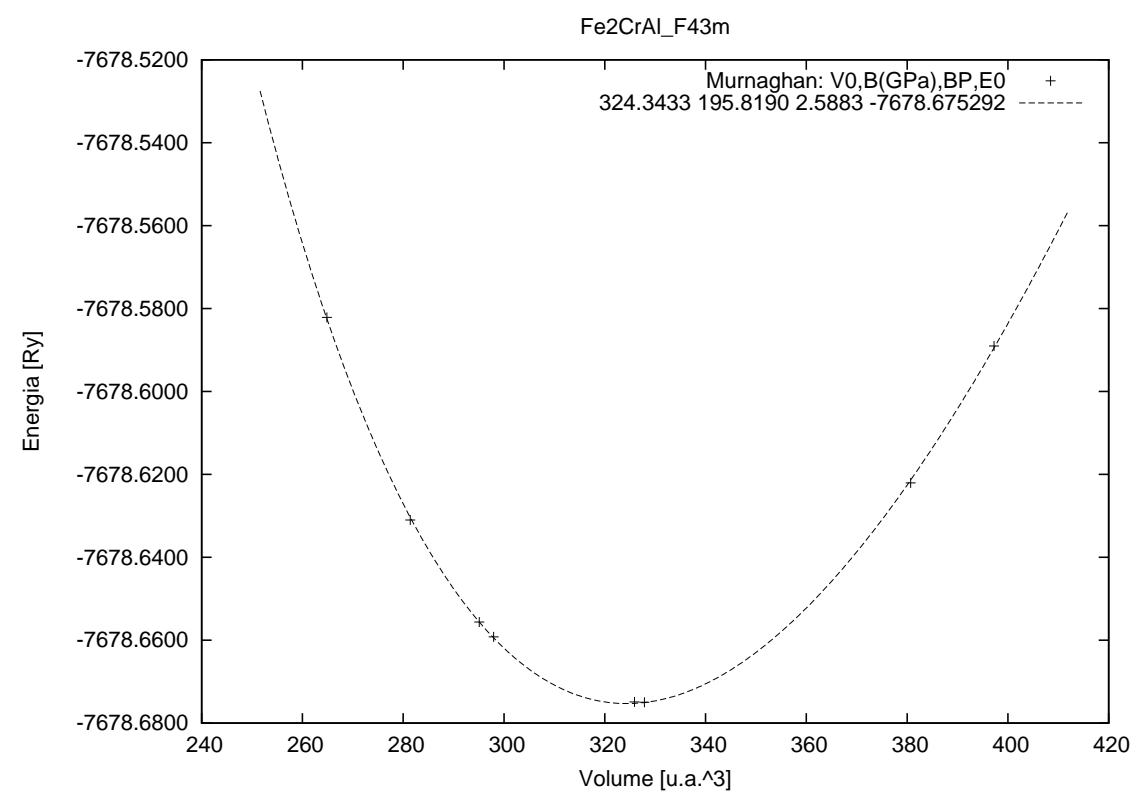

Figura 7.5: Otimização estrutural da liga Ternária Alumínio-Cromo-Ferro (Fe2CrAl-F43m) para o caso FP-LAPW. Na legenda temos: $V_{0}$ é o volume mínimo em unidades atômicas; $\mathrm{B}(\mathrm{GPa})$ é o Bulk módulo, BP é o Derivada do Bulk Módulo e $E_{0}$ a energia mínima.

mais adiante.

Na tabela 7.4 estão os dados de entrada para o CVM correspondente as seis

\begin{tabular}{ccccc}
\hline Sistema & Fase & $\mathrm{Ry}$ & $\mathrm{kJ} / \mathrm{mol}$ & $K_{B} K$ \\
\hline \hline $\mathrm{Fe}_{2} \mathrm{CrAl}$ & $\mathrm{L} 2{ }_{1}$ & -0.0124 & -16.377 & -1969.72 \\
$\mathrm{Fe}_{2} \mathrm{CrAl}$ & $\mathrm{F} 43 \mathrm{~m}$ & -0.0072 & -9.434 & -1134.66 \\
$\mathrm{Al}_{2} \mathrm{FeCr}$ & $\mathrm{L} 2{ }_{1}$ & -0.0185 & -24.335 & -2926.74 \\
$\mathrm{Al}_{2} \mathrm{FeCr}$ & $\mathrm{F} 43 \mathrm{~m}$ & -0.0070 & -9.215 & -1108.29 \\
$\mathrm{Cr}_{2} \mathrm{FeAl}$ & $\mathrm{L} 2{ }_{1}$ & +0.0050 & +6.592 & +792.86 \\
$\mathrm{Cr}_{2} \mathrm{FeAl}$ & $\mathrm{F} 43 \mathrm{~m}$ & -0.0064 & -8.369 & -1006.61 \\
\hline
\end{tabular}

Tabela 7.3: Energia de formação do sistema Fe-Cr-Al com polarização de spin e sem acoplamento spin orbita, utilizando o método FP-LAPW.

fases do sistema ternário. Observa-se que o composto $\mathrm{Fe}_{2} \mathrm{Cr} \mathrm{Al}$, na configuração $L 2_{1}$ corresponde ao parâmetro de interação de cluster mais negativo de todo o sistema. 


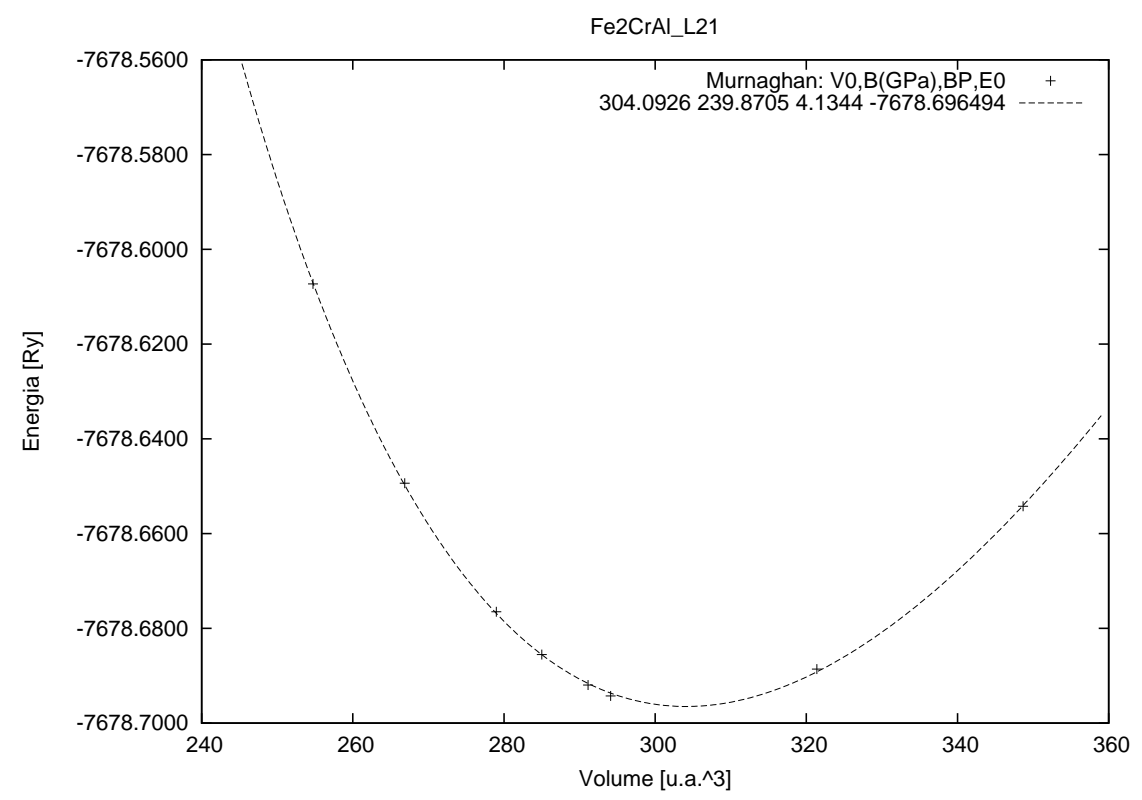

Figura 7.6: Otimização estrutural da liga Ternária Alumínio-Cromo-Ferro (Fe2CrAl-L21) para o caso FP-LAPW. Na legenda temos: $V_{0}$ é o volume mínimo em unidades atômicas; $\mathrm{B}(\mathrm{GPa})$ é o Bulk módulo, BP é o Derivada do Bulk Módulo e $E_{0}$ a energia mínima.

\begin{tabular}{rcll}
\hline Estequiometria & Estrutura & Energia de formação & p. interação de cluster $\left[k_{B} \mathrm{~K}\right]$ \\
\hline $\mathrm{Fe}{ }_{2} \mathrm{AlCr}$ & $\mathrm{L} 2_{1}$ & -16.38 & $\tilde{w}_{F e, F e, A l, C r}=-382.9$ \\
$\mathrm{FeAlFeCr}$ & $\mathrm{F} 4 \overline{3} \mathrm{~m}$ & -9.43 & $\tilde{w}_{F e, A l, F e, C r}=-345.4$ \\
$\mathrm{Al}_{2} \mathrm{CrFe}$ & $\mathrm{L} 2_{1}$ & -24.34 & $\tilde{w}_{A l, A l, C r, F e}=-111.8$ \\
$\mathrm{AlCrAlFe}$ & $\mathrm{F} 4 \overline{3} \mathrm{~m}$ & -9.22 & $\tilde{w}_{A l, C r, A l, F e}=-217.8$ \\
$\mathrm{Cr}_{2} \mathrm{FeAl}$ & $\mathrm{L} 2_{1}$ & +6.60 & $\tilde{w}_{C r, C r, F e, A l}=+90.9$ \\
$\mathrm{CrFeCrAl}$ & $\mathrm{F} 4 \overline{3} \mathrm{~m}$ & -8.37 & $\tilde{w}_{C r, F e, C r, A l}=-324.3$ \\
\hline
\end{tabular}

Tabela 7.4: Parâmetros de interação do cluster em unidades de constante de Boltzmann $\left(1 k_{B} \mathrm{k}=8.3145 \mathrm{~J} \mathrm{~mol}^{-1}\right)$.

A representação gráfica de um diagrama de fase ternário é dificultada pela sua característica tridimensional. Tradicionalmente representa-se um diagrama de fase ternário por meio de seções bidimensionais do diagrama completo. Neste trabalho iremos utilizar unicamente seções isotérmicas, sendo que a temperatura dos cálculos será variada para visualizar a estrutura tridimensional do diagrama. A representação gráfica da seção isotérmica de um diagrama ternário é feita com o auxilio do chamado "triângulo de Gibbs" para respeitar a dependência entre as três variáveis de composição 


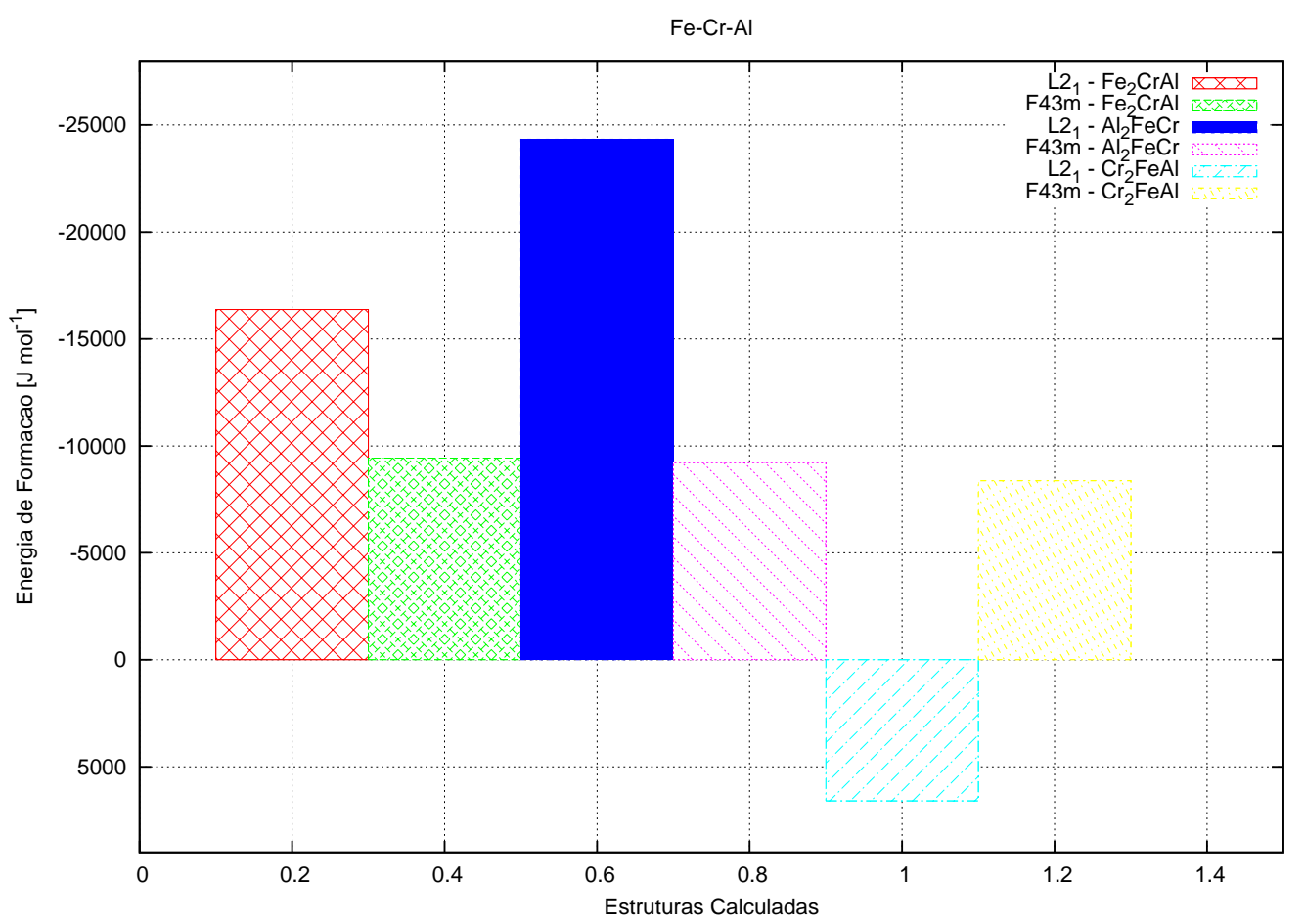

Figura 7.7: Energia de formação dos compostos usados para construir a base para a expansão de cluster do sistema $\mathrm{Fe}-\mathrm{Cr}-\mathrm{Al}$.

$\left(x_{F e}+x_{A l}+x_{C r}=1\right)$. Cada vértice representa uma estrutura A2 e cada lado do triângulo representa um sistema binário, que já foi estudado. A composição de cada elemento ( $\mathrm{Fe}, \mathrm{Cr}, \mathrm{Al}$ ) vai ser 1.0 (ou 100\%) no vértice corresponde e $0 \%$ no lado oposto [120]. A leitura das concentrações em um triângulo de Gibbs é feita com o auxilio de linhas guias. A linha guia usada para ler as coordenadas é indicada pela inclinação dos marcos de escala no respectivo eixo. O próximo passo do trabalho é usar os parâmetros de interação calculados e montar as seções isotermicas do diagrama de fase ternário Fe-Cr-Al. 


\section{Capítulo 8}

\section{Sumário}

Neste trabalho apresentamos um trabalho interdisciplinar unindo três áreas do conhecimento, a Mecânica Quântica, Mecânica Estatística e Engenharia Metalúrgica para descrever propriedades macroscópicas a partir de informações da estrutura atômica do material de interesse. Para isso utilizamos a chamada termodinâmica computacional ab-initio, ou seja, usamos o método ab-initio para calcular a energia volumétrica no estado fundamental dos sistemas que nos interessam e o método de variação de clusters (CVM) para construir a termodinâmica do sistema. Utilizamos como método abinitio embebido no código WIEN2K. De forma sucinta podemos descrever este trabalho como o uso de cálculos ab-initio de estrutura eletrônica em conjunto com o método variacional de clusters para obter propriedades termodinâmicas para os sistemas Fe-Cr-Al na estrutura cúbica de corpo centrado. Para isso usamos o método FP-LAPW para o cálculo de energia total volumétrica em sistemas metálicos e o CVM na aproximação do tetraedro irregular para construir a termodinâmica do sistema. Primeiramente definimos os critérios de otimização para as estruturas de referência, escolhemos trabalhar com os elementos unários Fe BCC, Al BCC e Cr BCC. Era de nosso conhecimento que o Cr BCC é antiferromagnético, mas realizamos os cálculos ab-initio procurando a estrutura mais estável e chegamos que o estado fundamental dele realmente é o que comprova os resultados da literatura descrito por Cottenier e autores para o estado fundamental do Cr. Definimos quais eram os parâmetros de controle que deveriamos adotar para obter o estado fundamental nos cálculos de estrutura eletrônica com o WIEN2K. Com o conhecimento dos parâmetros de controle para a convergência fomos analisar a influência das premissas básicas possíveis de serem utilizadas com o código WIEN2K, para isso escolhemos um sistema que já havia sido estudado na literatura. Concluímos que um cuidado especial deve ser tomado quanto a determinação precisa de detalhes com relação aos cantos do diagrama de fases 
(fases ricas no elemento unário). Como já se sabe, estes deve ser exclusivo (e claramente definidos) no decurso de um determinado cálculo. Nosso resultados também mostra que a escolha do termo de troca e correlação é importante e, não era tão esperado para esses (que não são tão pesado) compostos intermetálicos d, a inclusão dos efeitos relativísticos pode mudar a determinação de detalhes finos do diagrama de fases. O sistema Fe-Al foi também revisitado com a inclusão de efeitos relativísticos. Como consequëncia, a energia de formação do composto B2-FeAl tornou-se mais negativo quando comparada com os resultados anteriores conhecidos da literatura [109], o que atualmente implica em uma melhor concordância com dados calorimetricos experimentais. Esta mudança na energia de formação afeta muito pouco no diagrama de fases calculado Fe-Al BCC, em particular sua topologia mantêm-se inalterada. O sistema Al-Cr foi calculado pela primeira vez utilizando um procedimento completamente ab-initio, há muita discussão na literatura quanto a existência de uma fase experimental ordenada. Há controvérsia se a fase indentificada nos diagramas de fases CALPHAD quando ocorre uma transição de segunda-ordem do $\mathrm{Cr} \mathrm{BCC}$ para o $\mathrm{AlCr} \mathrm{CsCl}$ se é realmente o tipo B2 ou B32. O método CALPHAD não permite determinar tal fase ao passo que nossa metodologia permitiu definir esta fase como sendo a B32. Para o sistema $\mathrm{Fe}-\mathrm{Cr}$, nosso resultado mostrou uma excelênte concordância com relação a topologia do diagrama de fase experimental, o que sugere que a discussão na literatura sobre o corner rico em ferro[123] deve-se manter o diagrama de fase experimental atual pois o limite de solubilidade descrito pelo diagrama de fase experimental é descrito pelo diagrama de fases ab-initio que foi calculado pela primeira vez com está metodologia. O grande desafio dos sistemas $\mathrm{Fe}-\mathrm{Cr}$ e Al-Cr está no comportamento magnético bastante intrincado que estes apresenta e na dificuldade de definir quais são suas estruturas estáveis, todos os resultados apresentados já são para a estrutura magneticamente mais estável. Para o sistema ternário, fizemos a otimização estrutural e encontramos as seis estruturas mais estáveis. Obtivemos as energias de formação para cada estrutura onde foi possível observar que a única estrutura que contribui para o desordenamento é o $\mathrm{Cr}_{2} \mathrm{FeAl} \mathrm{L} 2_{1}$. Os parâmetros de interação já estão calculados e os diagramas de fases em seções isotermicas para os sistemas ternários são as próximas etapas do trabalho. 


\section{Apêndice: A - Propriedades Locais}

\subsubsection{Parâmetros hiperfinos}

Podemos dividir o estudo das propriedades dos materiais em duas grandes classes: propriedades de volume ("bulk") e propriedades locais. Esta divisão não é de forma alguma rigorosa, já que, certamente, as propriedades de volume são resultados das somas das diversas contribuições locais, porém acredito que facilite uma primeira grande visão da área. Por exemplo, técnicas experimentais, que permitem a medida de magnetização em uma dada amostra, nos fornecem informações sobre o volume como um todo. Por outro lado, o estudo de propriedades locais, isto é, propriedades dependentes de cada sítio atômico, podem, por exemplo, ser realizado através de técnicas que explorem a interação entre um dado núcleo atômico e suas vizinhanças. Estas são as chamadas interações hiperfinas. De forma interdisciplinar, a área de interações hiperfinas, entrelaça e se constitui em uma das regiões de fronteira entre as grandes áreas de física nuclear e de física de materiais. Embora esta não seja uma nova área de pesquisa, com a crescente sofisticação das técnicas de produção de feixes iônicos e de análise durante a ocorrência dos próprios processos, cada vez mais pesquisadores em diversos centros com aceleradores nucleares de diversos portes, encontram na investigação das interações hiperfinas uma poderosa ferramenta. Neste sentido, técnicas experimentais já bem estabelecidas, tais como Ressonância Magnética Nuclear (NMR), Ressonância de Quadrupolo Nuclear (NQR), Correlação Angular Perturbada TempoDiferencial (TDPAC), Distribuição Angular Perturbada Tempo-Diferencial (TDPAD), Espectroscopia Mössbauer, etc., tem sido largamente utilizadas para nos fornecer informações sobre as distribuições eletrônicas locais. O conhecimento da distribuição espacial dos átomos em um sólido desordenada (mesmo com pequeno grau de desordem) é, até hoje, bastante limitado, já que as técnicas experimentais usuais de difração não nos fornecem informações sobre as características direcionais da coordenação atômica de curto 
alcance. È por isso que tem havido crescente interesse no aprimoramento e sofisticação das técnicas que permitem caracterizar vizinhanças locais como é o caso das que se utilizam de interação hiperfina. Porem a informação experimentalmente acessível, neste caso, é trazido como um produto entre uma quantidade característica nuclear e uma quantidade extra-nuclear presente no núcleo. A quantidade extra-nuclear é objeto do cálculo de estrutura eletrônica e aquelas que são tratadas usualmente são o Gradiente de Campo Elétrico (GCE), o Campo Magnético Hiperfino (HFF) e o Deslocamento IsoméricoAlCr-Phase (IS). Estas três quantidades, que também são chamadas de "parâmetros hiperfinos", nos podem então fornecer informações sobre a distribuição eletrônica local em um dado sólido. A avaliação teórica do GCE, HFF, IS é, portanto, interessante já que pode ajudar tanto na interpretação de resultados experimentais como na estimativa da adequação dos modelos estruturais para sistemas com quebra de periodicidade. Segundo a experiência acumulada na literatura para sistemas metálicos, é geralmente assumido que o termo usualmente dominante para o campo magnético hiperfino é dado pelo termo de contato de Fermi. Este termo é determinado, basicamente, pela densidade de magnetização na posição do núcleo em consideração. Além desta, existem contribuições dipolar e orbital para o campo hiperfino, que normalmente são assumidas como de menor importância. Chamaremos no presente texto, de forma um pouco abusiva, porém usual na literatura, de campo magnético hiperfino (HFF) a contribuição de contato de Fermi para o campo hiperfino, fazendo referência explícita as outras contribuições quando necessário. Porém, no presente texto restringimos ao estudo do campo hiperfino em sólidos metálicos, em particular contendo metais de transição.

Detalhes da implementação do cálculo do Campo hiperfino, bem como da implementação do cálculo do acoplamento spin-orbita no código WIEN2K pode ser encontrado no artigo do P. Novák [121] e [122]. O valor do Campo Hiperfino (HFF) para o sistema Fe-Al para os cálculos efetuados com polarização de spin, são mostrados na Tabela 8.2. 


\begin{tabular}{cccccc}
\hline Sistema & Fase & $\mathrm{Z}_{N N}$ & $\mathrm{~d}_{N N}$ & $\mathrm{Z}_{2 N N}$ & $\mathrm{~d}_{2 N N}$ \\
\hline \hline $\mathrm{Fe}$ & $\mathrm{A} 2$ & $8 \mathrm{Fe}$ & 0.247 & $6 \mathrm{Fe}$ & 0.285 \\
$\mathrm{Al}$ & $\mathrm{A} 2$ & $8 \mathrm{Al}$ & 0.280 & $6 \mathrm{Al}$ & 0.323 \\
$\mathrm{FeAl}$ & $\mathrm{B} 2$ & $8 \mathrm{Al}$ & 0.248 & $6 \mathrm{Fe}$ & 0.287 \\
$\mathrm{FeAl}$ & $\mathrm{B} 32$ & $4 \mathrm{Fe}$ e $4 \mathrm{Al}$ & 0.254 & $6 \mathrm{Al}$ & 0.293 \\
$\mathrm{Fe} 3 \mathrm{Al}$ & $\mathrm{D} 03$ & $8 \mathrm{Fe}$ & 0.248 & $6 \mathrm{Fe}$ & 0.286 \\
$\mathrm{FeAl} 3$ & $\mathrm{D} 03$ & $8 \mathrm{Al}$ & 0.259 & $6 \mathrm{Fe}$ & 0.299 \\
\hline
\end{tabular}

Tabela 8.1: Distância obtida da primeira e segunda vizinhança do sistema $F e-A l$ ferromagnético, utilizando o método FP-LAPW. Onde: $d_{N N}=$ distância do primeiro vizinho (em $\mathrm{nm}$ ) e $Z_{N N}$ é o átomo na primeira vizinhança $e$ $d_{2 N N}, Z_{2 N N}$ são as distâncias (em nm) e átomos na segunda vizinhan $\tilde{A} \S a$.

\begin{tabular}{ccccc}
\hline Sistema & Fase & Valência & Core & Total \\
\hline \hline Fe & A2 & 281.938 & -597.485 & -315.546 \\
Al & A2 & $1.10^{-3}$ & 0.001 & $3.10^{-3}$ \\
FeAl-Fe & B2 & +107.538 & -181.641 & -74.103 \\
FeAl-Al & B2 & -38.731 & 0.042 & -38.688 \\
FeAl-Fe & B32 & +45.881 & -249.845 & -203.965 \\
FeAl-Al & B32 & +2.340 & 0.834 & +3.174 \\
Fe3Al-Fe1 & D03 & -7.066 & -304.757 & -311.823 \\
Fe3Al-Fe2 & D03 & -17.253 & -238.971 & -256.224 \\
Fe3Al-Al & D03 & -43.095 & +0.925 & -42.169 \\
FeAl3-Fe & D03 & +0.014 & -0.006 & +0.008 \\
FeAl3-Al1 & D03 & -0.002 & 0.000 & -0.003 \\
FeAl3-Al2 & D03 & 0.000 & -0.001 & -0.001 \\
\hline
\end{tabular}

Tabela 8.2: Campo Hiperfino(HFF) para o sistema Fe-Al, calculo ferromagnético com polarização de spin e sem acoplamento spin-orbita, campo hiperfino dado em KGauss. 


\begin{tabular}{ccccc}
\hline Sistema & Fase & Valência & Core & Total \\
\hline \hline Fe & A2 & 308.41 & -607.65 & -299.24 \\
Cr & A2 & $-4.10^{-3}$ & $+4.10^{3}$ & 0 \\
FeCr-Fe & B2 & +277.30 & -375.60 & -98.30 \\
FeCr-Cr & B2 & +3.04 & -108.06 & -105.03 \\
FeCr-Fe & B32 & +86.92 & -231.46 & -144.54 \\
FeCr-Cr & B32 & -68.87 & -6.98 & -75.85 \\
Fe3Cr-Fe1 & D03 & +324.53 & -508.95 & -184.42 \\
Fe3Cr-Fe2 & D03 & +424.45 & -608.54 & -184.09 \\
Fe3Cr-Cr & D03 & -204.54 & +75.92 & -128.62 \\
FeCr3-Fe & D03 & +335.18 & -448.27 & -113.09 \\
FeCr3-Cr1 & D03 & -35.26 & -24.83 & -60.09 \\
FeCr3-Cr2 & D03 & +59.89 & -13.33 & +46.56 \\
\hline
\end{tabular}

Tabela 8.3: Campo Hiperfino(HFF) para o sistema Fe-Al, calculo ferromagnético com polarização de spin e sem acoplamento spin-orbita, campo hiperfino dado em KGauss. 


\section{Referências Bibliográficas}

[1] Pablo Guillermo Ormeño-Gonzales. Tese de doutoramento: Determinação dos diagramas de fases do sistema Fe-Al-Mo cúbico de corpo centrado por cálculos de primeiros princípios. 2002.

[2] R. J. Van Thyne, H. D. Kessler and M. Hansen. Transactions of American Society for Metals, 44, 974-989, 1952.

[3] Kurt Binder, Monte Carlo Methods in Statistical Physics. vol.7 of topics in Current Physics, Springer, Berlin, 1986.

[4] Larry Kaufman e Harold Bernstein. Computer Calculation of Phase Diagrams, Academics Press New York and London, 1970.

[5] N. Saunders e A. P. Miodiownik, CALPHAD - Calculations of Phase Diagrams, Pergamon Press New York, ISBN: 0080421296, 1998.

[6] C. E. Dahmani, M. C. Cadeville, J. M. Sanchez e J. L. MoranLopez. Physical Review Letters, 55, 1208-1211, 1985.

[7] W. L. Bragg e E. J. Williams. Proceedings of the Royal Society of London, 145-A, 699-730, 1934.

[8] W. L. Bragg e E. J. Williams. Proceedings of the Royal Society of London, 151-A, 540-566, 1935.

[9] N. Dupin, I. Ansara e B. Sundman. CALPHAD, 25, 279-298, 2001.

[10] K. Hack. The SGTE Casebook Thermodinamics at Work. Materials Modelling Series. The Institute of Materials, 1996.

[11] N. A. Gokcen. Journal de Chimie Physique et de Physico-Chimie Biologique, 94, 817-843, 1997.

[12] W. A. Oates, H. Wenz e T. Mohri. CALPHAD, 20, 37-45, 1996. 
[13] D. de Fontaine. Solid State Physics, 47, 133-176. 1994.

[14] M. Sluiter, D. de Fontaine, X. Q. Guo, R. Podloucky e A. J. Freeman. Physical Review B, 42, 10460-10476, 1990.

[15] Mark Asta, Didier de Fontaine, Mark van Schilfgaarde, Marcel Sluiter e Michael Methfessel. Physical Review B, 5055-5072, 1992.

[16] R. Kainuma, M. Palm. e G. Inden, Intermetallics, 2, 321-332, 1994.

[17] Mark Asta, Didier de Fontaine e Mark Van Schilfgaarde. Journal of Materials Research, 8, 2554-2568, 1993.

[18] A. Pasturel, C. Colinet, A. T. Paxton e M. van Schilfgaarde. Journal of Physics: Condensed Matter, 4, 945-959, 1992.

[19] D. B. Miracle. Acta Metallurgica et Materialia, 41, 649-684, 1993.

[20] Catherine Colinet e Alain Pasturel. Zeitschrift für Metallkunde, 89, 863867, 1998.

[21] F. Lechermann e M. Fähnle. Physical Review B, 63, 012104 (1-4), 2001.

[22] Kurt Binder, Joel L. Lebowitz, Mohan K. Phani e Marvin H. Kalos. Acta Metallurgica, 29,1655-1665, 1981.

[23] Burkhard Dünweg and Kurt Binder. Physical Review B, 36, 69356952, 1987.

[24] H. Ackermann, G. Inden e R. Kikuchi. Acta Metallurgica, 37, 1-7, 1989.

[25] Cláudio Geraldo Schön e Gerhard Inden. Computational Materials Science, 20, 98-106, 2001.

[26] Luiz G. Ferreira, C. Wolverton e Alex Zunger. Journal of Chemical Physics. 108, 2912-2918, 1998.

[27] P. E. A. Turchi, Larry Kaufmann e Zi-Kiu Liu. CALPHAD approach to stability and aging of candidate alloy for the Yucca Mountain Project. $30^{\text {th }}$ meeting of Calphad. $27^{\text {th }}$ May - $1^{\text {st }}$ June, 2001. York England, UK.

[28] S. C. Deevi, V. K. Sikka e C. T. Liu. Progress in Materials Science, 42, 177-192, 1997.

[29] C. T. Liu, J. Stringer, J. N. Mundy, L. L. Horton e P. Angelini. Intermetallics, 5, 579-596, 1997. 
[30] Cláudio Geraldo Schön. Texturas e relações de orientação, deformação plástica, recristalização, crescimento de grãos. Ed. Padilha A.F., Tschiptsohn A. P., Schön C. G., Landgrof F. J., Goldstein H., Falleiros I. G. S., De Lima N. B.. Editora EPUSP, 255-272, São Paulo, 2001.

[31] I. Baker e P. R. Munroe. International Materials Review, 42, 181205. 1997.

[32] Neil W. Aschcroft e N. David Mernim. Solid State Physics. Saunders College Publishers, 1976.

[33] Gehrard Inden e Werner Pepperhoff. Zeitschrift für Metallkunde, 81, 770-773, 1990.

[34] Parr G. Robert e W. Yang. 47-66. Oxford University Press, 1998.

[35] Adalberto Fazzio, Estrutura Eletrônica de Materiais. Notas de aula. Universidade Federal de Santa Maria, 2000.

[36] P. Hohenberg e W. Kohn. Physical Review, 136B, 864-871, 1964.

[37] W. Kohn and L. J. Sham. Physical Review, 140A, 1133-1138, 1965.

[38] David J. Singh. Planewaves, Pseudopotentials and the LAPW method. Naval Research Laboratory. Washington, DC, 1994.

[39] M. Weinert, E. Wimmer e A. J. Freeman. Physical Review B, 26, 4571$4578,1982$.

[40] Bernd Kohler, Steffen Wilke, Mathias Scheffler, Robert Kouba e Claudia Ambrosch-Draxl. Computer Physics Communications, 94, 31-48, 1996.

[41] K.Capelle, Brazilian Journal of Physics, 36, 4A, 1318- 1343, 2006.

[42] L. Hedin e B. I. Lundqvist. Journal of Physics C: Solid State Physics, 4, 2064-2083, 1971.

[43] V. L. Moruzzi, J. F. Janak e A. R. Williams. Calculated Properties of Metals, Pergamon, NY, 1978.

[44] John P. Perdew e Wang Yue. Physical Review B, 45, 13244-13249, 1992.

[45] John P. Perdew e Wang Yue. Physical Review B, 33, 8800-8802, 1986.

[46] A. D. Becke. Physical Review A, 38, 3098-3100, 1988. 
[47] E. Engel e S. H. Vosko. Physical Review B, 47, 13164-13174, 1993.

[48] John P. Perdew, Kieron Burke e Matthias Ernzerhof. Physical Review Letters, 77, 3865-3868, 1996.

[49] Ney Sodré, Joelson C. Garcia, Lucy V. Assali, Pablo G. Gonzales Ormeño, Peter Blaha, Helena M. Petrilli, Claudio Geraldo Schon, Intrinsic uncertainty on ab-initio phase diagram and compound formation energy calculations: BCC Mo-Fe as a test case. Submetido para publicação.

[50] J. C. Slater. Physical Review, 51, 846-851, 1937.

[51] O. Krogh Andersen. Physical Review B, 8, 3060-3083, 1975.

[52] M. Schlüter, James R. Chelikowsky, Steven G. Louie e Marvin L. Cohen. Physical Review B, 12, 4200-4214, 1975.

[53] D. R. Hamann, Physical Review Letters, 42, 662-665, 1979.

[54] E. Wimmer, H. Krakauer, M. Weinert e A. J. Freeman. Physical Review B, 24, 864-875, 1981.

[55] Eugene Butkov, Física Matemática. Editora Guanabara Koogan S. A., 1968.

[56] M. Born e R. Oppenheimer. Zur Quantentheorie der Molekülen Annalen der Physik, 84,457-484,1927.

[57] G. Kresse e J. Furthmuller, Comp. Mater. Sci. 6, 15, 1996.

[58] L. A. Errico, M. Rentería e H. M. Petrilli, Physica B, 389, 37, 2007.

[59] Gehrard Inden e Wolfgang Pitch. Materials Science and Technology. Volume 5: Phase Transformations in Materials. Chapter 9: Atomic Ordering. pp. 499-552 Volume Editor: Peter Haasen. VCH Press, New York-1991.

[60] Cláudio Geraldo Schön. Tese de doutoramento: Thermodynamics of multicomponent systems with chemical and magnetic interactions. Universität Dortmund.Ph.D. Düsseldort/Dortmund 1998.

[61] Rezende Gomes dos Santos, "Transformações de fases em materiais metálicos", Editora da UNICAMP, 2006.

[62] R. Kikuchy. Journal of Chemical Physical, 6, 3, 1071-1080, 1974. 
[63] G. Inden, W. Pitsch, Atomic Ordering, vol.5 of Materials Science AlCrPhase and Technollogy, chapter 9, 497-552. VCH. Weinheim, Germany, 1991.

[64] Ryoichi Kikuchi. Journal of Phase Equilibria. 19, 412-422, 1998.

[65] Ryoichi Kikuchi e K. Masuda - Jindo. Computational Materials Science. 14, 295-310, 1999.

[66] C. Colinet, G. Inden e R. Kikuchi. Acta Metallurgica et Materialia, 41, 1109-1118, 1993.

[67] Ryoichi Kikuchi. Physical Review, 81, 988-1003, 1951.

[68] Ryoichi Kikuchi. The CVM Calculation of Phase Diagrams. in: Computer Modelling of Phase Diagrams, 49-65. AIME, 1986.

[69] J. A. Baker. Proceedings of the Royal Society in London, A216, 45-56, 1953.

[70] J. Hijmans e J. De Boer. Physica, 21, 471-484, 1955.

[71] J. Hijmans e J. De Boer. Physica, 21, 485-498, 1955.

[72] J. Hijmans e J. De Boer. Physica, 21, 499-516, 1955.

[73] J. Hijmans e J. De Boer. Physica, 22, 408-428, 1956.

[74] J. Hijmans e J. De Boer. Physica, 22, 429-442, 1956.

[75] T. Morita. Bulletin of the Americal Physical Society, 17, 79, 1972.

[76] T. Morita. Journal of Mathematical Physics, 13, 714, 1972.

[77] T. Morita. Journal of Mathematical Physics, 13, 1428, 1972.

[78] T. Morita. Journal of the Physical Society of Japan, 12, 753-755, 1957.

[79] T. Morita. Journal of the Physical Society of Japan, 12, 1060-1063, 1957.

[80] A. G. Schlijper. Physical Review B, 27, 6841-6848,1983.

[81] A. G. Schlijper. Jounal of Statistical Physics, 35, 285-301,1984.

[82] A. G. Schlijper. Jounal of Statistical Physics, 40, 1-27,1985.

[83] G. An. Journal of Statistical Physics, 52, 727-734, 1988. 
[84] C. G. Schön, G. Inden, Acta Materialia, 46, 4219-4231, 1998.

[85] I. Ohmuna, et al.. Acta Materialia, 46, 2083-2094,1998.

[86] P. G. G. Ormeño, H. M. Petrilli, C. G. Schön,. CALPHAD, 26, 573-582, 2002.

[87] Ryoichi Kikuchi. Physica A. 142, 321-341, 1987.

[88] Lawrence Hall Van Vlack. Princípios de ciência dos materiais. Edgar Blucher. São Paulo-1970.

[89] C. S. Wang, B. M. Klein e H. Krakauer. Physical Review Letters. 54, 1852-1855. 1985.

[90] P. Bagno, O. Jepsen e O. Gunnarson. Physical Review B, 40, 19972000, 1989.

[91] http://cst-www.nrl.navy.mil/lattice/index.html

[92] L. T. F. Eleno, C. G. Schön, CALPHAD, 27, 335-342, 2003.

[93] V. Blum e A. Zunger. Phys. Rev. B, 69, 020103(R), 2004.

[94] D. D. Koelling, B. N. Harmon, J. Phys. C Solid State Phys, 10, 3107$3114,1977$.

[95] A. H. MacDonald, W. E. Pickett, D. D. Koelling,J. Phys. C Solid State Phys, 13, 2675-2683, 1980.

[96] G. Kresse e D. Joubert, Phys. Rev. B, 59, 1758-1775, 1999.

[97] H. J. Monkhorst e J. D. Pack, Phys.Rev. B, 13, 5188-5192, 1976.

[98] M. Methfessel e A. T. Paxton, Phys. Rev. B, 40, 3616-3621, 1989.

[99] T. B. Massalski (Ed.), Binary Alloy Phase Diagrams, 2nd Edition, Vol. 1, ASM International, Metals Park-OH, 1990.

[100] J. A. Rayne e B. S. Chandrasekhar, Phys. Rev., 122, 1714-1716, 1961.

[101] J. M. Dickinson e P. E. Armstrong, J. Appl. Phys., 38, 602-606, 1967.

[102] R. Ferro, G. Borzone, G. Cacciamani e R. Raggio, Thermochimica Acta, 347, 103-122, 2000.

[103] C. G. Schön e G. Inden, Journal de Chimie Physique, 94, 1143-51, 1997. 
[104] C. T. Liu, J. Stringer, J.N. Mundy, L.L. Horton e P. Angelini, Intermetallics, 5, 579-96, 1997.

[105] M.V. Nathal, C.T. Liu, Intermetallics, 3, 77-81, 1995.

[106] S. C. Deevi, V. K. Sikka e C. T. Liu, Progr. Mat. Sci., 42, 177-92, 1997.

[107] A. Pasturel, C. Colinet, A. T. Paxton e M. van Schilfgaarde. Journal of Physics: Condensed Matter, 41, 649-684, 1992.

[108] Ortrud Kubaschewski. Iron-Binary Phase Diagrams. Springer-Verlag Berlin-1982.

[109] Pablo Guillermo Gonzales-Ormeño, Helena Maria Petrilli e Cláudio Geraldo Schön, Scripta Materialia, 54, 7, 1271-1276, 2006.

[110] Ney Sodré, Pablo Guillermo Gonzales-Ormeño, Helena Maria Petrilli e Cláudio Geraldo Schön, Calphad, 33, 576-583, 2009.

[111] O. Kubaschewski e W. A. Dench. Acta Metallurgica, 3, 339-346, 1955.

[112] S. V. Radcliffe, B. L. Averbach e M. Cohen. Acta Metallurgica, 9, 169$176,1961$.

[113] T. Helander e O. Tolochko, J. Phase, 20(1), 57-60, 1999.

[114] Yu Liang, Cuiping Guo, Changrong Li e Zhenmin Du, Journal of Alloys and Compounds, 460, 314-319, 2008.

[115] P. G. G. Ormeño, N. Sodre, H. M. Petrilli, C. G. Schön, CALPHAD XL, may, 22th-27th, RJ, 2011.

[116] H. Okamoto, Journal of Phase Equilibria and Diffusion, 29, N.01, 2008.

[117] B. B. Cao e K. H. Kuo, Journal of Alloys and Compounds, 458, 238247, 2008.

[118] P. G. Gonzales-Ormeño, H. M. Petrilli, C. G. Schön, Scripta Materialli. 53, 751-756, 2005.

[119] Gehrard Inden e Wolfgang Pitch. Materials Science and Technology. Volume 5: Phase Transformations in materials. Chapter 9: Atomic Ordering. 499-552. VCH Press, New York - 1991.

[120] Rhines Frederick Nims. Phase diagrams in metallurgy: Their development and application. New York, Mc. Graw-Hill-1956. 
[121] P. Novák. Calculation of hyperfine field in WIEN2K-Technical report, http://www.wien2k.at, 2006.

[122] P. Novak. Calculation of spin-orbit coupling, http://www.wien2k.at

[123] G.Bonny, D.Terentyev e L. Malerba, Scripta Materialia, 59, 1193-1196, 2008. 